Supporting Information

\title{
Experimental and Computational Probes of a Self-Assembled Capsule
}

\author{
Tetsuo Iwasawa, Dariush Ajami, and Julius Rebek, Jr.*
}

The Skaggs Institute for Chemical Biology and the Department of Chemistry, The Scripps Research Institute

MB-26, 10550 North Torrey Pines Road, La Jolla, California 92037

\section{Contents}

(1) General (page 1S)

(2) Materials (page 1S)

(3) Complete list of author for Guassian, Inc., Pittsburgh, PA, 2002 (page 2S)

(4) Preparation of biaryls in Figure 1b. (page 2S-6S)

(5) Competitive encapsulation studies. (page 6S)

(6) Reference (page 6S - 7S)

(7) ${ }^{1} \mathrm{H}$ and ${ }^{13} \mathrm{C}$ NMR spectra of biaryls 2a-g (page 8S - 21S), 3b-g (page 22S 34S) 4 (page 35S - 36S), 6d (page 37S - 38S), 7a-c (page 39S-44S), and all ${ }^{1} \mathrm{H}$ NMR spectra of encapsulated biaryls (page $45 S$ - 52S) and competitive encapsulation studies (page 53S - 57S).

(1) General NMR spectra were recorded on a Bruker DRX-600 spectrometer. Proton $\left({ }^{1} \mathrm{H}\right)$ chemical shifts, reported in parts per million (ppm), were indirectly referenced to external tetramethylsilane employing resonances due to trace monoprotio-solvent as an internal reference. Deuterated NMR solvents were obtained from Cambridge Isotope Laboratories, Inc., and used without further purification.

(2) Materials Compounds 3a, 6a - 6c, and 5 were purchased from Aldrich Chemical Company, and were used as received. The compound $\mathbf{6 e}$ was purchased from Acros Organics, and were used as received. The starting materials for other biaryls, 3-bromopyridine, 2-bromopyridine, 2-bromo-5-methylpyridine, 4-bromotoluene, 5-bromopyrimidine, p-tolylboronic acid, 4-ethylphenylboronic acid, 
4-n-propylphenylboronic acid, and 4-n-butylphenylboronic acid, were purchased from Aldrich Chemical Company., and were used without further purification. The 5-bromo-2-methylpyridine was purchased from Apollo Scientific Ltd., and was used without further purification. The reagents for cross-coupling reactions, $\mathrm{Pd}(\mathrm{OAc})_{2}, \mathrm{~K}_{3} \mathrm{PO}_{4}$, $\mathrm{K}_{2} \mathrm{CO}_{3}$, and tetra- $n$-butylammonium bromide were purchased from Aldrich Chemical Company. The catalyst $\mathrm{Pd}(\mathrm{OAc})_{2}\left(\mathrm{Cy}_{2} \mathrm{NH}\right)_{2}$ was prepared according to the literature. ${ }^{1}$ The cylindrical cavitand $\mathbf{1}$ was prepared according to the literature. ${ }^{2}$

(3) Complete list of author for Guassian, Inc., Pittsburgh, PA, 2002: Frisch, M. J.; Trucks, G. W.; Schlegel, H. B.; Scuseria, G. E.; Robb, M. A.; Cheeseman, J. R.; Montgomery, Jr., J. A.; Vreven, T.; Kudin, K. N.; Burant, J. C.; Millam, J. M.; Iyengar, S. S.; Tomasi, J.; Barone, V.; Mennucci, B.; Cossi, M.; Scalmani, G.; Rega, N.; Petersson, G. A.; Nakatsuji, H.; Hada, M.; Ehara, M.; Toyota, K.; Fukuda, R.; Hasegawa, J.; Ishida, M.; Nakajima, T.; Honda, Y.; Kitao, O.; Nakai, H.; Klene, M.; Li, X.; Knox, J. E.; Hratchian, H. P.; Cross, J. B.; Bakken, V.; Adamo, C.; Jaramillo, J.; Gomperts, R.; Stratmann, R. E.; Yazyev, O.; Austin, A. J.; Cammi, R.; Pomelli, C.; Ochterski, J. W.; Ayala, P. Y.; Morokuma, K.; Voth, G. A.; Salvador, P.; Dannenberg, J. J.; Zakrzewski, V. G.; Dapprich, S.; Daniels, A. D.; Strain, M. C.; Farkas, O.; Malick, D. K.; Rabuck, A. D.; Raghavachari, K.; Foresman, J. B.; Ortiz, J. V.; Cui, Q.; Baboul, A. G.; Clifford, S.; Cioslowski, J.; Stefanov, B. B.; Liu, G.; Liashenko, A.; Piskorz, P.; Komaromi, I.; Martin, R. L.; Fox, D. J.; Keith, T.; Al-Laham, M. A.; Peng, C. Y.; Nanayakkara, A.; Challacombe, M.; Gill, P. M. W.; Johnson, B.; Chen, W.; Wong, M. W.; Gonzalez, C.; and Pople, J. A.; Gaussian, Inc., Wallingford CT, 2004.

(4) Preparation of biaryls in Figure $1 \mathbf{b}$. The biaryls $2 a-2 g, 3 b-3 g, 6 d$, and $7 a-7 c$ were prepared according to the Suzuki-Miyaura cross-coupling under aerobic conditions. ${ }^{1}$ The synthesis of biaryl $\mathbf{4}$ was referred to the literature. ${ }^{3}$ Typical procedures about $\mathbf{2 f}$ and $\mathbf{4}$ were described below, as well as data of other biaryls. The ${ }^{1} \mathrm{H}$ NMR and ${ }^{13} \mathrm{C}$ NMR spectra of $\mathbf{2 a}-\mathbf{2 g}, \mathbf{3 b}-\mathbf{3 g}, \mathbf{4}, \mathbf{6 d}$, and $\mathbf{7 a}-\mathbf{7} \mathbf{c}$ are also attached in from page $8 \mathrm{~S}-44 \mathrm{~S}$.

2f: In the air, to a $50 \mathrm{~mL}$ one neck flask $\mathrm{K}_{3} \mathrm{PO}_{4}(850 \mathrm{mg}, 4 \mathrm{mmol}), \mathrm{Pd}(\mathrm{OAc})_{2}\left(\mathrm{Cy}{ }_{2} \mathrm{NH}\right)_{2}(23$ mg, $0.04 \mathrm{mmol}$ ), 5-bromo-2-methylpyridine (344 mg, $2 \mathrm{mmol}$ ), 4-n-propylboronic acid (394 mg, $2.4 \mathrm{mmol}$ ), and EtOH (6 mL) were added. After stirring at ambient temperature 
for overnight, EtOAc (50 mL) was added, and the mixture was washed with water and brine, and dried over $\mathrm{Na}_{2} \mathrm{SO}_{4}$. Evaporation of the solvent provided the crude product, which was purified by column chromatography to afford the desired products in 50\% yield (210 mg) as pale yellow crystals. ${ }^{1} \mathrm{H}$ NMR $\left(600 \mathrm{MHz}, \mathrm{C}_{6} \mathrm{D}_{6}\right) \delta 8.91(\mathrm{~d}, J=2.4 \mathrm{~Hz}, 1 \mathrm{H}), 7.40$ (dd, $J=2.4 \mathrm{~Hz}, 7.8 \mathrm{~Hz}, 1 \mathrm{H}$ ), 7.30 (d, $J=8.4 \mathrm{~Hz}, 2 \mathrm{H}), 7.04$ (d, $J=8.4 \mathrm{~Hz}, 2 \mathrm{H}), 6.74$ (d, $J=$ $7.8 \mathrm{~Hz}, 1 \mathrm{H}), 2.47$ (s, 3H), 2.43 (t, $J=7.2 \mathrm{~Hz}, 2 \mathrm{H}), 1.52$ (dt, $J=7.2 \mathrm{~Hz}, 7.2 \mathrm{~Hz}, 2 \mathrm{H}$ ), 0.849 (t, $J=7.2 \mathrm{~Hz}, 3 \mathrm{H}) .{ }^{13} \mathrm{C}$ NMR $\left(150 \mathrm{MHz}, \mathrm{C}_{6} \mathrm{D}_{6}\right) \delta 157.3,148.0,142.2,136.0,134.1,133.8$, 129.4, 127.1, 122.9, 37.9, 24.8, 24.2, 13.9. HRMS (ESI, $\mathrm{MH}^{+}$): Calcd. For $\mathrm{C}_{15} \mathrm{H}_{18} \mathrm{~N}$ : 212.1434. Found: 212.1440.

$4^{3}$ : Under a nitrogen atmosphere, a mixture of $\mathrm{K}_{2} \mathrm{CO}_{3}(1.10 \mathrm{~g}, 8 \mathrm{mmol}), \mathrm{Pd}(\mathrm{OAc})_{2}(90 \mathrm{mg}$, $0.4 \mathrm{mmol})$, tetra- $n$-butylammonium bromide $(1.29 \mathrm{~g}, 4 \mathrm{mmol})$ and 5-bromo-2-methylpyridine (1.37 g, $8 \mathrm{mmol})$ in a mix of DMF $(0.9 \mathrm{~mL}) / \mathrm{H}_{2} \mathrm{O}(0.35 \mathrm{~mL})$ was stirred for 2 minutes at $115{ }^{\circ} \mathrm{C}$. Isopropanol (480 mg, $8 \mathrm{mmol}$ ) was added. After stirring for $48 \mathrm{~h}$, the reaction mixture was allowed to cool to ambient temperature. To the mixture was water and ether, and the organic phase was washed with water and brine, and dried over $\mathrm{Na}_{2} \mathrm{SO}_{4}$. Evaporation of the solvent provided the crude product, which was purified by column chromatography to afford the desired products in 51\% yield (377 $\mathrm{mg}$ ) as white crystals. ${ }^{1} \mathrm{H}$ NMR $\left(600 \mathrm{MHz}, \mathrm{C}_{6} \mathrm{D}_{6}\right) \delta 8.70(\mathrm{~d}, J=2.4 \mathrm{~Hz}, 2 \mathrm{H}), 7.18$ (dd, $J=2.4$ $\mathrm{Hz}, 7.8 \mathrm{~Hz}, 2 \mathrm{H}), 6.66$ (d, $J=7.8 \mathrm{~Hz}, 2 \mathrm{H}), 2.44$ (s, 6H). ${ }^{13} \mathrm{C}$ NMR (150 MHz, $\left.{ }_{6} \mathrm{D}_{6}\right)$ $\delta$ 157.9, 147.9, 134.0, 130.8, 123.0, 24.2. HRMS (ESI, $\mathrm{MH}^{+}$): Calcd. For $\mathrm{C}_{12} \mathrm{H}_{13} \mathrm{~N}_{2}$ : 185.1073. Found: 185.1067.

2a ${ }^{5}:{ }^{1} \mathrm{H}$ NMR (600 MHz, $\left.\mathrm{C}_{6} \mathrm{D}_{6}\right) \delta 8.97(\mathrm{~d}, J=1.2 \mathrm{~Hz}, 1 \mathrm{H}), 8.52(\mathrm{~d}, J=3.6 \mathrm{~Hz}, 1 \mathrm{H}), 7.39-$ 7.37 (m, 1H), 7.21 (d, $J=8.4 \mathrm{~Hz}, 2 \mathrm{H}), 6.96$ (d, $J=8.4 \mathrm{~Hz}, 2 \mathrm{H}), 6.78-6.77$ (m, 1H), 2.10 (s, 3H). ${ }^{13} \mathrm{C}$ NMR (150 MHz, $\left.{ }_{6} \mathrm{D}_{6}\right) \delta 148.8,148.7,137.7,136.6,135.5,133.6,129.9,127.2$, 123.4, 21.0. HRMS (ESI, $\mathrm{MH}^{+}$): Calcd. For $\mathrm{C}_{12} \mathrm{H}_{11} \mathrm{~N}$ : 170.0964. Found: 170.0970 .

2b: ${ }^{1} \mathrm{H}$ NMR (600 MHz, $\mathrm{C}_{6} \mathrm{D}_{6}$ ) $\delta 8.89$ (d, $J=2.4 \mathrm{~Hz}, 1 \mathrm{H}$ ), 7.39 (dd, $J=2.4 \mathrm{~Hz}, 7.8 \mathrm{~Hz}, 1 \mathrm{H}$ ), 7.25 (d, $J=8.4 \mathrm{~Hz}, 2 \mathrm{H}$ ), 6.99 (d, $J=8.4 \mathrm{~Hz}, 2 \mathrm{H}), 6.73$ (d, $J=7.8 \mathrm{~Hz}, 1 \mathrm{H}$ ), 2.48 (s, 3H), 2.11 (s, 3H). ${ }^{13} \mathrm{C}$ NMR (150 MHz, $\left.\mathrm{C}_{6} \mathrm{D}_{6}\right) \delta 157.3,148.0,137.4,135.7,134.1,133.8$, 129.9, 127.1, 122.9, 24.2, 21.0. HRMS (ESI, $\mathrm{MH}^{+}$): Calcd. For $\mathrm{C}_{13} \mathrm{H}_{13} \mathrm{~N}$ : 184.1121. Found: 184.1123. 
2c: ${ }^{1} \mathrm{H}$ NMR (600 MHz, $\left.\mathrm{C}_{6} \mathrm{D}_{6}\right) \delta 8.98(\mathrm{~d}, J=1.8 \mathrm{~Hz}, 1 \mathrm{H}), 8.52$ (dd, $\left.J=1.8 \mathrm{~Hz}, 4.8 \mathrm{~Hz}, 1 \mathrm{H}\right)$, $7.41-7.39$ (m, 1H), 7.25 (d, $J=7.8 \mathrm{~Hz}, 2 \mathrm{H}$ ), 7.02 (d, $J=7.8 \mathrm{~Hz}, 2 \mathrm{H}$ ), 6.78 (dd, $J=4.8 \mathrm{~Hz}$, $8.4 \mathrm{~Hz}, 1 \mathrm{H}), 2.44$ (q, $J=7.8 \mathrm{~Hz}, 2 \mathrm{H}), 1.08(\mathrm{t}, J=7.8 \mathrm{~Hz}, 3 \mathrm{H}) .{ }^{13} \mathrm{C}$ NMR (150 MHz, $\left.\mathrm{C}_{6} \mathrm{D}_{6}\right) \delta 148.8,148.7,144.1,136.6,135.8,133.7,128.8,127.4,123.4,28.8,15.7 . \quad$ HRMS (ESI, $\mathrm{MH}^{+}$): Calcd. For $\mathrm{C}_{13} \mathrm{H}_{14} \mathrm{~N}$ : 184.1121. Found: 184.1128.

2d: ${ }^{1} \mathrm{H}$ NMR (600 MHz, C $6 \mathrm{D}_{6}$ ) $\delta 8.91$ (s, 1H), 7.40 (dd, $J=2.4 \mathrm{~Hz}, 7.8 \mathrm{~Hz}, 1 \mathrm{H}$ ), 7.29 (d, $J$ $=8.4 \mathrm{~Hz}, 2 \mathrm{H}), 7.03$ (d, $J=8.4 \mathrm{~Hz}, 2 \mathrm{H}), 6.74(\mathrm{~d}, J=7.8 \mathrm{~Hz}, 1 \mathrm{H}), 2.47$ (s, 3H), 2.45 (q, $J=$ $7.8 \mathrm{~Hz}, 2 \mathrm{H}), 1.09$ (t, $J=7.8 \mathrm{~Hz}, 3 \mathrm{H}) .{ }^{13} \mathrm{C} \mathrm{NMR}\left(150 \mathrm{MHz}, \mathrm{C}_{6} \mathrm{D}_{6}\right) \delta 157.3,148.0,143.8$, 135.9, 134.1, 133.8, 128.7, 127.2, 122.9, 28.8, 24.2, 15.8. HRMS (ESI, $\mathrm{MH}^{+}$): Calcd. For $\mathrm{C}_{14} \mathrm{H}_{16} \mathrm{~N}:$ 198.1277. Found: 198.1280 .

2e: ${ }^{1} \mathrm{H}$ NMR (600 MHz, $\left.\mathrm{C}_{6} \mathrm{D}_{6}\right) \delta 8.99(\mathrm{~d}, J=2.4 \mathrm{~Hz}, 1 \mathrm{H}), 8.52$ (dd, $J=2.4 \mathrm{~Hz}, 4.8 \mathrm{~Hz}, 1 \mathrm{H}$ ), $7.41-7.39$ (m, 1H), 7.25 (d, $J=8.4 \mathrm{~Hz}, 2 \mathrm{H}$ ), 7.01 (d, $J=8.4 \mathrm{~Hz}, 2 \mathrm{H}), 2.42$ (t, $J=7.8 \mathrm{~Hz}$, 2H), 1.52 (dt, $J=7.8 \mathrm{~Hz}, 7.8 \mathrm{~Hz}, 2 \mathrm{H}), 0.84$ (t, $J=7.8 \mathrm{~Hz}, 3 \mathrm{H}) .{ }^{13} \mathrm{C}$ NMR $(150 \mathrm{MHz}$, $\left.\mathrm{C}_{6} \mathrm{D}_{6}\right) \delta 148.8,148.7,142.5,136.6,135.8,133.7,129.7,127.3,123.4,37.9,24.8,13.9$. HRMS (ESI, $\mathrm{MH}^{+}$): Calcd. For $\mathrm{C}_{14} \mathrm{H}_{16} \mathrm{~N}$ : 198.1277. Found: 198.1284.

$2 \mathbf{g}^{6}:{ }^{1} \mathrm{H}$ NMR (600 MHz, $\left.\mathrm{C}_{6} \mathrm{D}_{6}\right) \delta 9.00$ (d, $\left.J=2.4 \mathrm{~Hz}, 1 \mathrm{H}\right), 8.53-8.52$ (m, 1H), $7.42-7.40$ (m, 1H), 7.26 (d, $J=8.4 \mathrm{~Hz}, 2 \mathrm{H}), 7.03$ (d, $J=8.4 \mathrm{~Hz}, 2 \mathrm{H}), 6.79-6.77$ (m, 1H), 2.46 (t, $J=$ $7.8 \mathrm{~Hz}, 2 \mathrm{H}), 1.49$ (dd, $J=7.8 \mathrm{~Hz}, 7.8 \mathrm{~Hz}, 2 \mathrm{H}), 1.24$ (dt, $J=7.2 \mathrm{~Hz}, 7.8 \mathrm{~Hz}, 2 \mathrm{H}), 0.86$ (t, $J=$ $7.2 \mathrm{~Hz}, 3 \mathrm{H}) .{ }^{13} \mathrm{C}$ NMR $\left(150 \mathrm{MHz}, \mathrm{C}_{6} \mathrm{D}_{6}\right) \delta 148.8,148.7,142.8,136.6,135.8,133.7,129.4$, 127.3, 123.4, 35.6, 33.9, 22.6, 14.1. HRMS (ESI, $\mathrm{MH}^{+}$): Calcd. For $\mathrm{C}_{15} \mathrm{H}_{18} \mathrm{~N}$ : 212.1434. Found: 212.1434 .

$3 \mathbf{b}^{7}:{ }^{1} \mathrm{H}$ NMR (600 MHz, $\left.{ }_{6} \mathrm{D}_{6}\right) \delta 8.49$ (d, $\left.J=2.4 \mathrm{~Hz}, 1 \mathrm{H}\right), 8.15$ (d, $\left.J=7.8 \mathrm{~Hz}, 2 \mathrm{H}\right), 7.35$ (d, $J=8.4 \mathrm{~Hz}, 1 \mathrm{H}$ ), 7.11 (d, $J=7.8 \mathrm{~Hz}, 2 \mathrm{H}), 6.99$ (dd, $J=2.4 \mathrm{~Hz}, 8.4 \mathrm{~Hz}, 1 \mathrm{H}$ ), 2.13 (s, 3H), 1.85 (s, 3H). ${ }^{13} \mathrm{C}$ NMR (150 MHz, $\left.\mathrm{C}_{6} \mathrm{D}_{6}\right) \delta 155.0,150.5,138.5,137.3,136.8,131.0$, 129.7, 127.0, 119.2, 21.2, 17.8. MS (EI, $\mathrm{m} / \mathrm{z}): 183\left(\mathrm{M}^{+}\right)$.

3c: ${ }^{1} \mathrm{H}$ NMR (600 MHz, $\left.\mathrm{C}_{6} \mathrm{D}_{6}\right) \delta 8.55-8.53(\mathrm{~m}, 1 \mathrm{H}), 8.02(\mathrm{~d}, J=7.8 \mathrm{~Hz}, 2 \mathrm{H}), 7.36(\mathrm{~d}, J=$ $7.8 \mathrm{~Hz}, 1 \mathrm{H}), 7.24-7.21(\mathrm{~m}, 1 \mathrm{H}), 7.12$ (d, $J=7.8 \mathrm{~Hz}, 2 \mathrm{H}), 6.75-6.73$ (m, 1H), 2.55 (q, $J=$ 
$7.2 \mathrm{~Hz}, 1 \mathrm{H}), 1.20$ (t, $J=7.2 \mathrm{~Hz}, 3 \mathrm{H}) .{ }^{13} \mathrm{C} \mathrm{NMR}\left(150 \mathrm{MHz}, \mathrm{C}_{6} \mathrm{D}_{6}\right) \delta 157.6,149.9,145.3$, 137.5, 136.2, 128.5, 127.3, 121.7, 119.8, 28.9, 15.6. HRMS (ESI, $\mathrm{MH}^{+}$): Calcd. For $\mathrm{C}_{13} \mathrm{H}_{14} \mathrm{~N}$ : 184.1121. Found: 184.1128.

3d: ${ }^{1} \mathrm{H}$ NMR (600 MHz, $\left.\mathrm{C}_{6} \mathrm{D}_{6}\right) \delta 8.49-8.48(\mathrm{~m}, 1 \mathrm{H}), 8.17$ (d, $\left.J=7.8 \mathrm{~Hz}, 2 \mathrm{H}\right), 7.36$ (d, $J=$ $7.8 \mathrm{~Hz}, 2 \mathrm{H}$ ), $7.16-7.14$ (m, 1H), 6.99 (dd, $J=2.4 \mathrm{~Hz}, 7.8 \mathrm{~Hz}, 1 \mathrm{H}$ ), 2.47 (q, $J=7.8 \mathrm{~Hz}$, 2H), 1.85 (s, 3H), 1.09 (t, $J=7.8 \mathrm{~Hz}, 3 \mathrm{H}) .{ }^{13} \mathrm{C}$ NMR (150 MHz, $\left.\mathrm{C}_{6} \mathrm{D}_{6}\right) \delta$ 155.0, 150.4, 144.9, 137.5, 136.9, 131.1, 128.5, 127.1, 119.4, 28.9, 17.8, 15.7. HRMS (ESI, $\mathrm{MH}^{+}$): Calcd. For $\mathrm{C}_{14} \mathrm{H}_{16} \mathrm{~N}$ : 198.1277 . Found: 198.1283.

3e: ${ }^{1} \mathrm{H}$ NMR (600 MHz, $\left.\mathrm{C}_{6} \mathrm{D}_{6}\right) \delta 8.60-8.58(\mathrm{~m}, 1 \mathrm{H}), 8.14$ (d, $\left.J=8.4 \mathrm{~Hz}, 2 \mathrm{H}\right), 7.38$ (d, $J=$ $7.8 \mathrm{~Hz}, 1 \mathrm{H}$ ), $7.14-7.11$ (m, 3H), 6.65 (m, 1H), 2.44 (t, $J=7.2 \mathrm{~Hz}, 2 \mathrm{H}), 1.51$ (dt, $J=7.2$ $\mathrm{Hz}, 7.8 \mathrm{~Hz}, 2 \mathrm{H}), 0.83(\mathrm{t}, J=7.8 \mathrm{~Hz}, 3 \mathrm{H}) .{ }^{13} \mathrm{C} \mathrm{NMR}\left(150 \mathrm{MHz}, \mathrm{C}_{6} \mathrm{D}_{6}\right) \delta 157.6,150.0$, 143.7, 137.5, 136.3, 129.1, 127.2, 121.7, 119.8, 38.0, 24.7, 13.9. HRMS (ESI, $\mathrm{MH}^{+}$): Calcd. For $\mathrm{C}_{14} \mathrm{H}_{16} \mathrm{~N}$ : 198.1277 . Found: 198.1279 .

3f: ${ }^{1} \mathrm{H}$ NMR (600 MHz, $\left.\mathrm{C}_{6} \mathrm{D}_{6}\right) \delta 8.51-8.49(\mathrm{~m}, 1 \mathrm{H}), 8.19(\mathrm{~d}, J=8.4 \mathrm{~Hz}, 2 \mathrm{H}), 7.37$ (d, $J=$ $8.4 \mathrm{~Hz}, 1 \mathrm{H}$ ), $7.16-7.14$ (m, 3H), $7.00-6.97$ (m, 1H), 2.45 (t, J= 7.8 Hz, 2H), 1.85 (s, 3H), 1.53 (dt, $J=7.8 \mathrm{~Hz}, 7.2 \mathrm{~Hz}, 2 \mathrm{H}), 0.85$ (t, $J=7.2 \mathrm{~Hz}, 3 \mathrm{H}) .{ }^{13} \mathrm{C}$ NMR $\left(150 \mathrm{MHz}, \mathrm{C}_{6} \mathrm{D}_{6}\right.$ ) $\delta 155.1,150.5,143.3,137.6,136.9,131.1,129.1,127.0,119.3,38.0,24.8,17.8,13.9$. HRMS (ESI, $\mathrm{MH}^{+}$): Calcd. For $\mathrm{C}_{15} \mathrm{H}_{18} \mathrm{~N}$ : 212.1434. Found: 212.1436.

$3 \mathbf{g}^{8}:{ }^{1} \mathrm{H}$ NMR (600 MHz, $\left.\mathrm{C}_{6} \mathrm{D}_{6}\right) \delta 8.60-8.59(\mathrm{~m}, 1 \mathrm{H}), 8.15$ (d, $\left.J=7.8 \mathrm{~Hz}, 2 \mathrm{H}\right), 7.38-7.37$ (m, 1H), 7.13 (d, $J=7.8 \mathrm{~Hz}, 2 \mathrm{H}), 7.12-7.10$ (m, 1H), 6.64 (dd, $J=4.8 \mathrm{~Hz}, 7.2 \mathrm{~Hz}, 1 \mathrm{H}$ ), 2.48 (t, $J=7.8 \mathrm{~Hz}, 2 \mathrm{H}$ ), 1.49 (dd, $J=7.8 \mathrm{~Hz}, 7.2 \mathrm{~Hz}, 2 \mathrm{H}$ ), 1.23 (dt, $J=7.8 \mathrm{~Hz}, 7.2 \mathrm{~Hz}, 2 \mathrm{H}$ ), 0.85 (t, $J=7.8 \mathrm{~Hz}, 3 \mathrm{H}$ ). HRMS (ESI, $\mathrm{MH}^{+}$): Calcd. For $\mathrm{C}_{15} \mathrm{H}_{18} \mathrm{~N}$ : 212.1434. Found: 212.1439.

6d $\mathbf{d}^{9}:{ }^{1} \mathrm{H}$ NMR (600 MHz, $\mathrm{CDCl}_{3}$ ) $\delta 7.49$ (d, $\left.J=8.4 \mathrm{~Hz}, 2 \mathrm{H}\right), 7.47$ (d, $J=7.8 \mathrm{~Hz}, 2 \mathrm{H}$ ), 7.10 (d, $J=7.8 \mathrm{~Hz}, 2 \mathrm{H}), 7.07$ (d, $J=8.4 \mathrm{~Hz}, 2 \mathrm{H}), 2.49$ (q, $J=7.8 \mathrm{~Hz}, 2 \mathrm{H}), 2.16$ (s, 3H), 1.12 (t, $J=7.8 \mathrm{~Hz}, 3 \mathrm{H}) .{ }^{13} \mathrm{C}$ NMR $\left(150 \mathrm{MHz}, \mathrm{C}_{6} \mathrm{D}_{6}\right) \delta 143.1,139.1,138.9,136.6,129.8,128.6$, 127.3, 127.2, 28.8, 21.1, 15.9. MS (EI, $m / z): 196\left(\mathrm{M}^{+}\right)$. 
7a ${ }^{10}:{ }^{1} \mathrm{H}$ NMR (600 MHz, $\mathrm{CDCl}_{3}$ ) $\delta 9.18$ (s, $\left.1 \mathrm{H}\right), 8.93$ (s, 2H), 7.48 (d, $J=8.4 \mathrm{~Hz}, 2 \mathrm{H}$ ), 7.31 (d, $J=8.4 \mathrm{~Hz}, 2 \mathrm{H}), 2.42$ (s, 3H). ${ }^{13} \mathrm{C}$ NMR (150 MHz, $\left.\mathrm{C}_{6} \mathrm{D}_{6}\right) \delta 157.1,154.7,139.1$, 134.2, 131.3, 130.1, 126.8, 21.2. MS (EI, $\mathrm{m} / \mathrm{z}): 170\left(\mathrm{M}^{+}\right)$.

7b: ${ }^{1} \mathrm{H}$ NMR (600 MHz, $\mathrm{CDCl}_{3}$ ) $\delta 9.27$ (s, 1H), 8.69 (s, 2H), 7.02 (d, $J=8.4 \mathrm{~Hz}, 2 \mathrm{H}$ ), 6.94 (d, $J=8.4 \mathrm{~Hz}, 2 \mathrm{H}), 2.40$ (q, $J=7.2 \mathrm{~Hz}, 2 \mathrm{H}), 1.06$ (t, $J=7.2 \mathrm{~Hz}, 3 \mathrm{H}) .{ }^{13} \mathrm{C}$ NMR $(150 \mathrm{MHz}$, $\left.\mathrm{C}_{6} \mathrm{D}_{6}\right) \delta 157.9,154.7,144.9,134.2,132.2,128.9,127.1,28.8,15.6 . \quad$ HRMS (ESI, $\mathrm{MH}^{+}$): Calcd. For $\mathrm{C}_{12} \mathrm{H}_{13} \mathrm{~N}_{2}$ : 185.1073. Found: 185.1079.

7c: ${ }^{1} \mathrm{H}$ NMR (600 MHz, $\left.\mathrm{C}_{6} \mathrm{D}_{6}\right) \delta 9.27$ (s, 1H), 8.69 (s, 2H), 7.03 (d, $\left.J=7.8 \mathrm{~Hz}, 2 \mathrm{H}\right), 6.95$ (d, $J=7.8 \mathrm{~Hz}, 2 \mathrm{H}$ ), 2.38 (t, $J=7.2 \mathrm{~Hz}, 2 \mathrm{H}), 1.48$ (dd, $J=7.8 \mathrm{~Hz}, 7.2 \mathrm{~Hz}, 2 \mathrm{H}), 0.83$ (t, $J=7.8$ $\mathrm{Hz}, 3 \mathrm{H}) .{ }^{13} \mathrm{C}$ NMR (150 MHz, $\left.\mathrm{C}_{6} \mathrm{D}_{6}\right) \delta 158.0,154.7,143.4,134.1,132.2,129.5,127.0$, 37.8, 24.7, 13.8. HRMS (ESI, $\mathrm{MH}^{+}$): Calcd. For $\mathrm{C}_{13} \mathrm{H}_{15} \mathrm{~N}_{2}$ : 199.1230. Found: 199.1226.

\section{(5) Competitive encapsulation studies}

In the competitive encapsulations in Table 1 and Table 2, the concentration of 1 was $4 \mathrm{mM}$, and that of each guest was performed in $50 \mathrm{mM}$. The mixture of the host and guests was warmed and sonicated to dissolve the host-guest complexes, and placed at ambient temperature for at least $30 \mathrm{~min}$. The ratios were based on the peaks of alkyl side-chains located at the upfield of ${ }^{1} \mathrm{H}$ NMR spectra, and determined on the integral values and/or weighing papers of the magnified peaks. All the ${ }^{1} \mathrm{H}$ NMR spectra of encapsulated guests and the pairwise competition of Table 1 and Table 2 are shown in from page 45S to 57S.

\section{(6) Reference}

1) Tao, B.; Boykin, D. W. J. Org. Chem. 2004, 69, 4330 - 4335.

2) Hayashida, O.; Sebo, L.; Rebek, J., Jr. J. Org. Chem. 2002, 67, 8291 - 8298.

3) Hassan, J.; Penalva, V.; Lavenot, L.; Gozzi, C.; Lemaire, M. Tetrahedron 1998, 54, $13793-13804$.

4) Cherioux, F.; Attias, A.-J.; Maillotte, H. Advanced Functional Materials 2002, 12, 203 208.

5) Inada, K.; Miyaura, N. Tetrahedron 2000, 56, 8661 - 8664.

6) Barder, T. E.; Walker, S. D.; Martinelli, J. R.; Buchwald, S. L. J. Am. Chem. Soc. 2005, 
127, $4685-4696$.

7) Jung, S.; Kang, Y.; Kim, H.-S.; Kim, Y.-H.; Lee, C.-L., Kim, J.-J.; Lee, S.-K.; Kwon, S.-K. Eur. J. Inorg. Chem. 2004, 3415 - 3423.

8) Chia, W.-L.; Shen, S. -W.; Lin, H.-C. Tetrahedron Lett. 2001, 42, 2177 - 2179.

9) Cho, C.-H.; Yun, H.-S.; Park, K. J. Org. Chem. 2003, 68, 3017 -3025.

10) Kano, S.; Yuasa, Y.; Shibuya, S.; Hibino, S. Heterocycles 1982, 19, 1079 - 1082.

(7) ${ }^{1} \mathrm{H}$ and ${ }^{13} \mathrm{C}$ NMR spectra of biaryls 2a-g (page 8S - 21S), 3b-g (page 22S - 34S) 4 (page 35S - 36S), 6d (page 37S - 38S), 7a-c (page 39S - 44S), and all ${ }^{1} \mathrm{H}$ NMR spectra of encapsulated biaryls (page 45S - 52S) and competitive encapsulation studies (page 53S - 57S). 


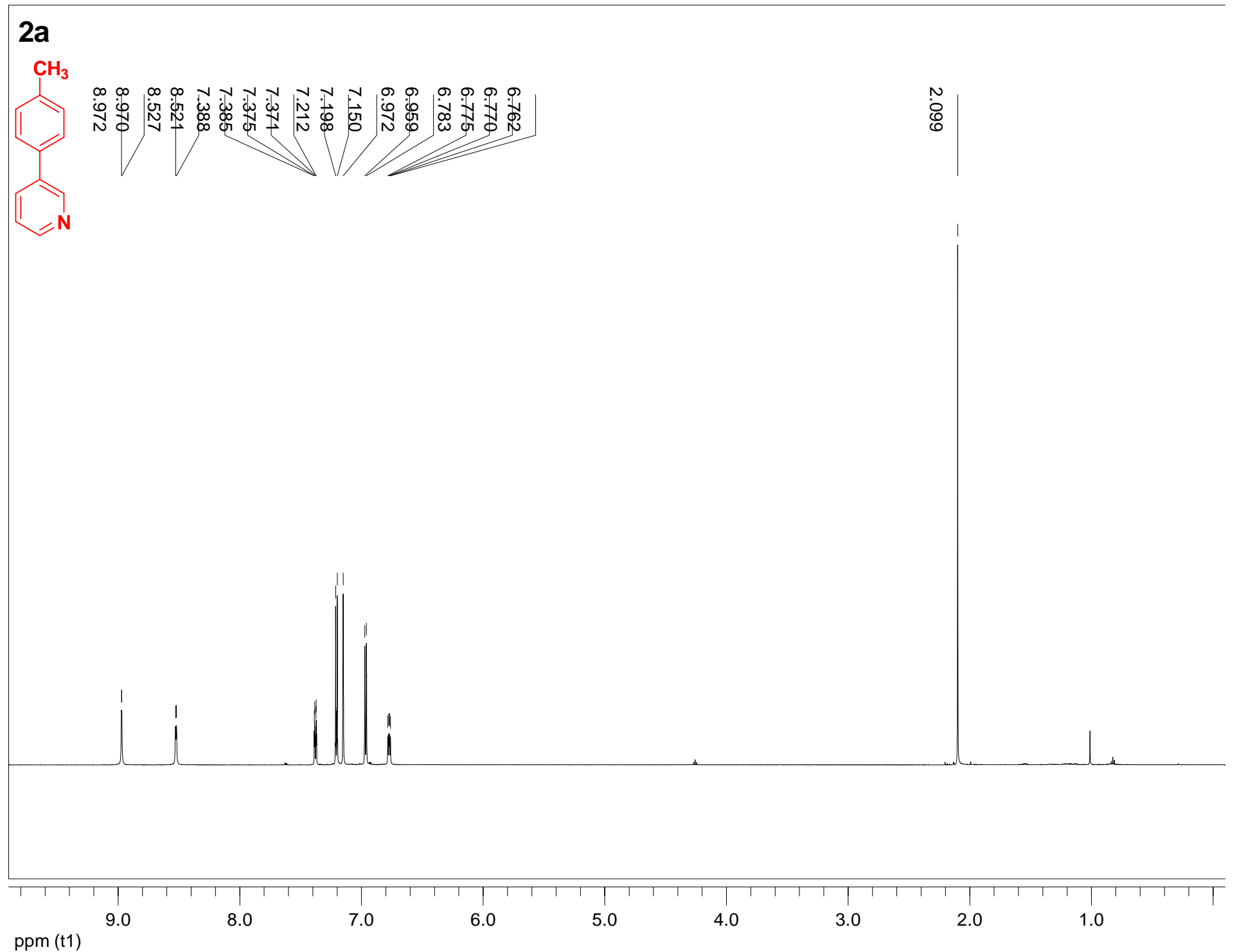


$2 a$

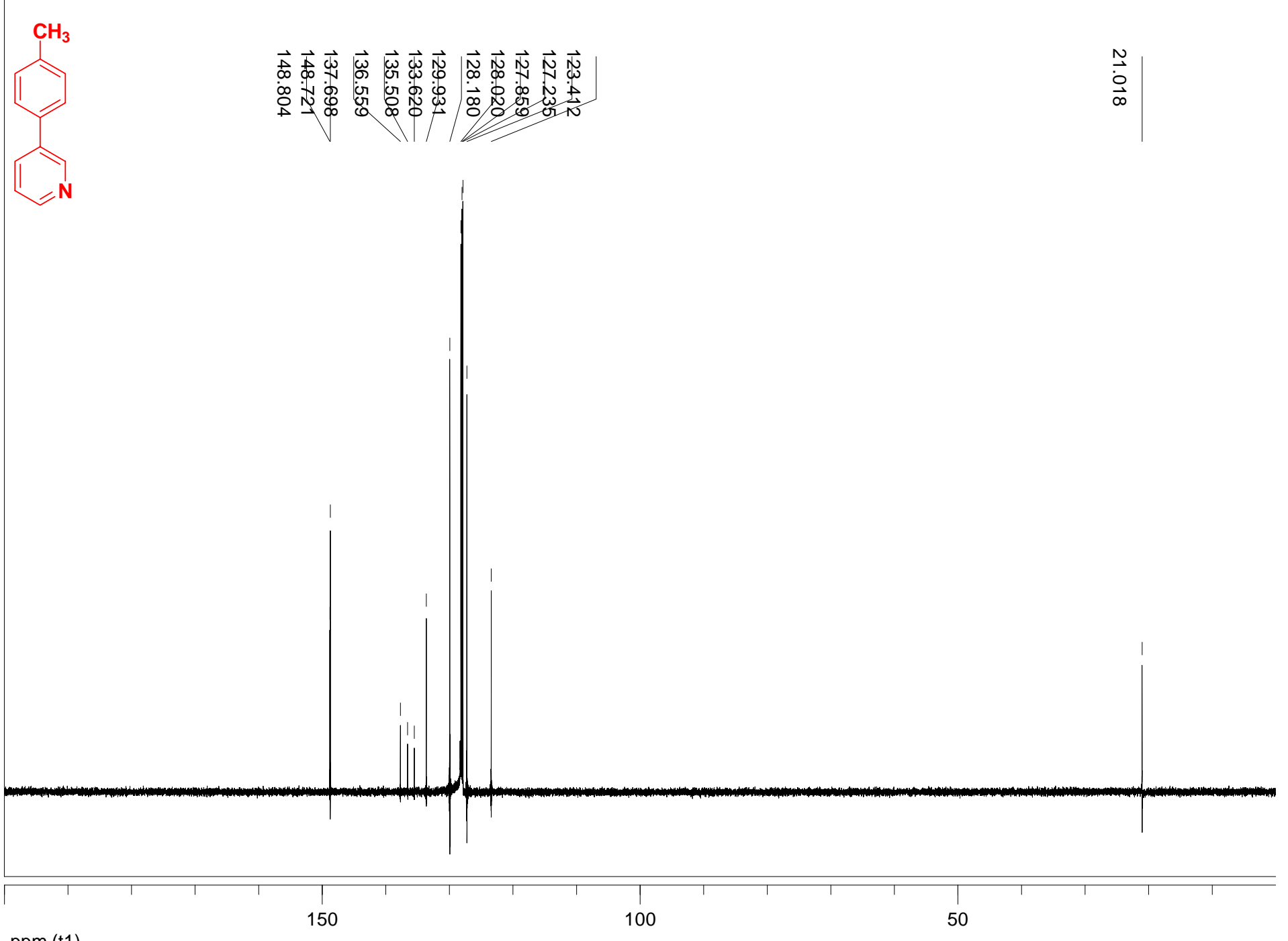

ppm (t1) 
2b

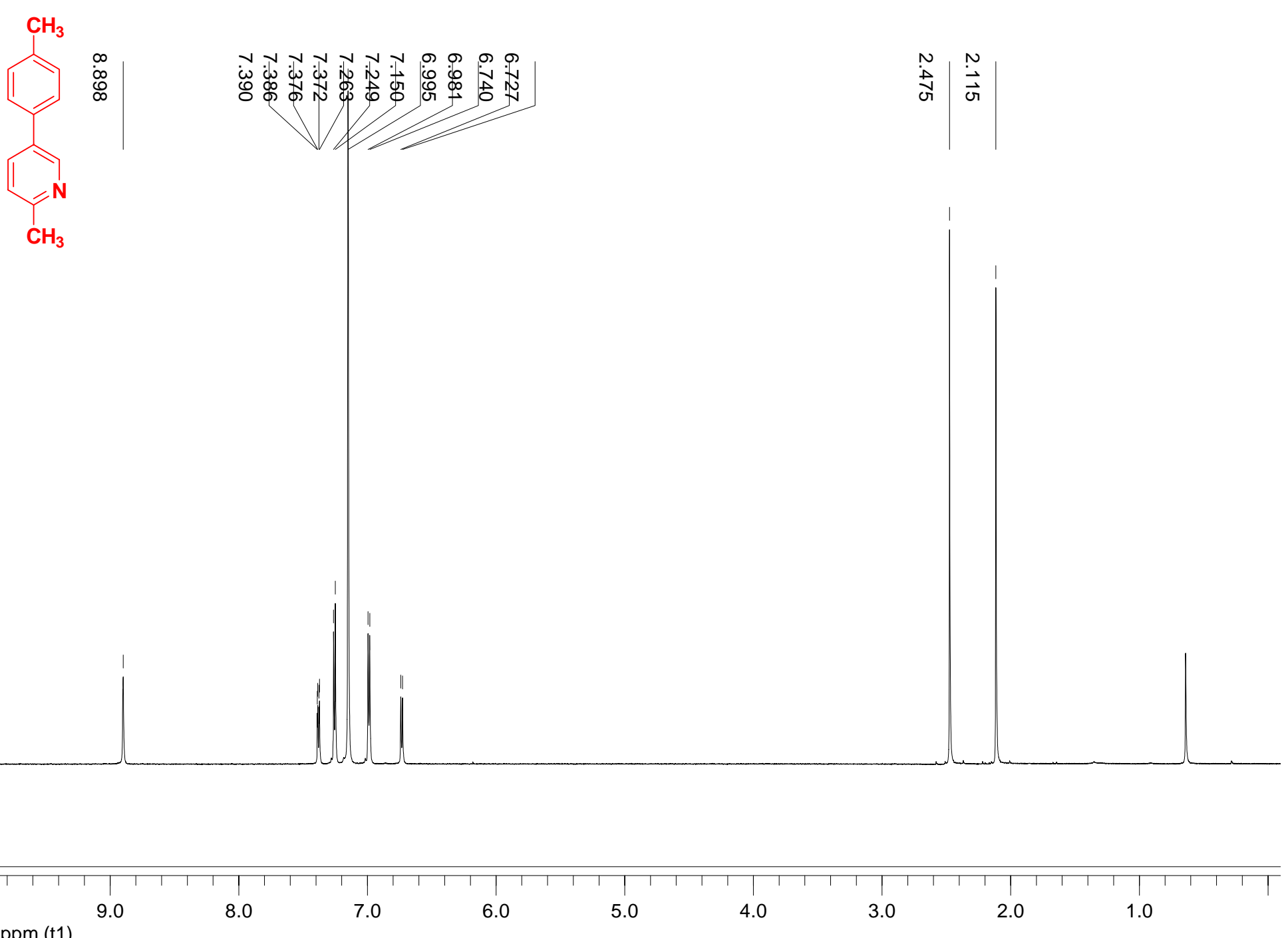




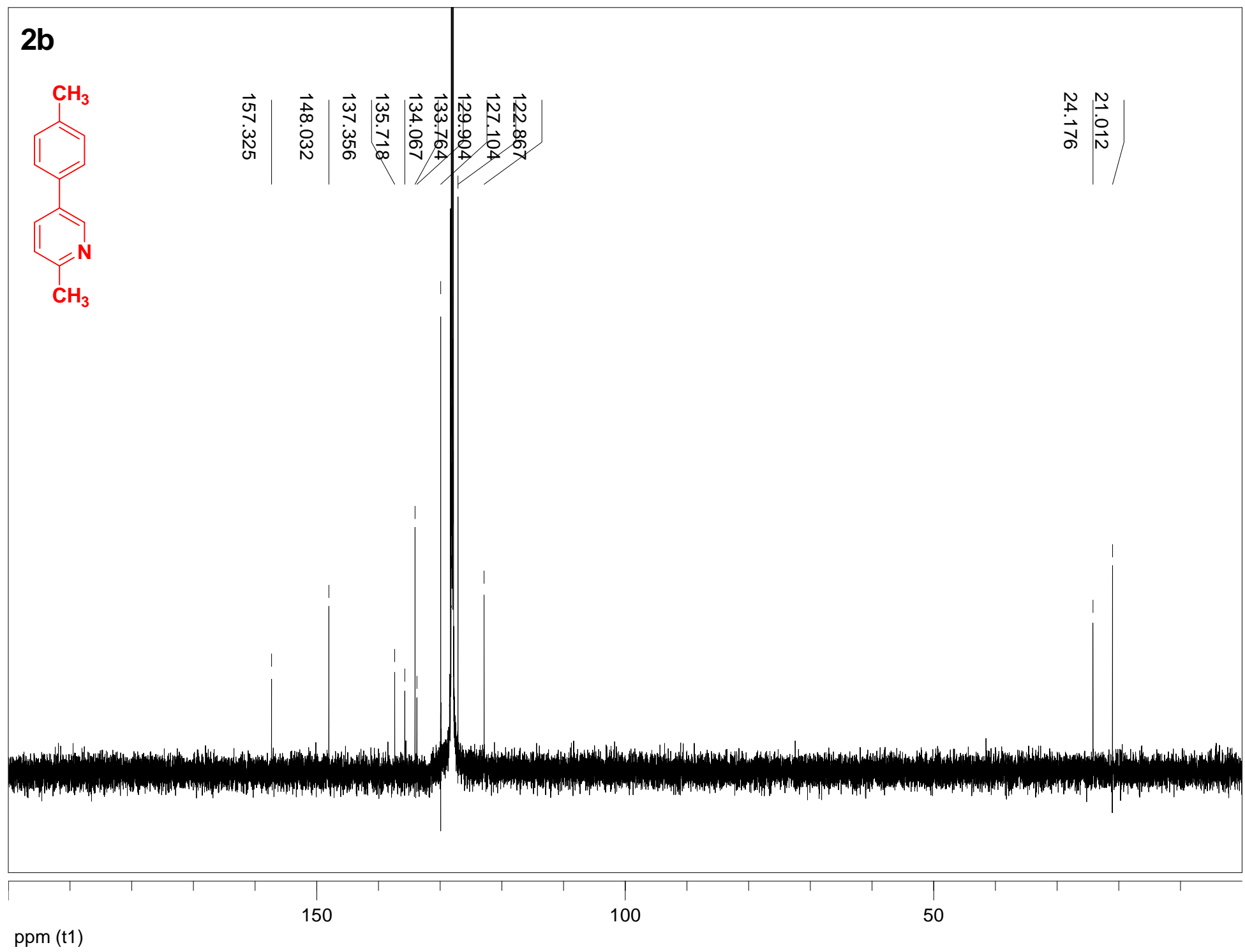


2c

$\mathrm{H}_{3} \mathrm{C}$

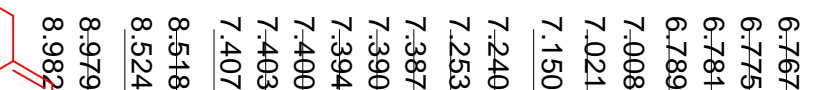

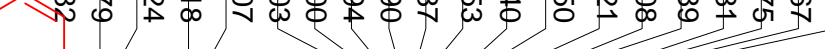

$i_{N=N}$

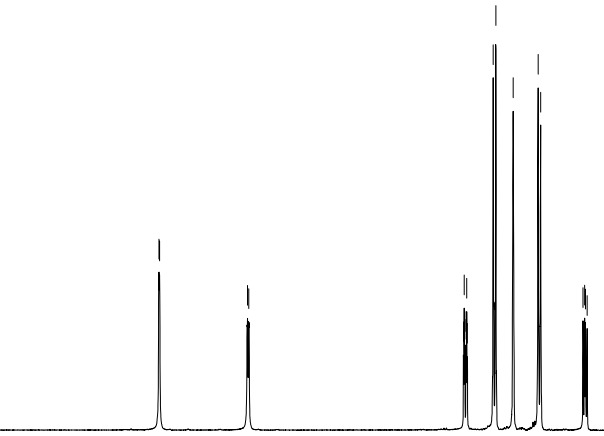

ppm (t1)

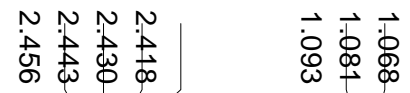

V 


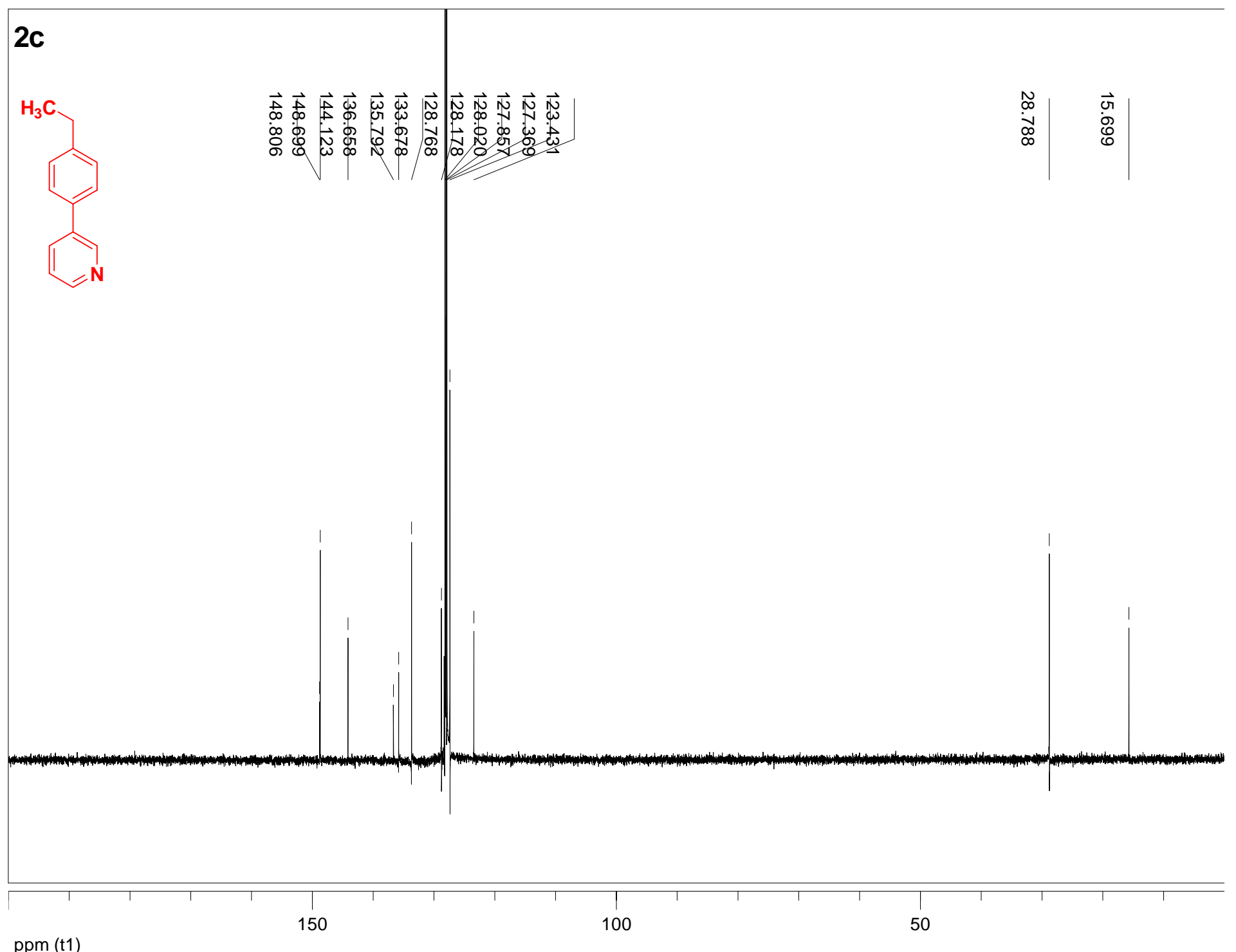


2d

$\mathrm{H}_{3} \mathrm{C}$

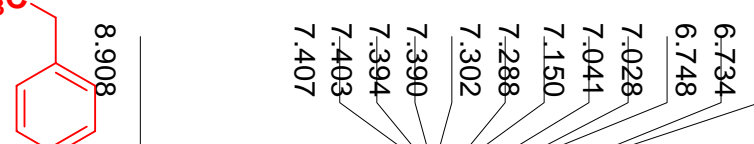

$\mathrm{CH}_{3}^{\mathrm{N}}$$$
\text { - }
$$
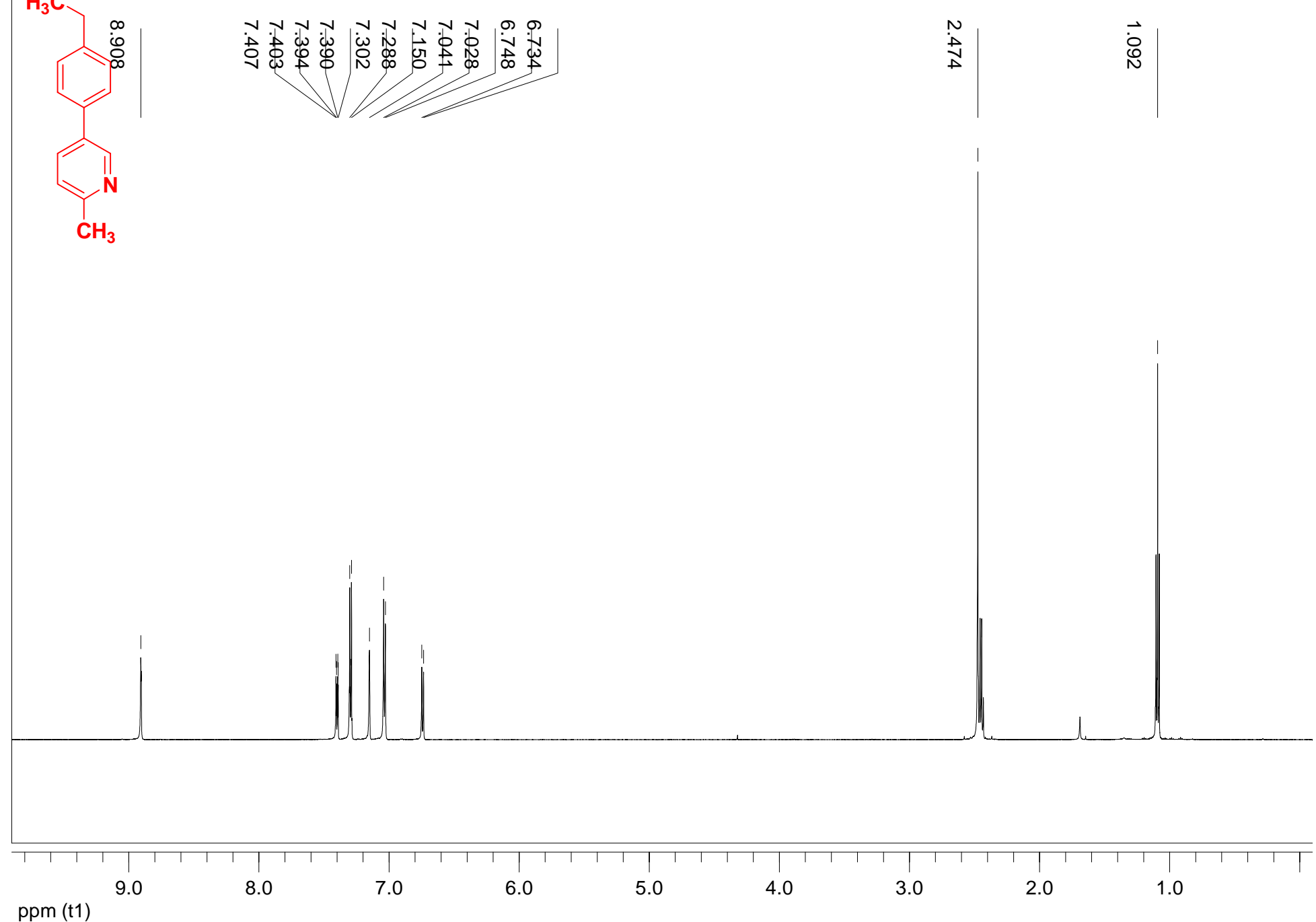


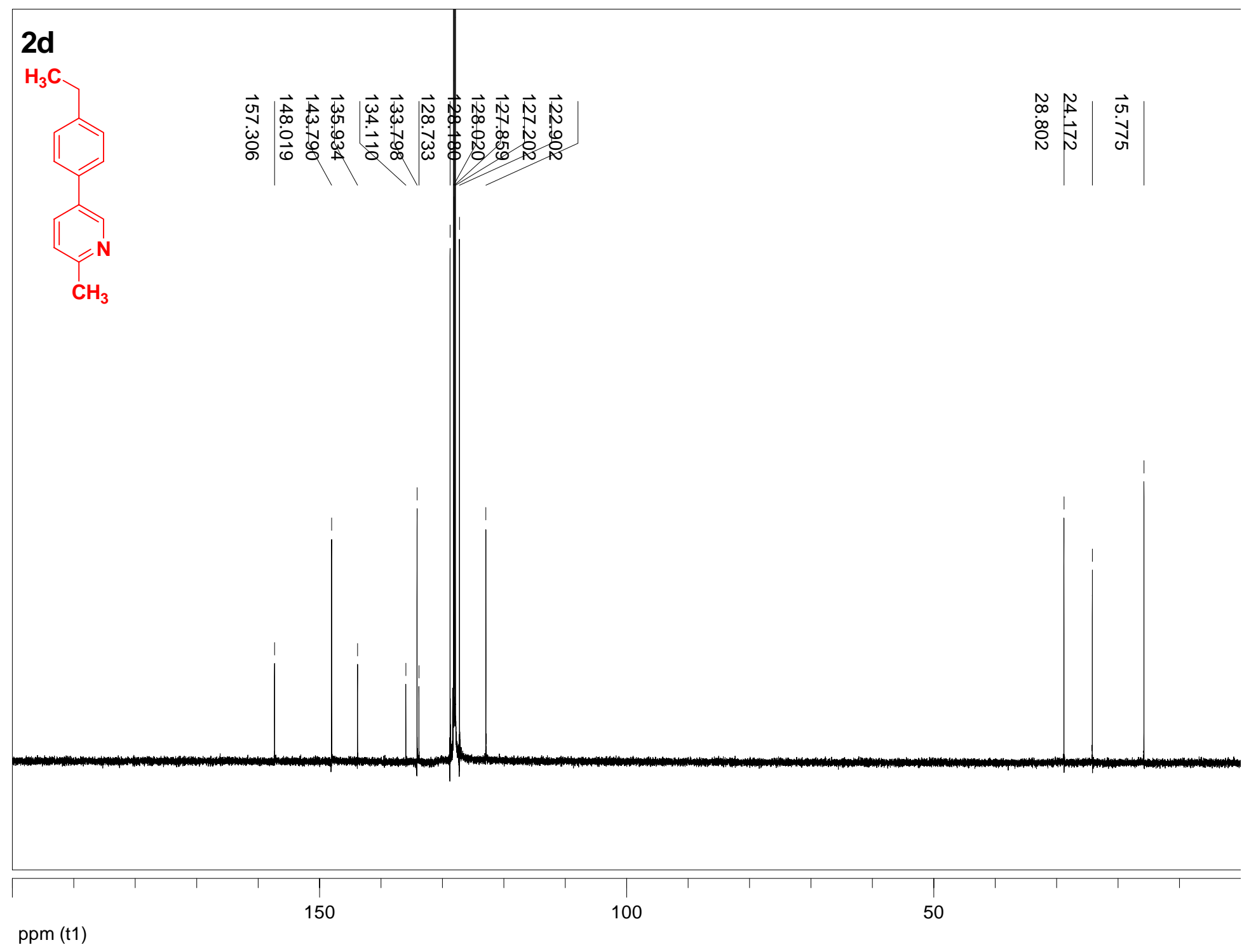


$2 \mathrm{e}$

$\mathrm{CH}_{3}$

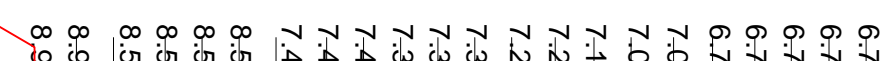

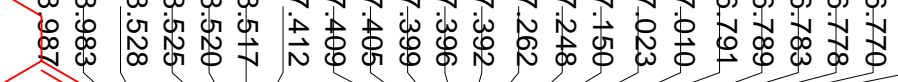
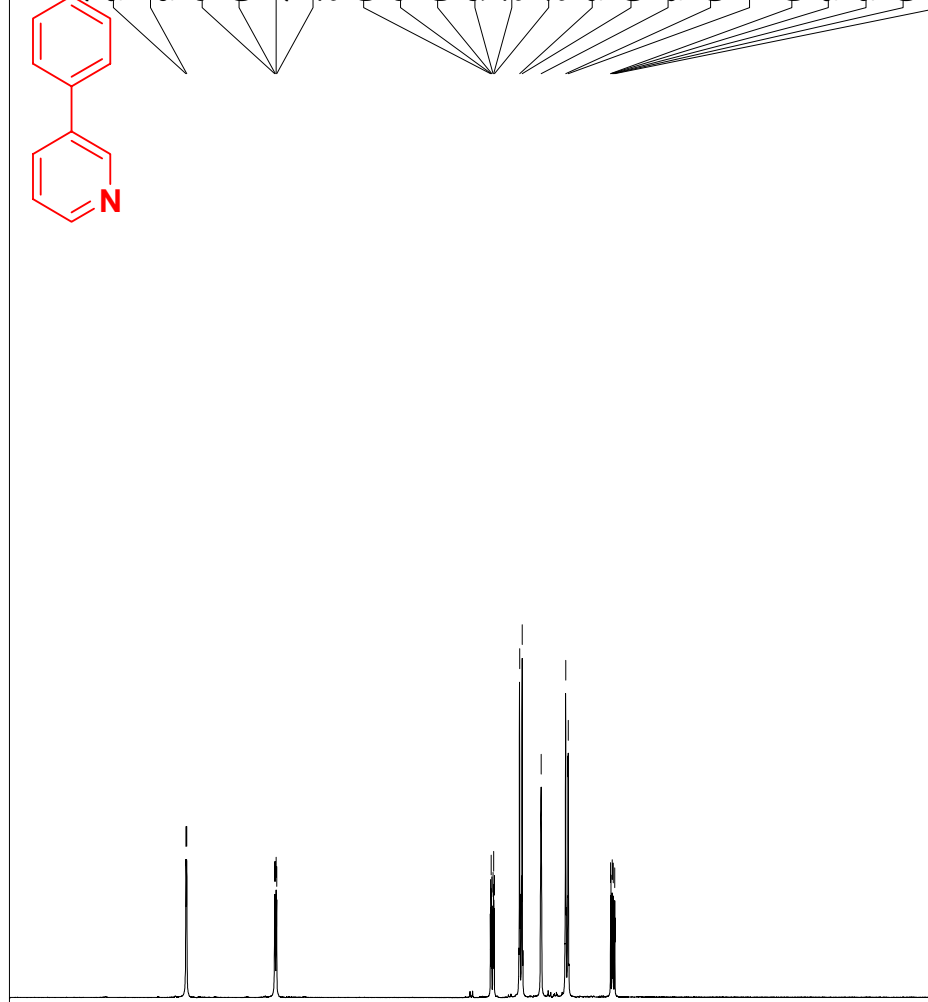

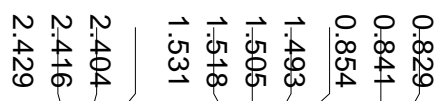
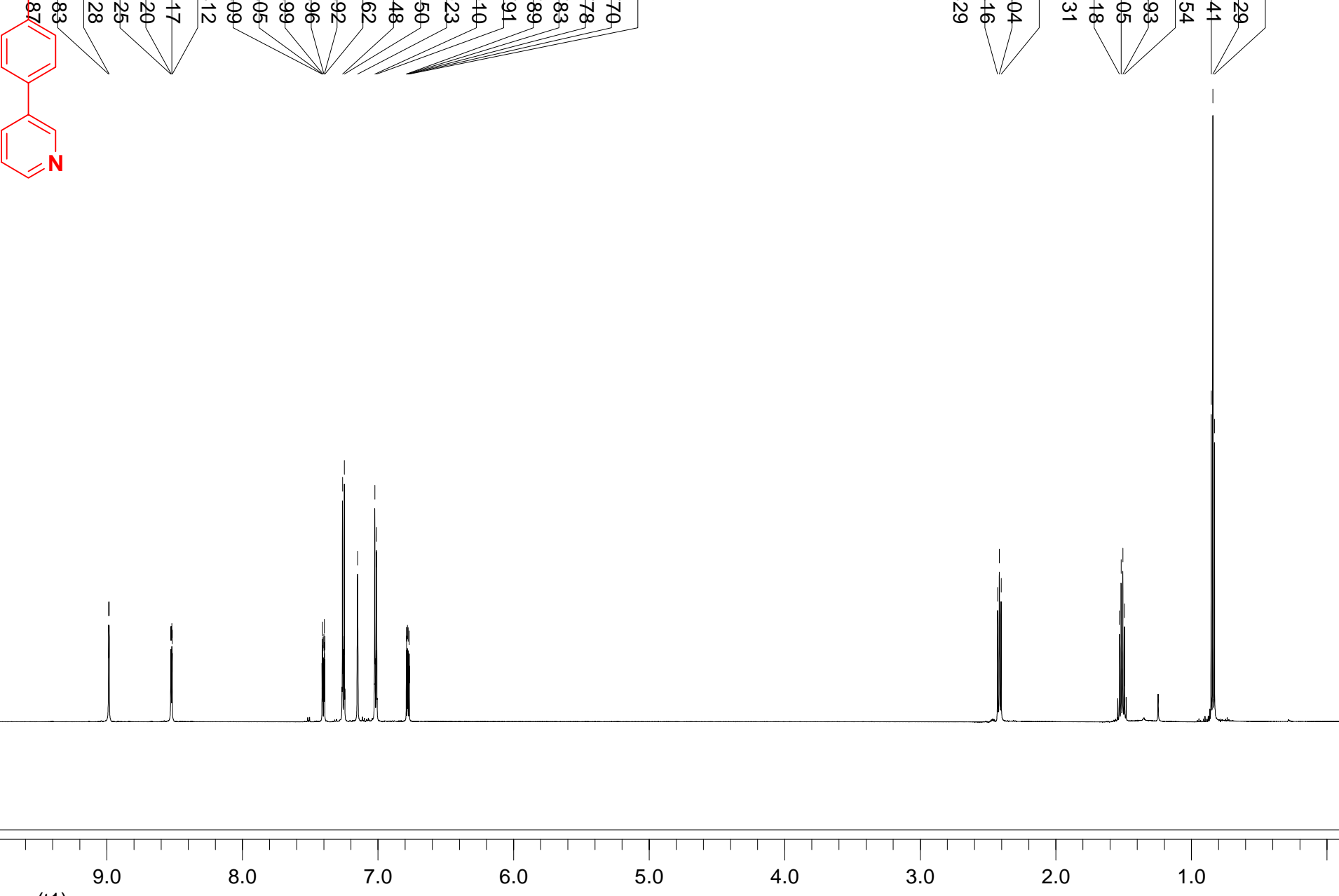

ppm (t1) 


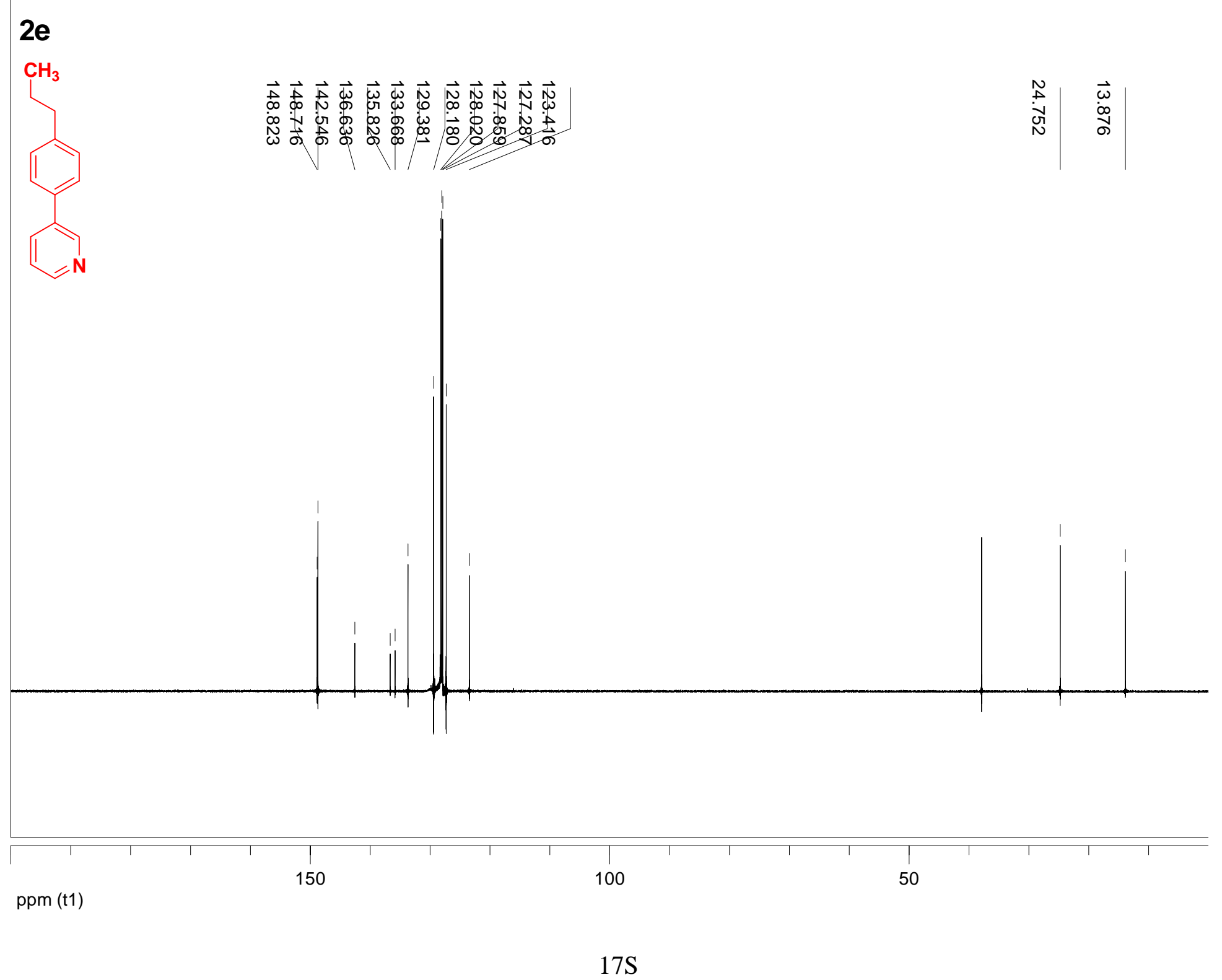




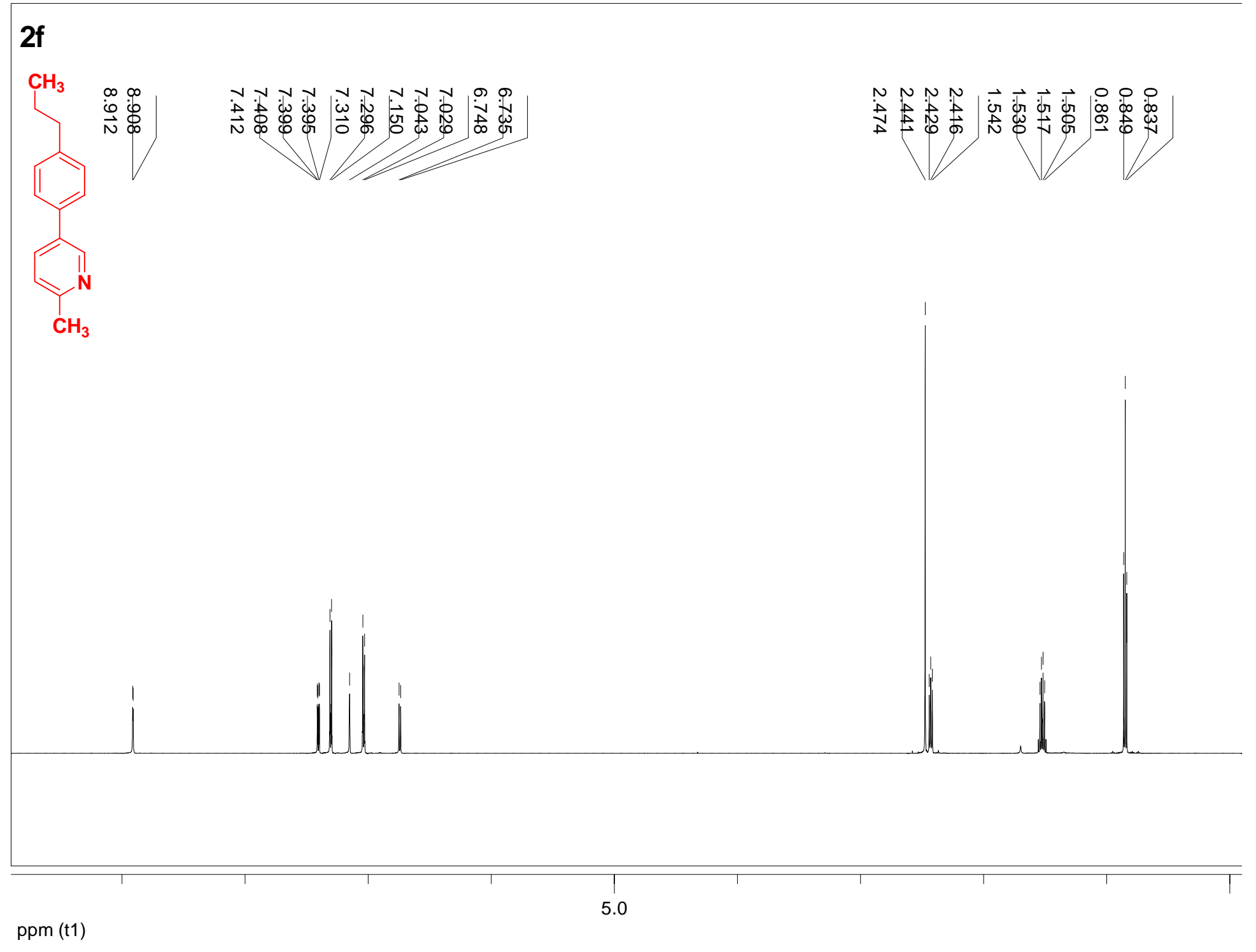




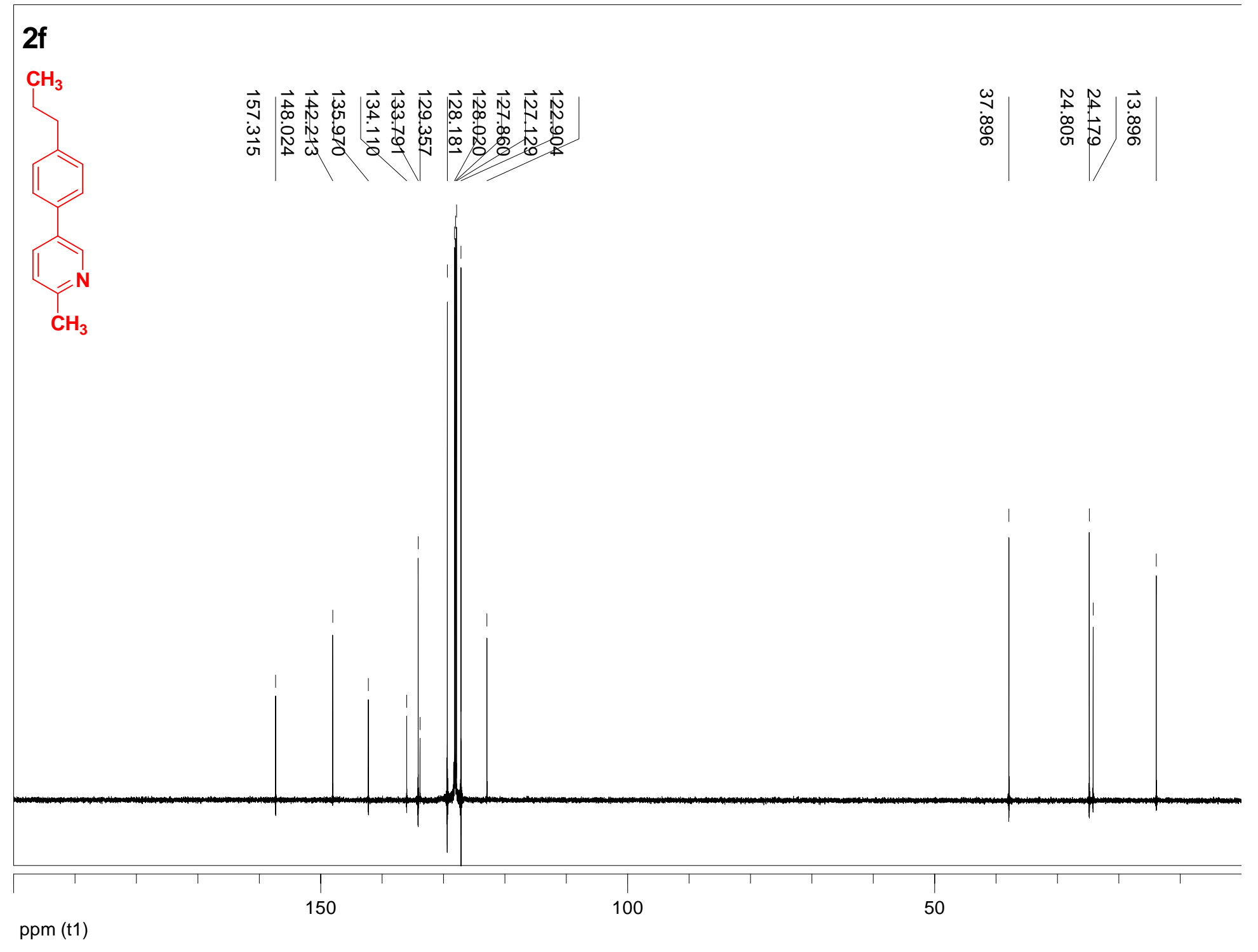




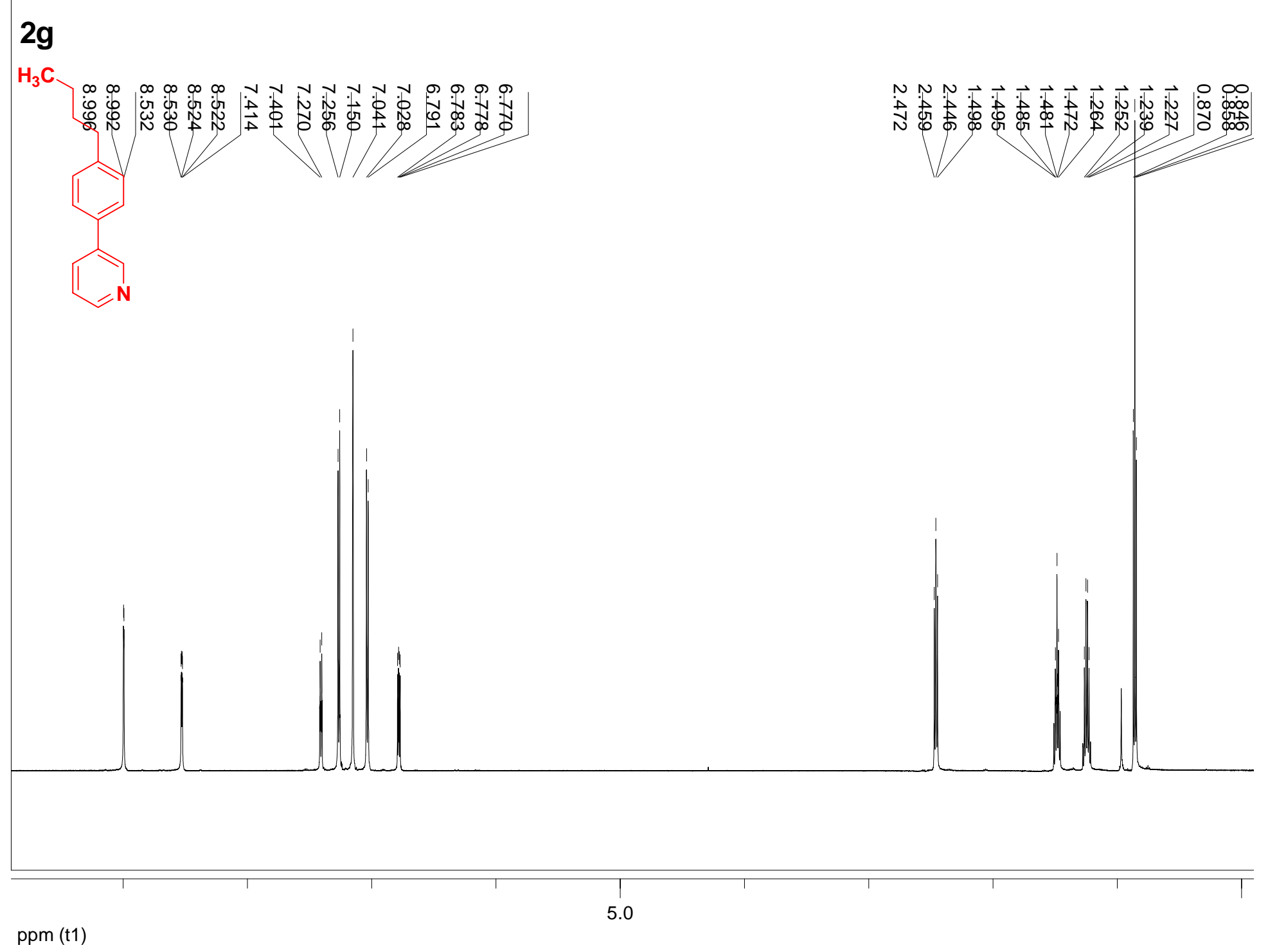




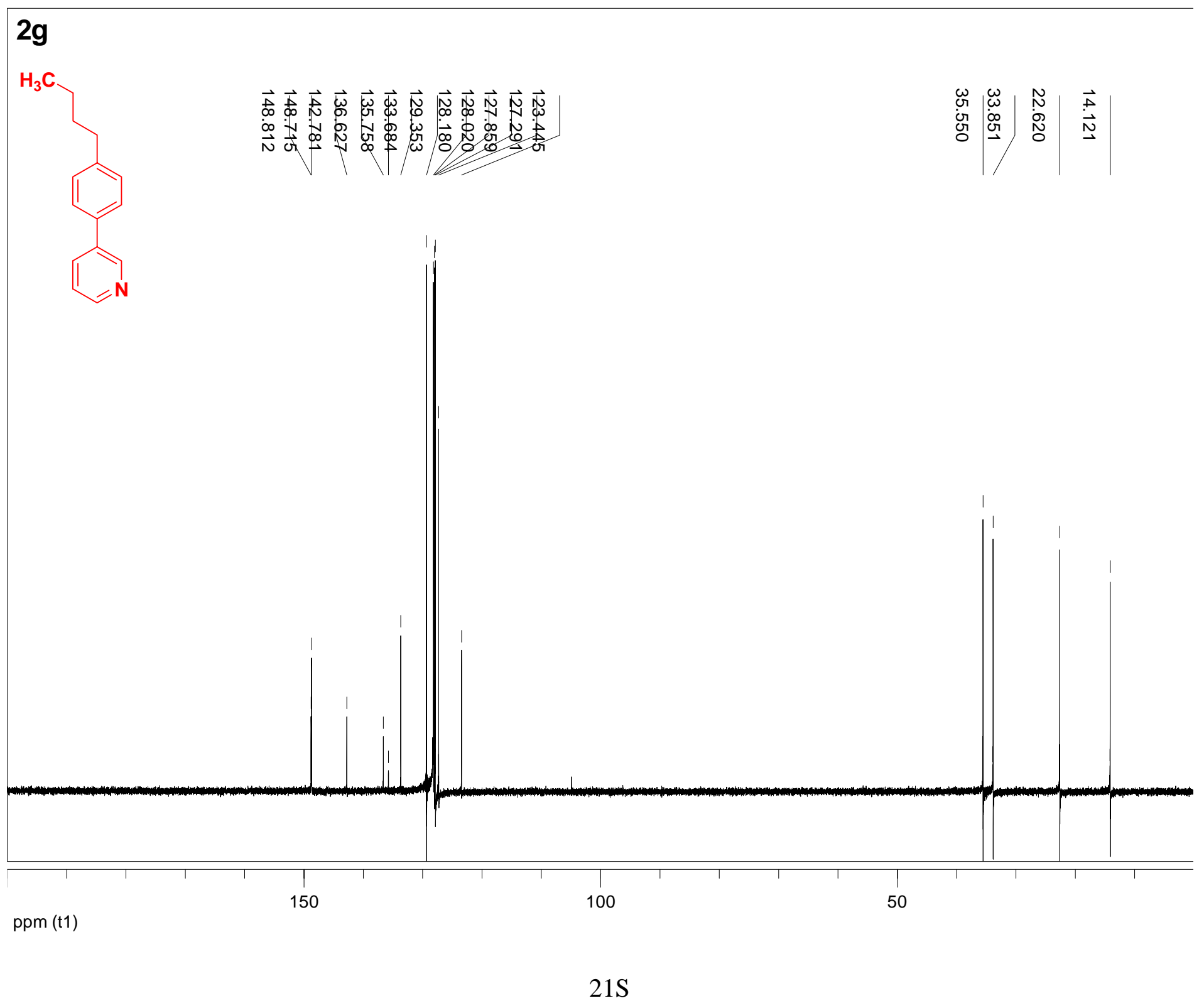


3b

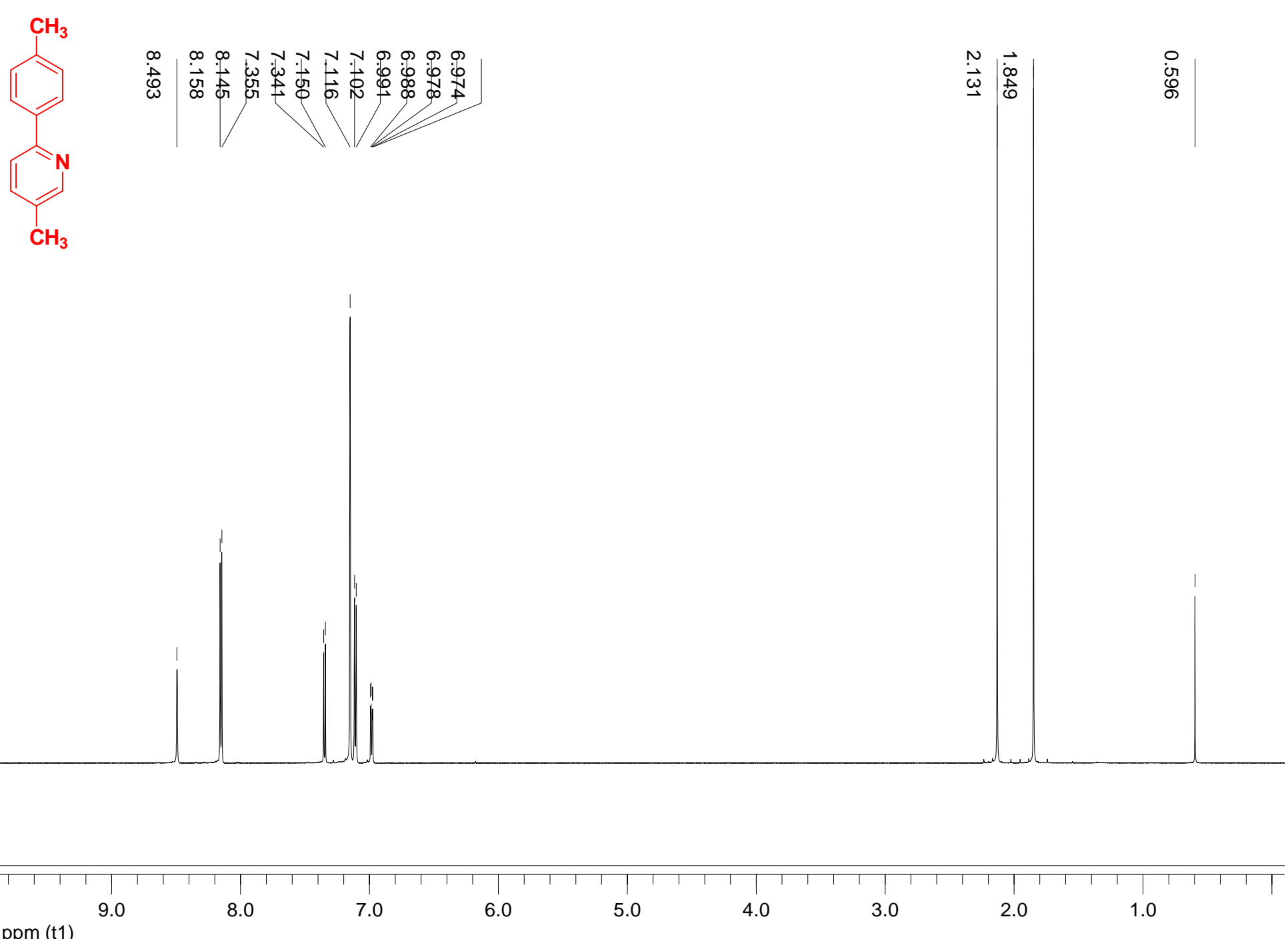

ppm (t1) 


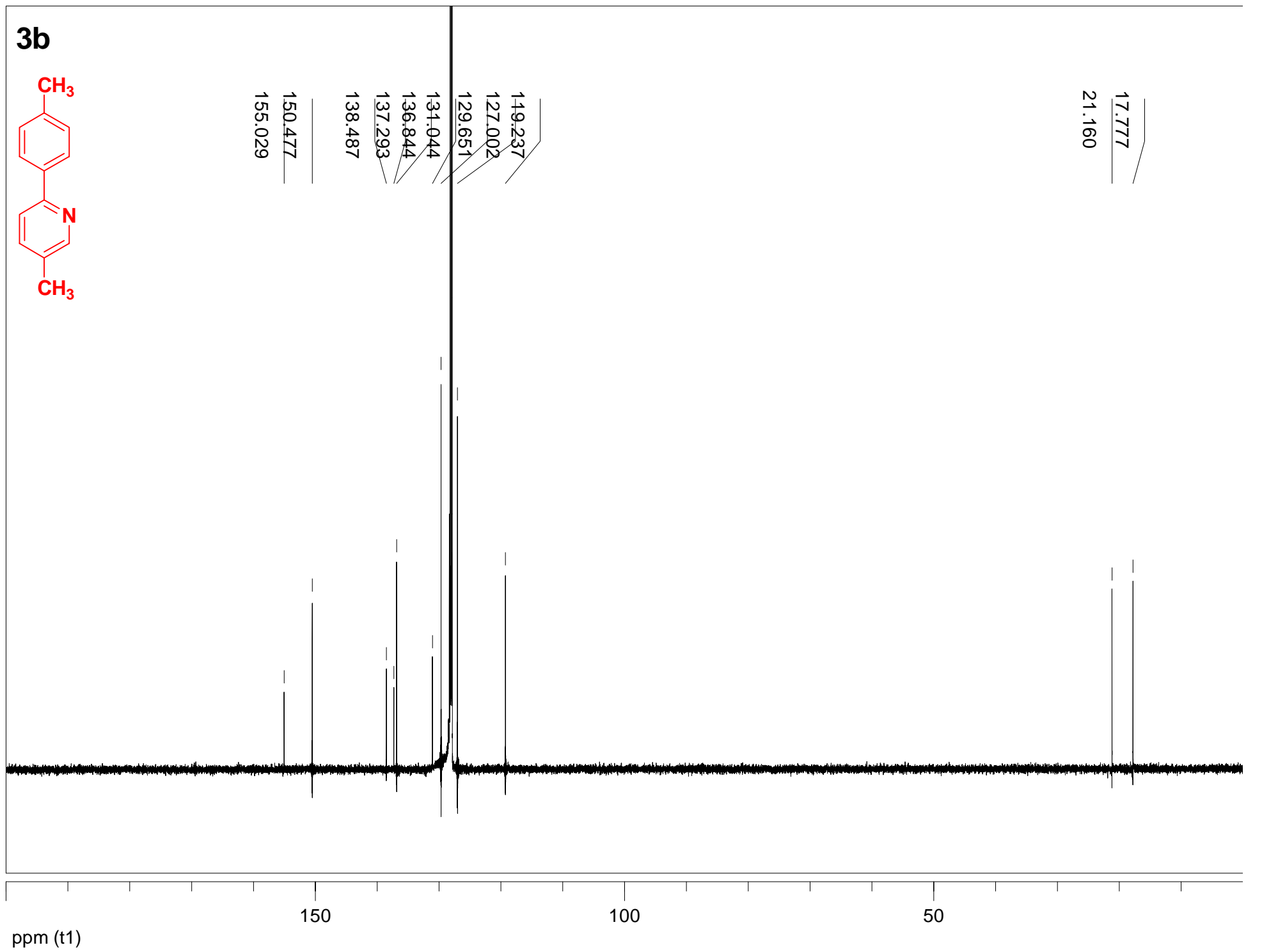




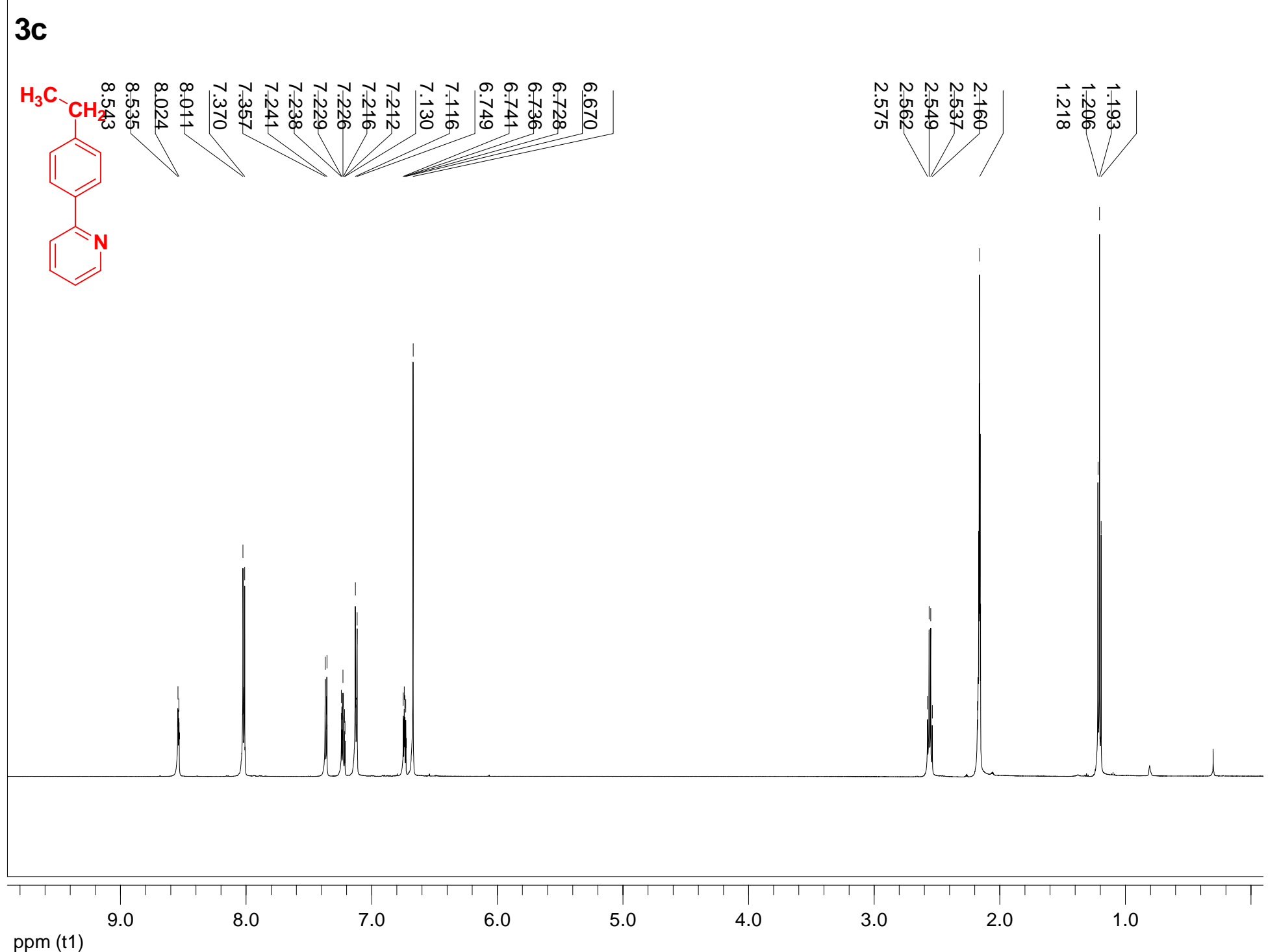




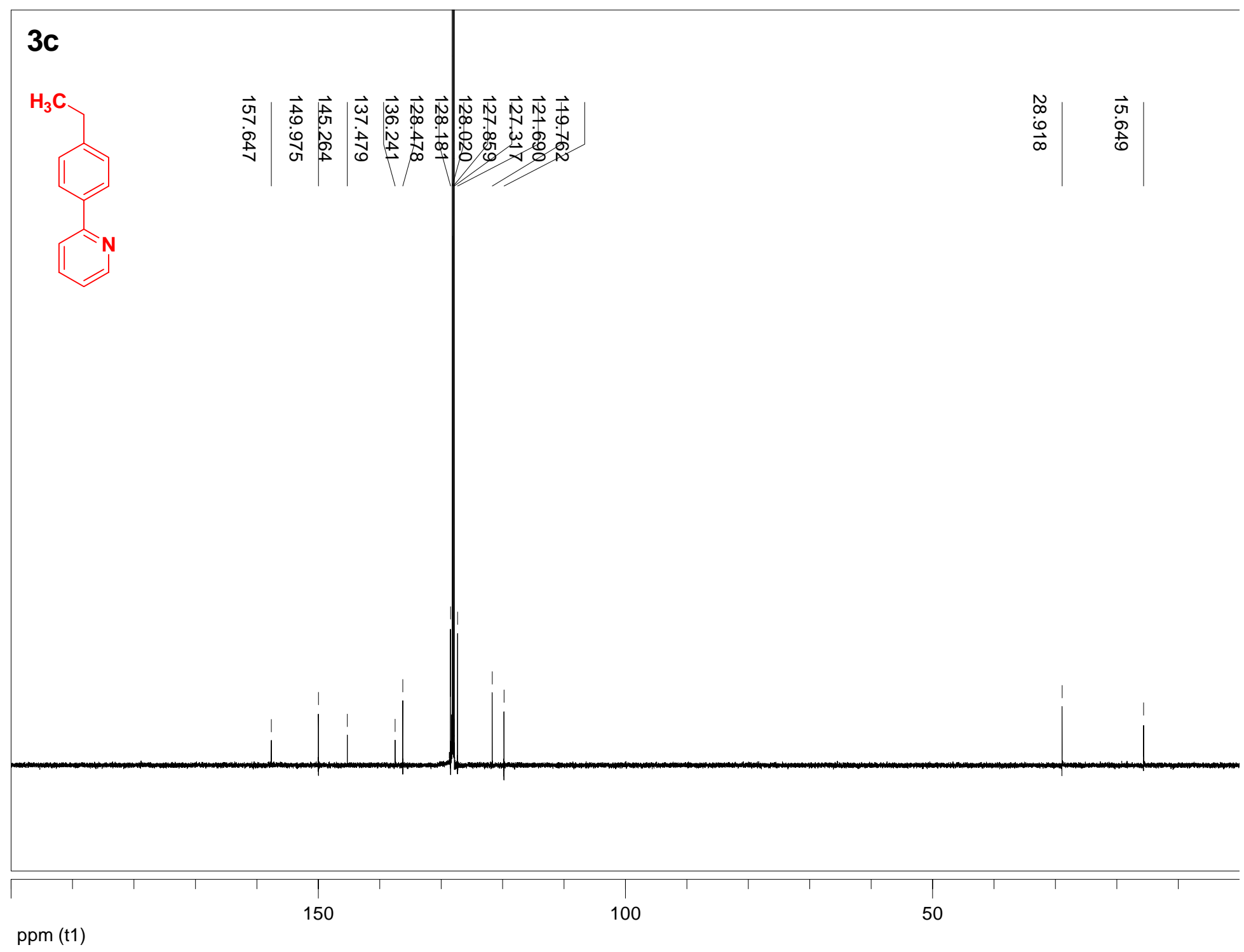




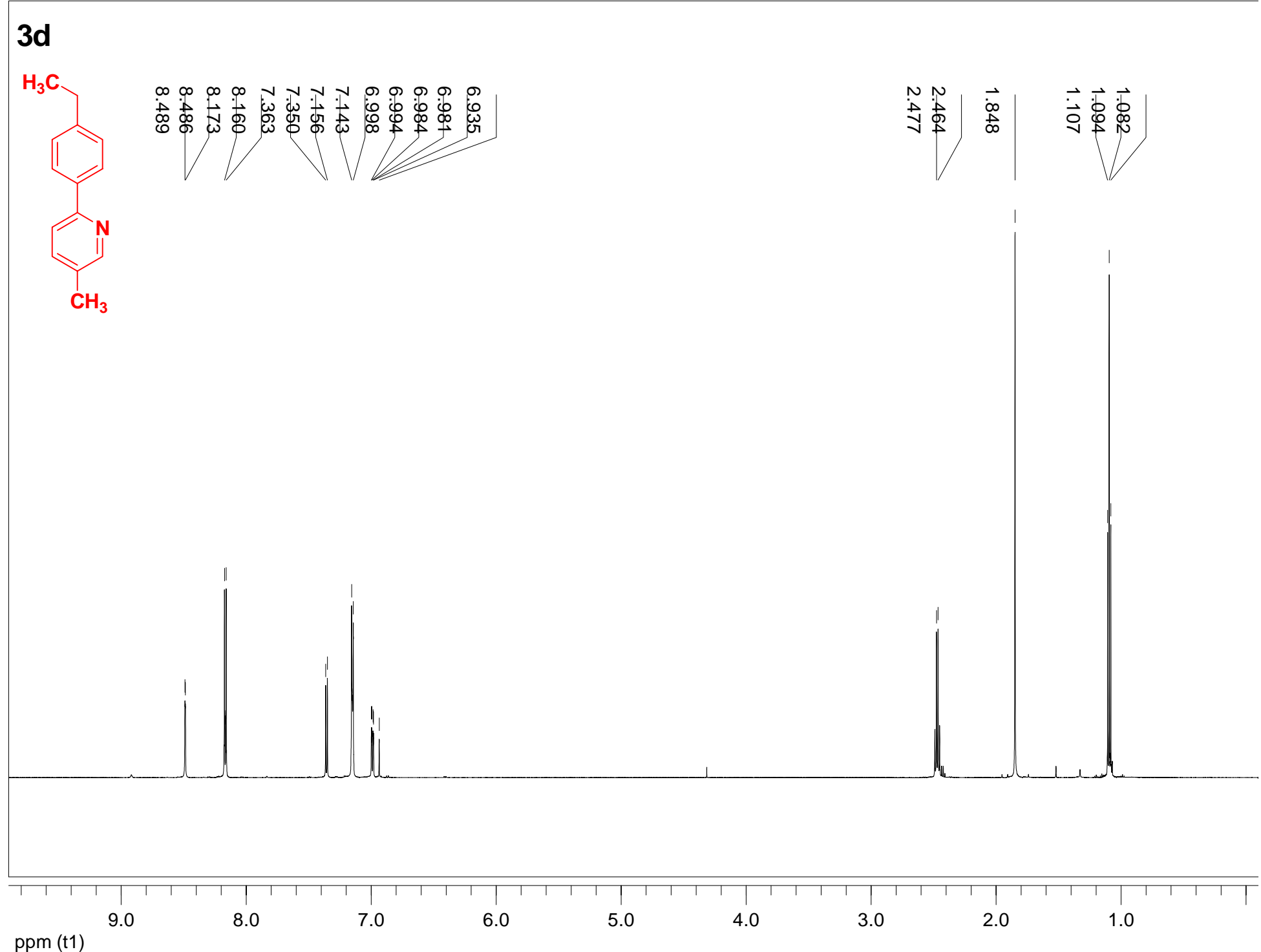




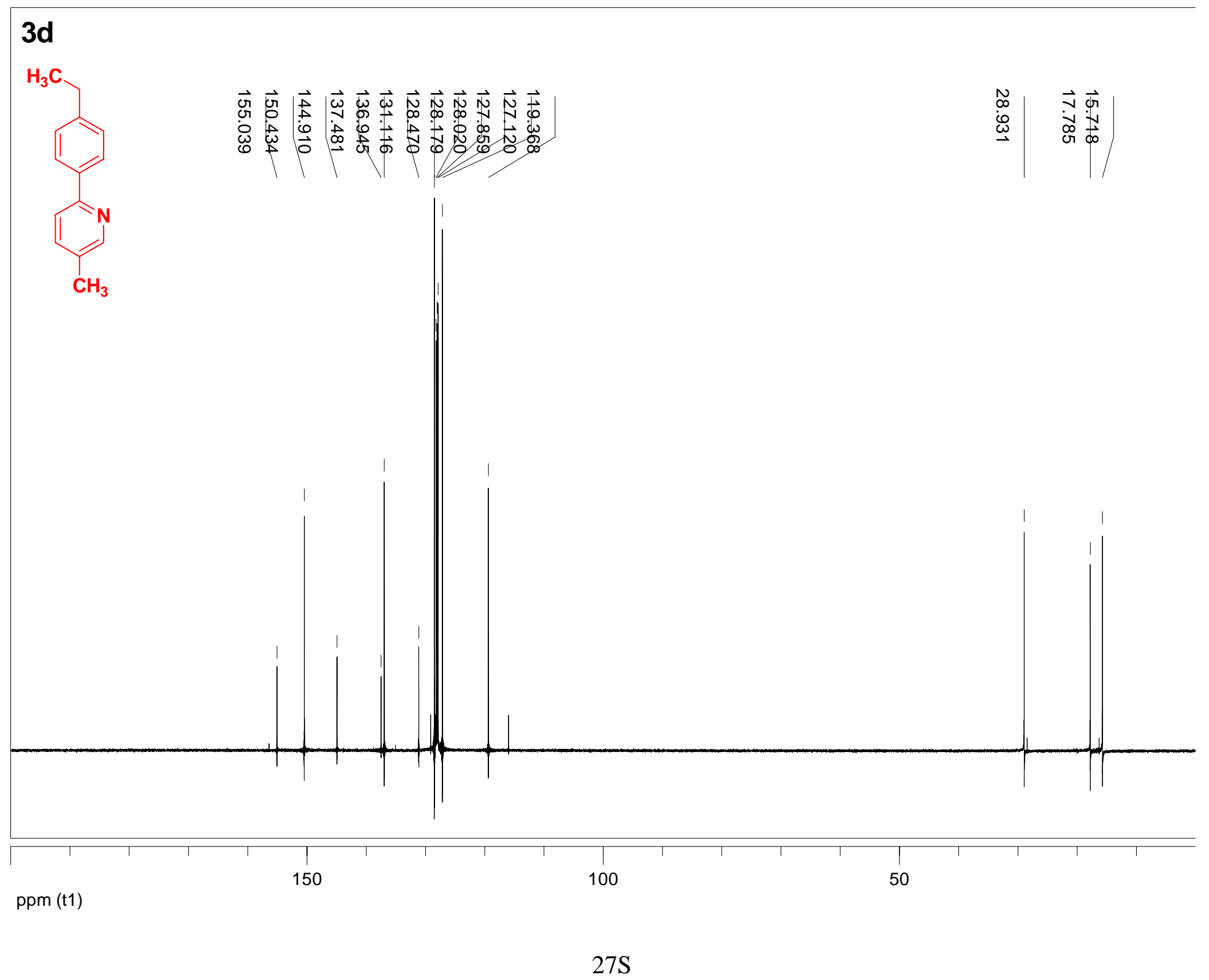




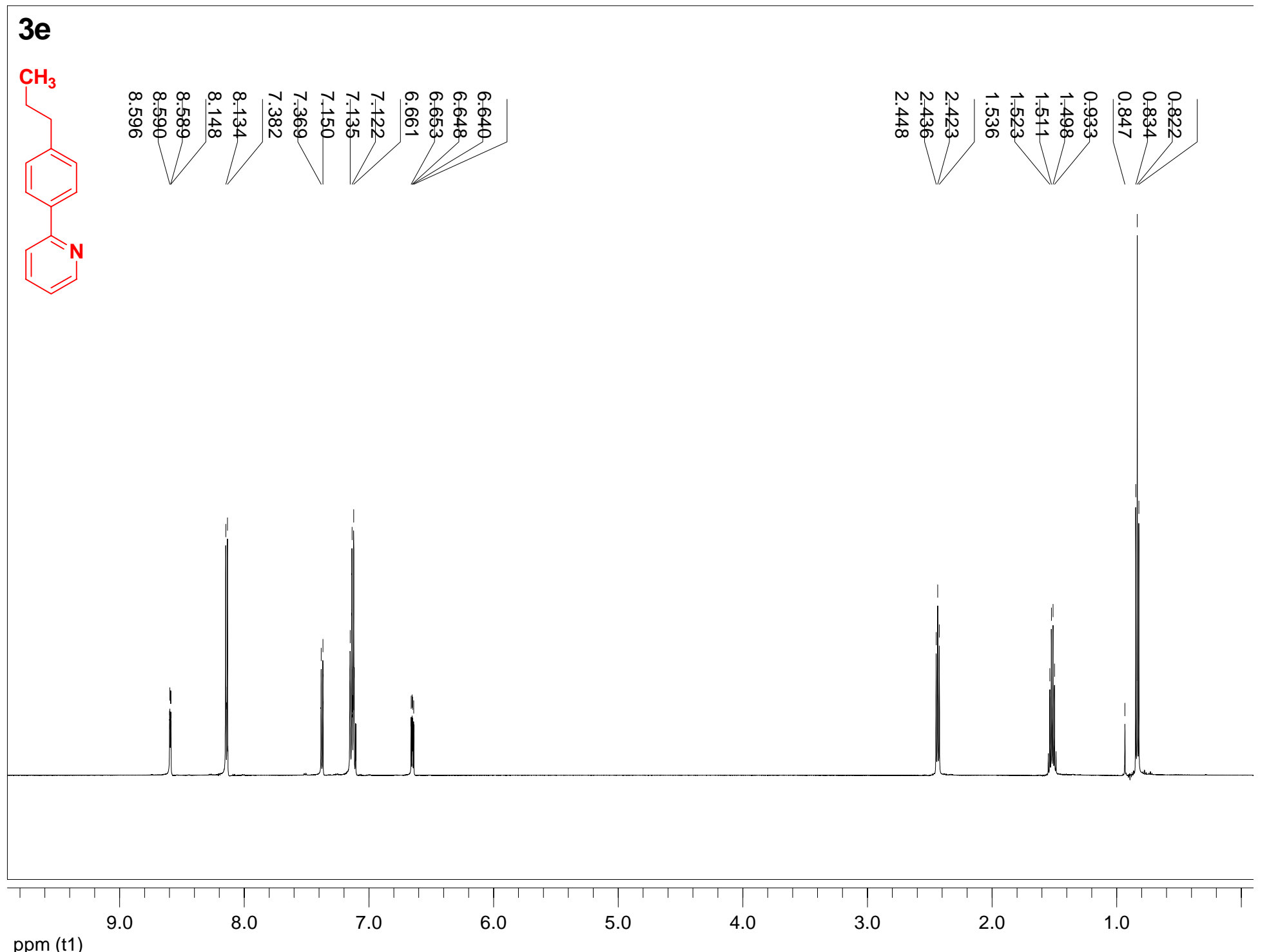




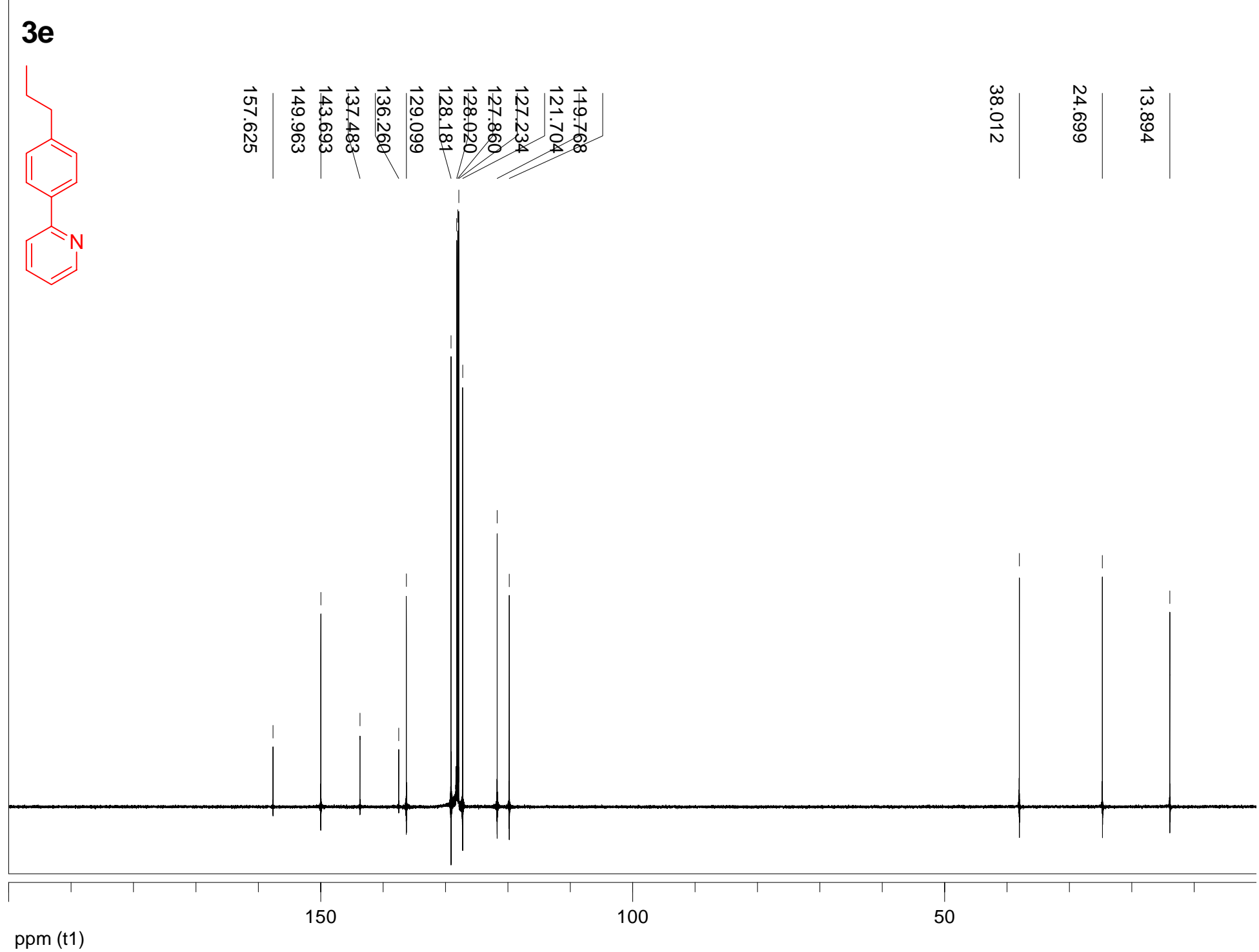




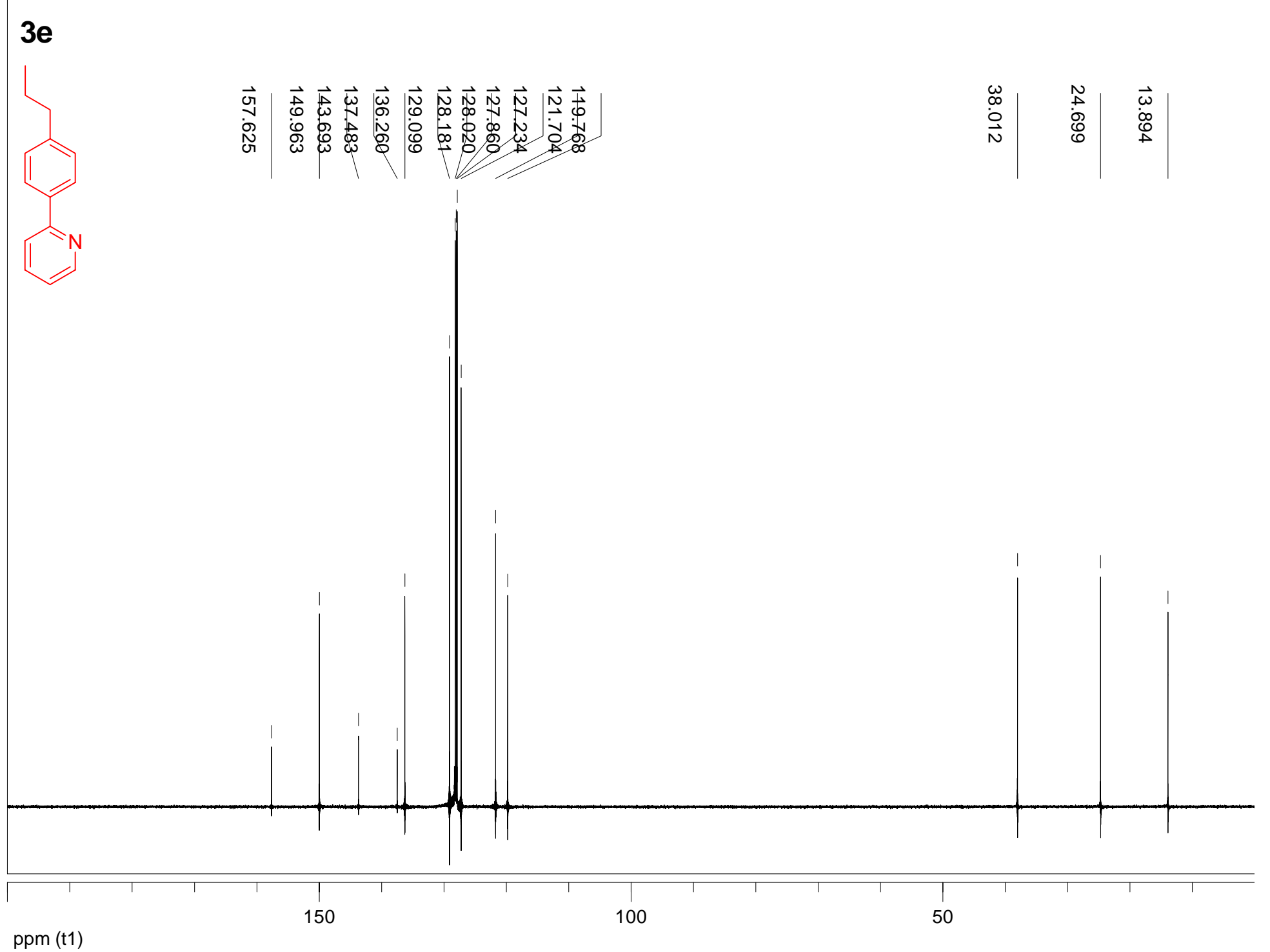




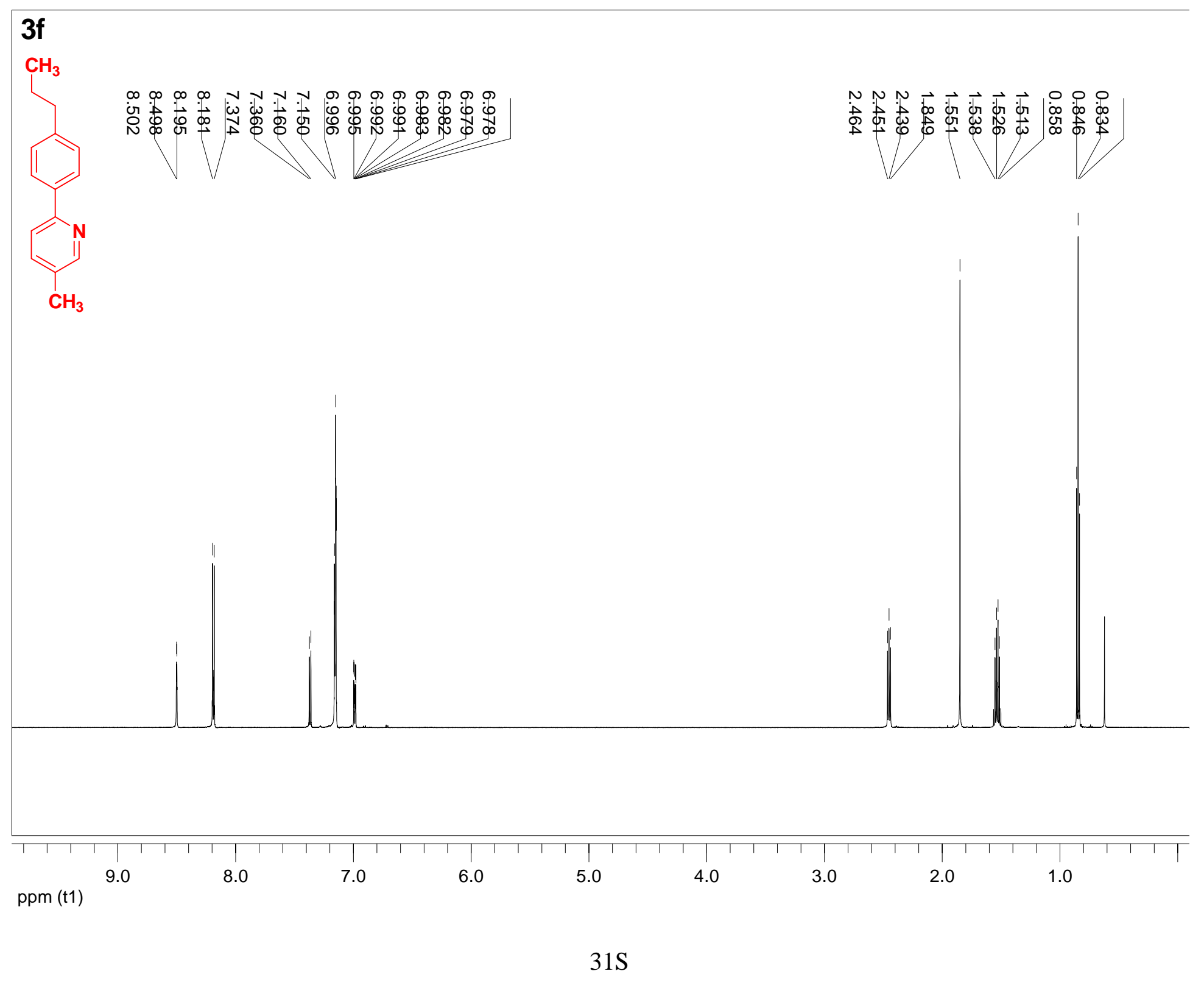




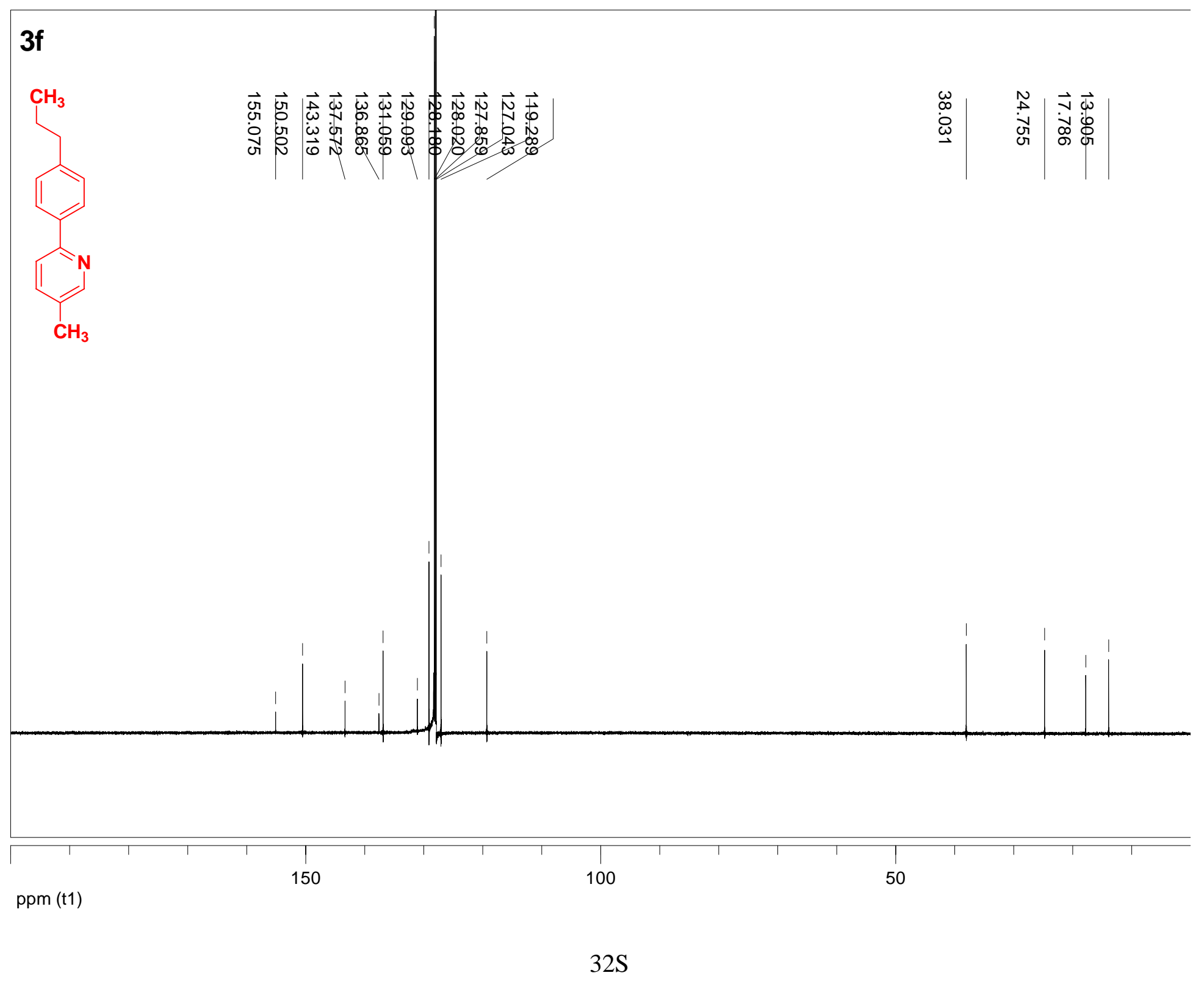




\section{$3 g$}

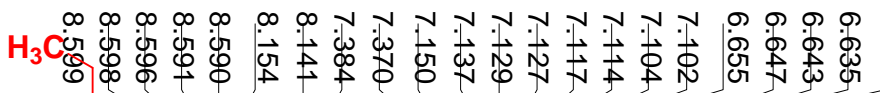

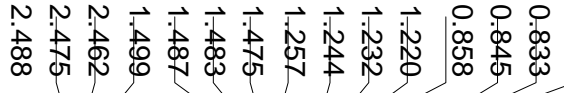

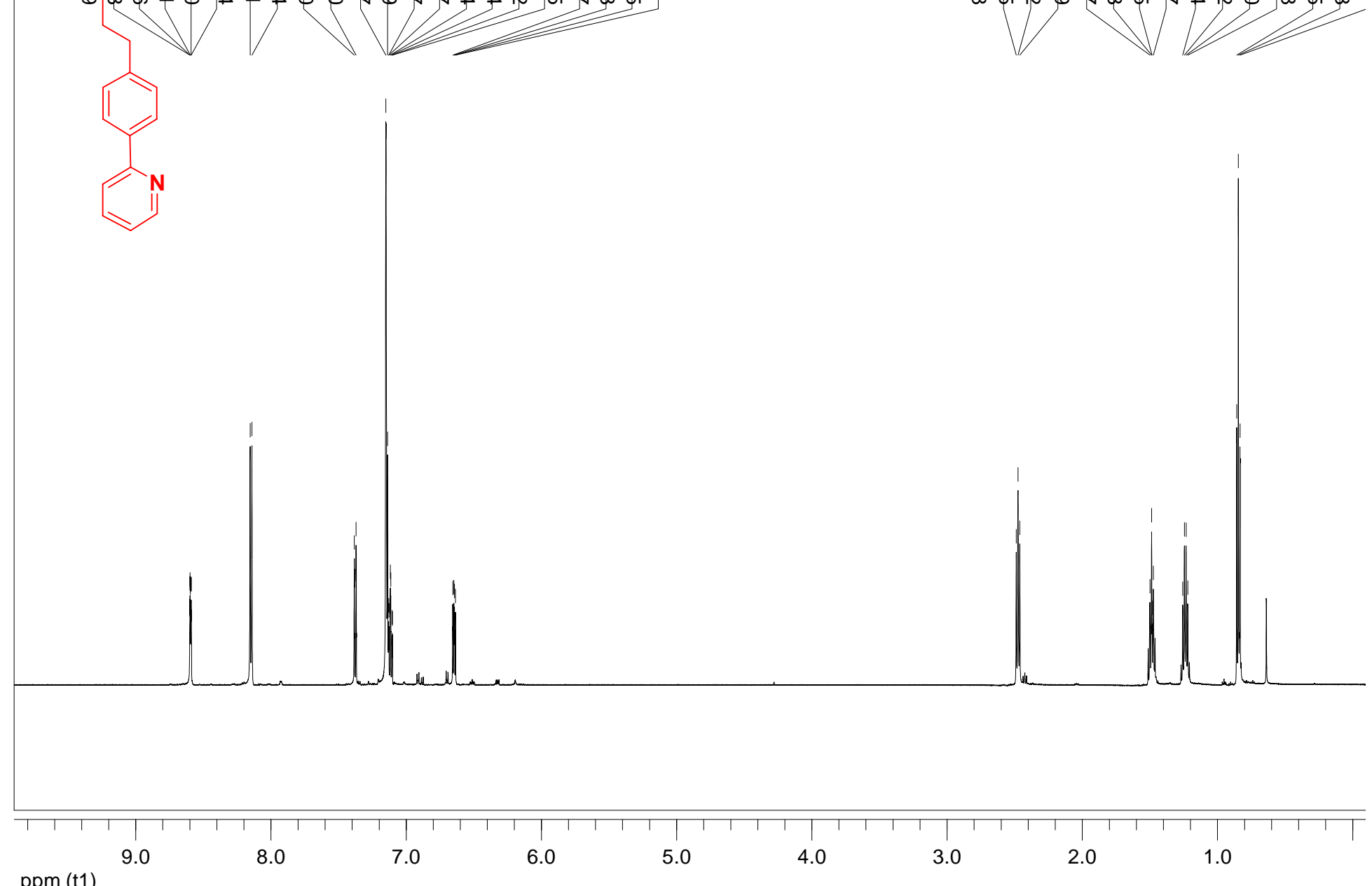




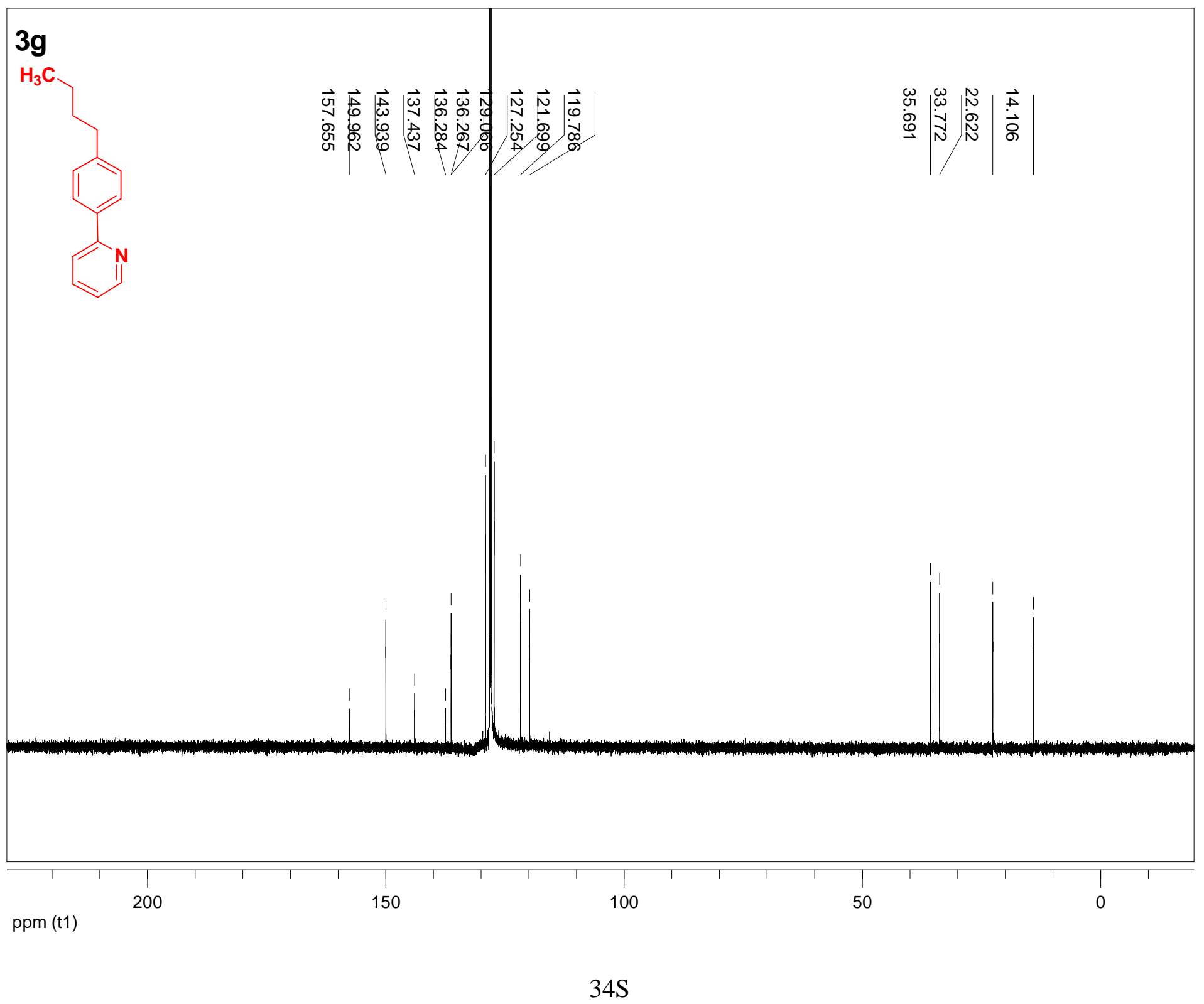




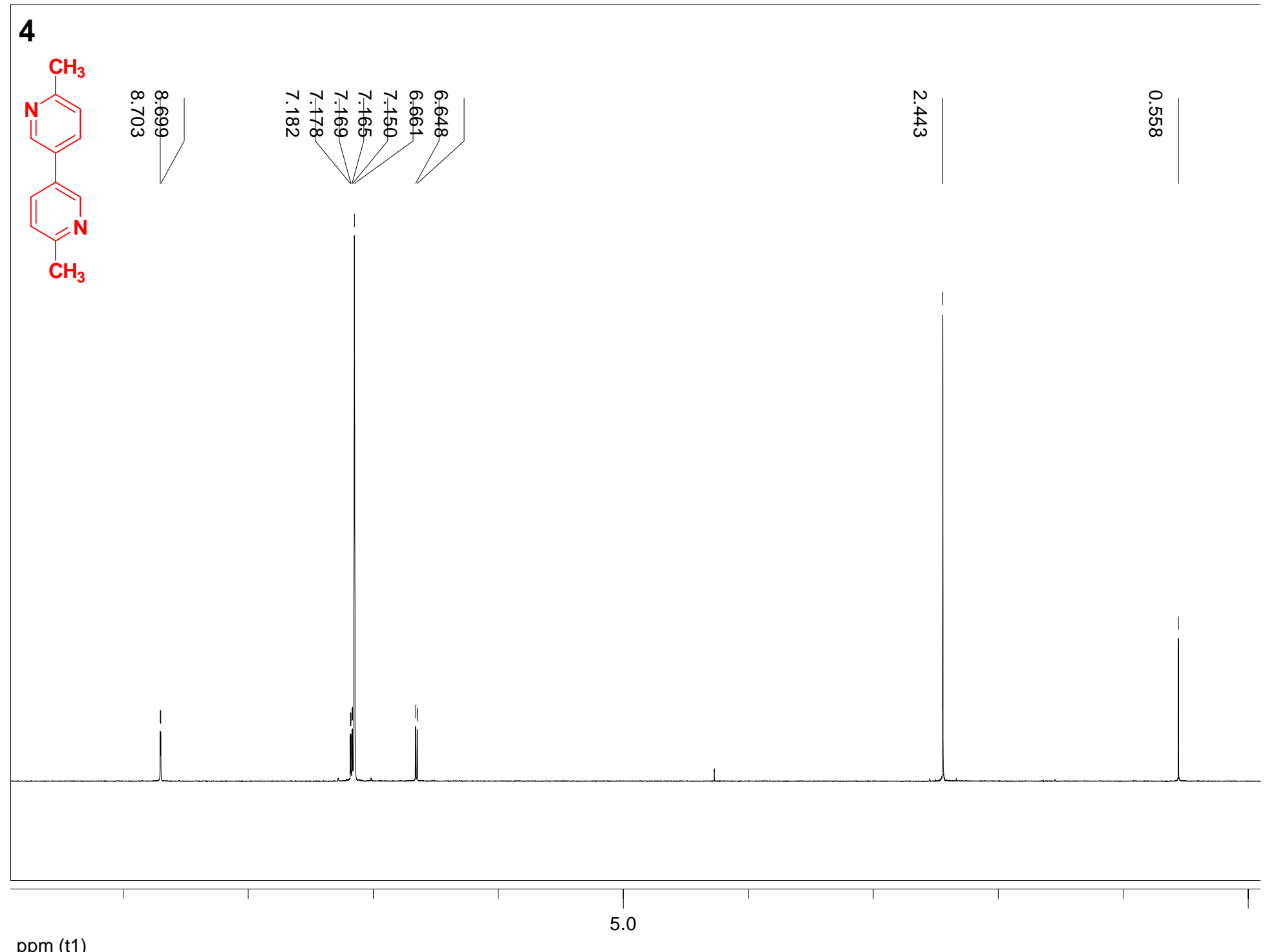




\section{4}

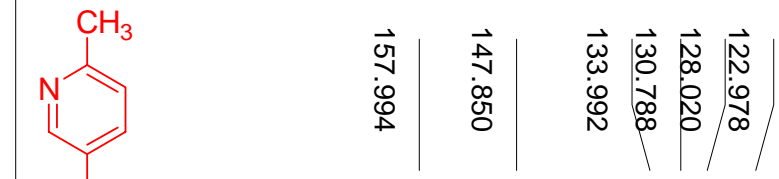

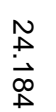

$\mathrm{CH}_{\mathrm{CH}_{3}}^{\mathrm{N}}$
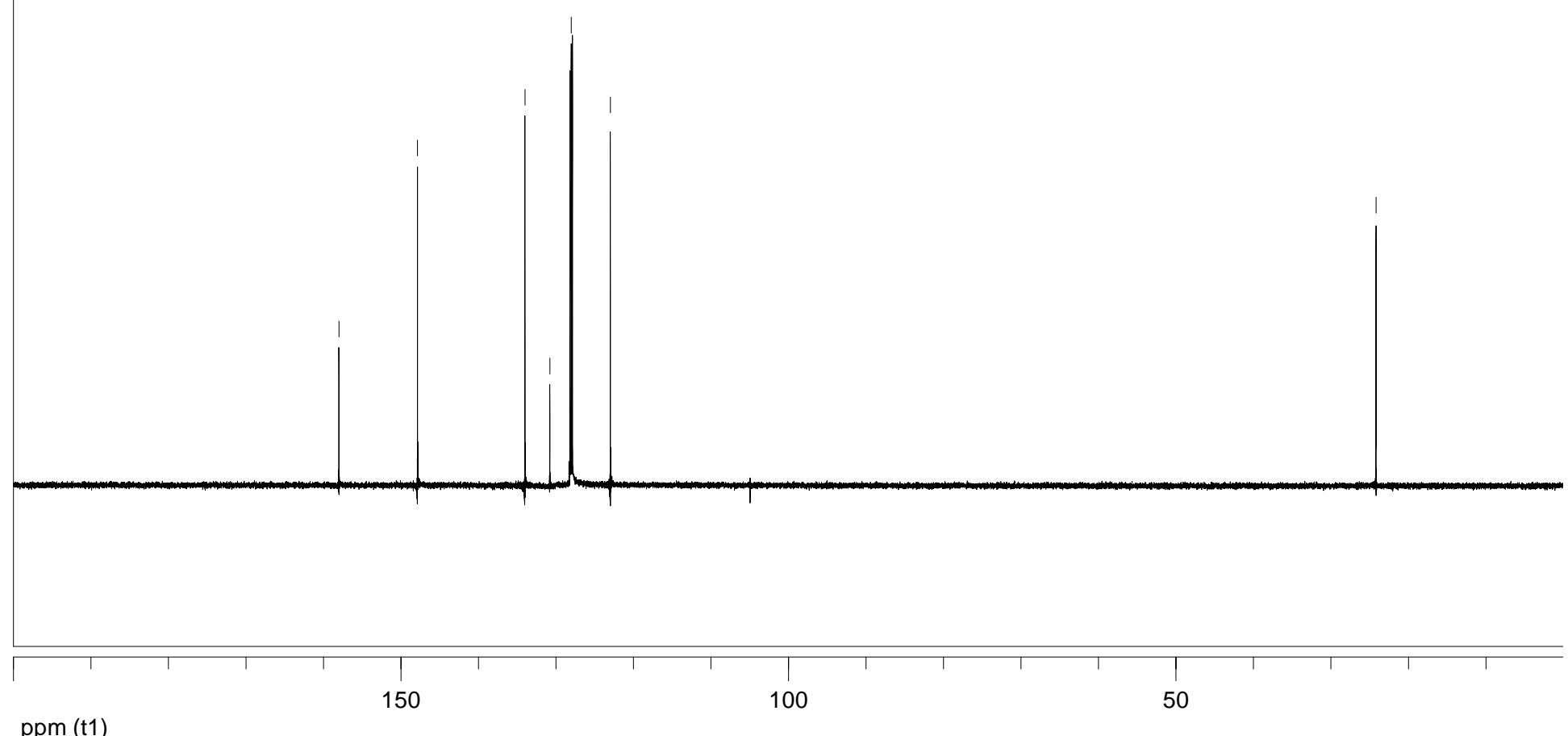


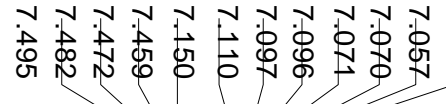

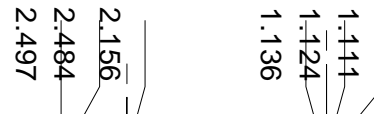

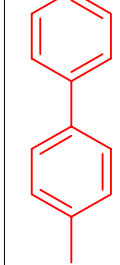

年

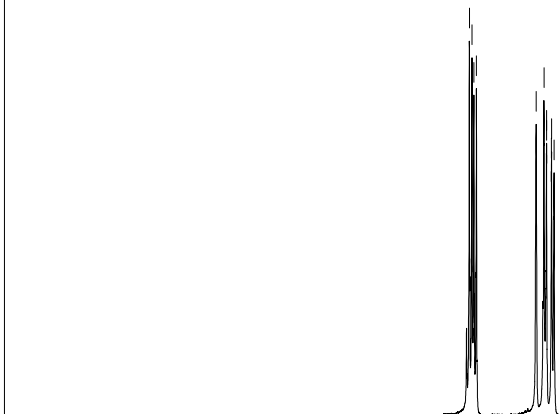




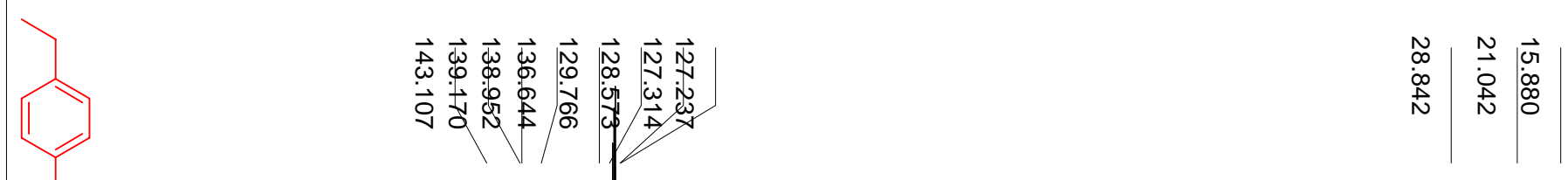

$Y$

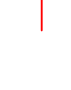
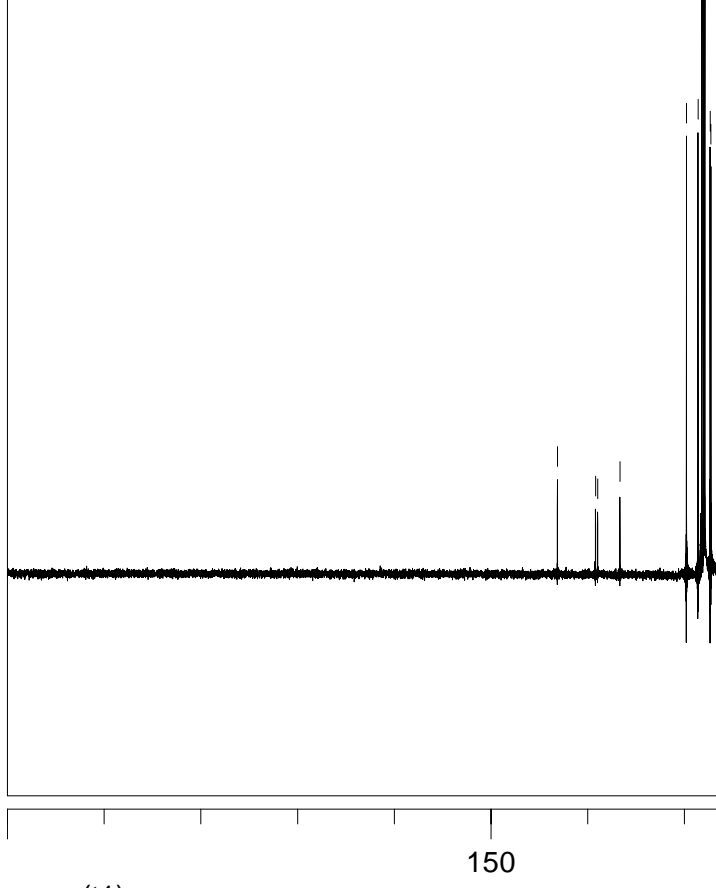

ppm (t1) 


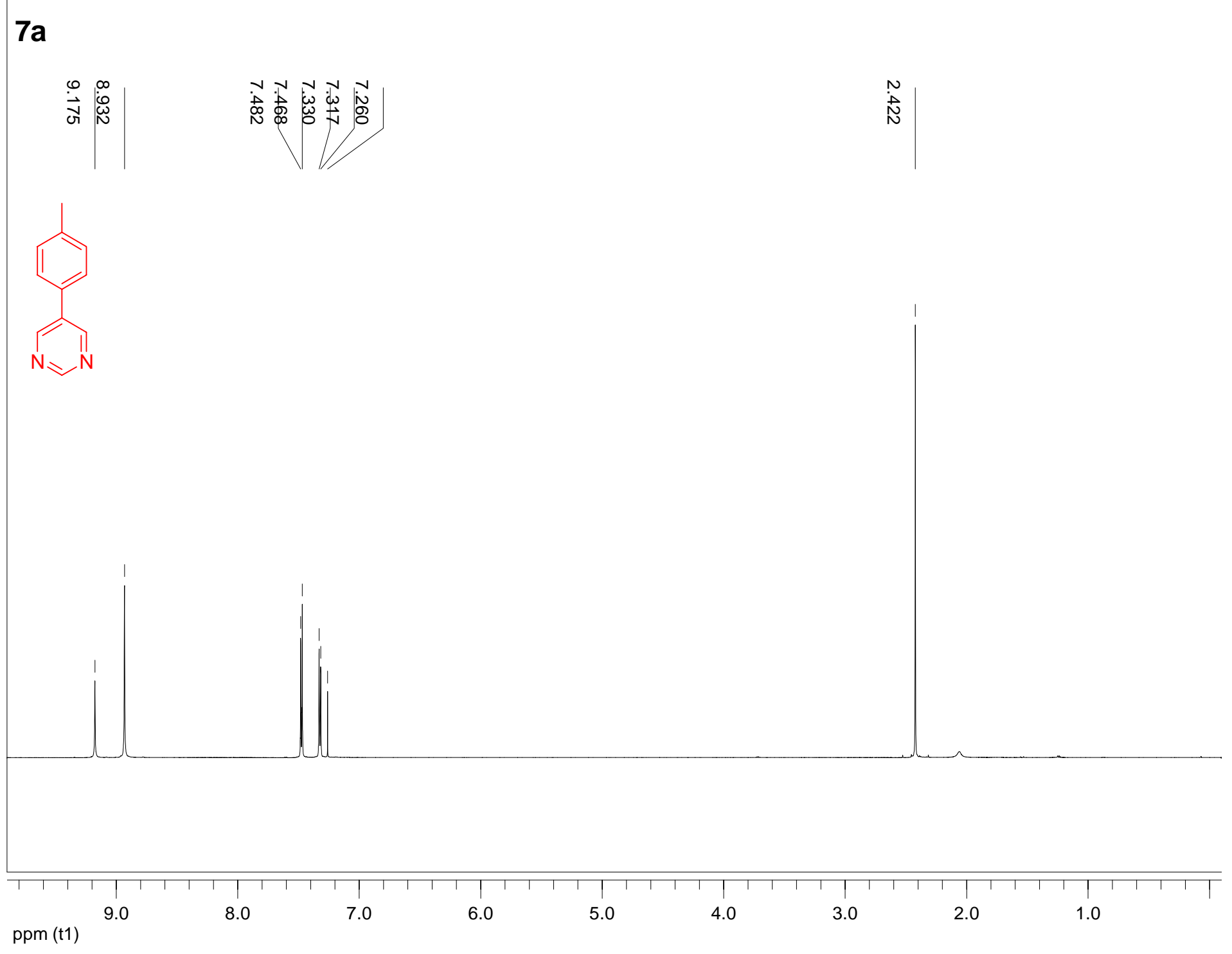




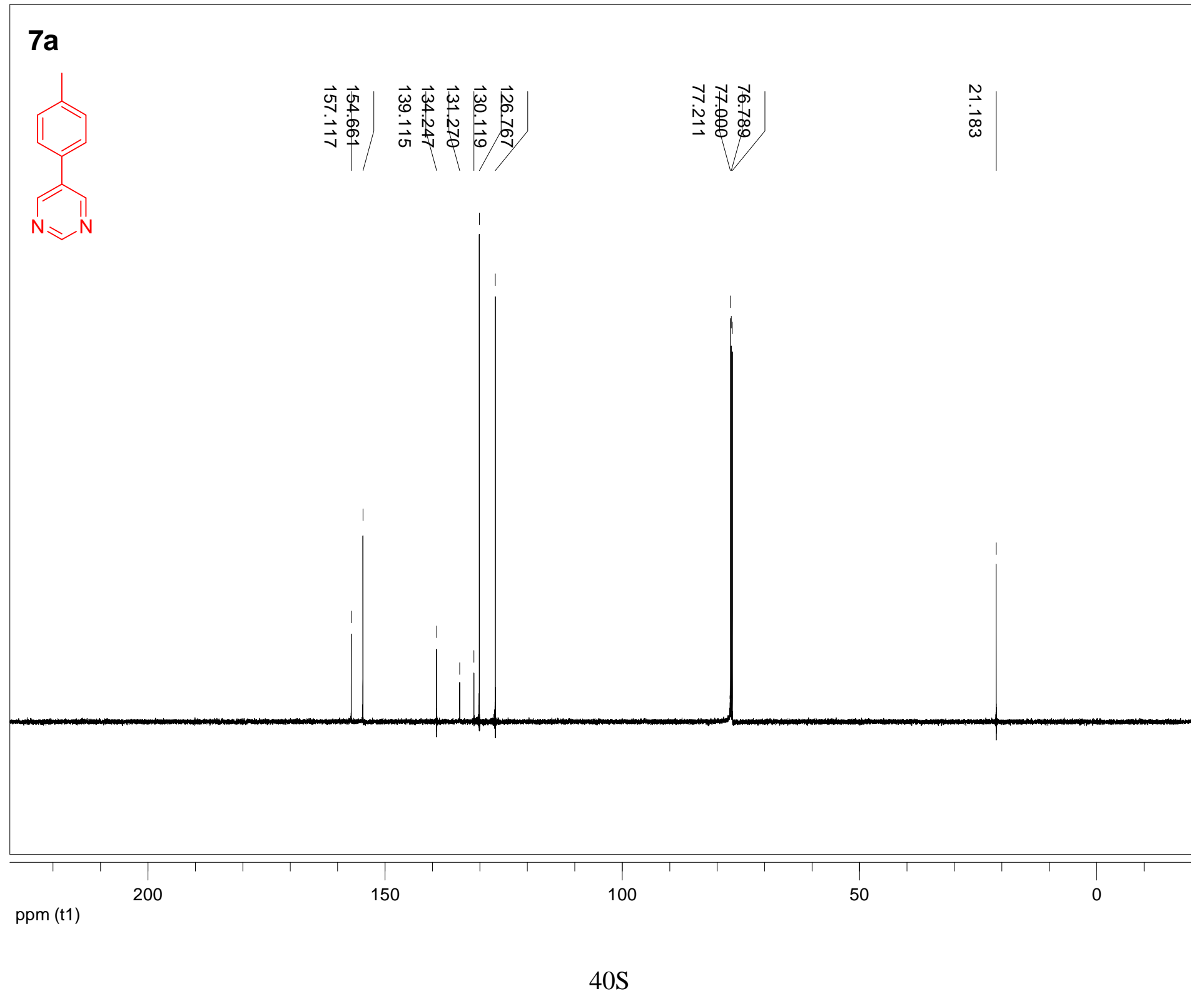




\section{$7 b$}

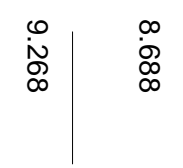

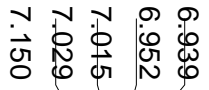

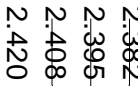
客总宫
舟
N

$\mathrm{H}_{3} \mathrm{C}$

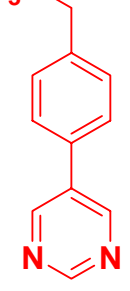

$\mathrm{N}=\mathrm{N}$
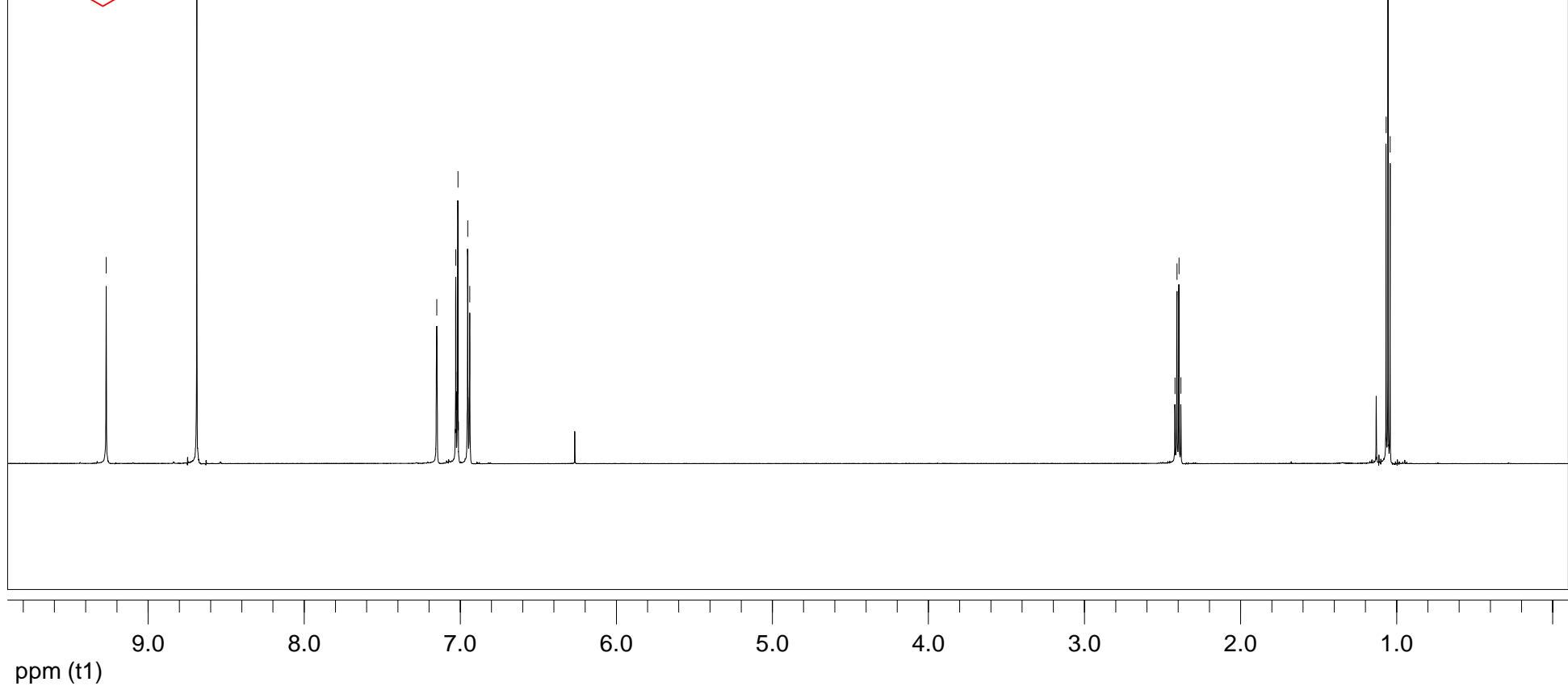


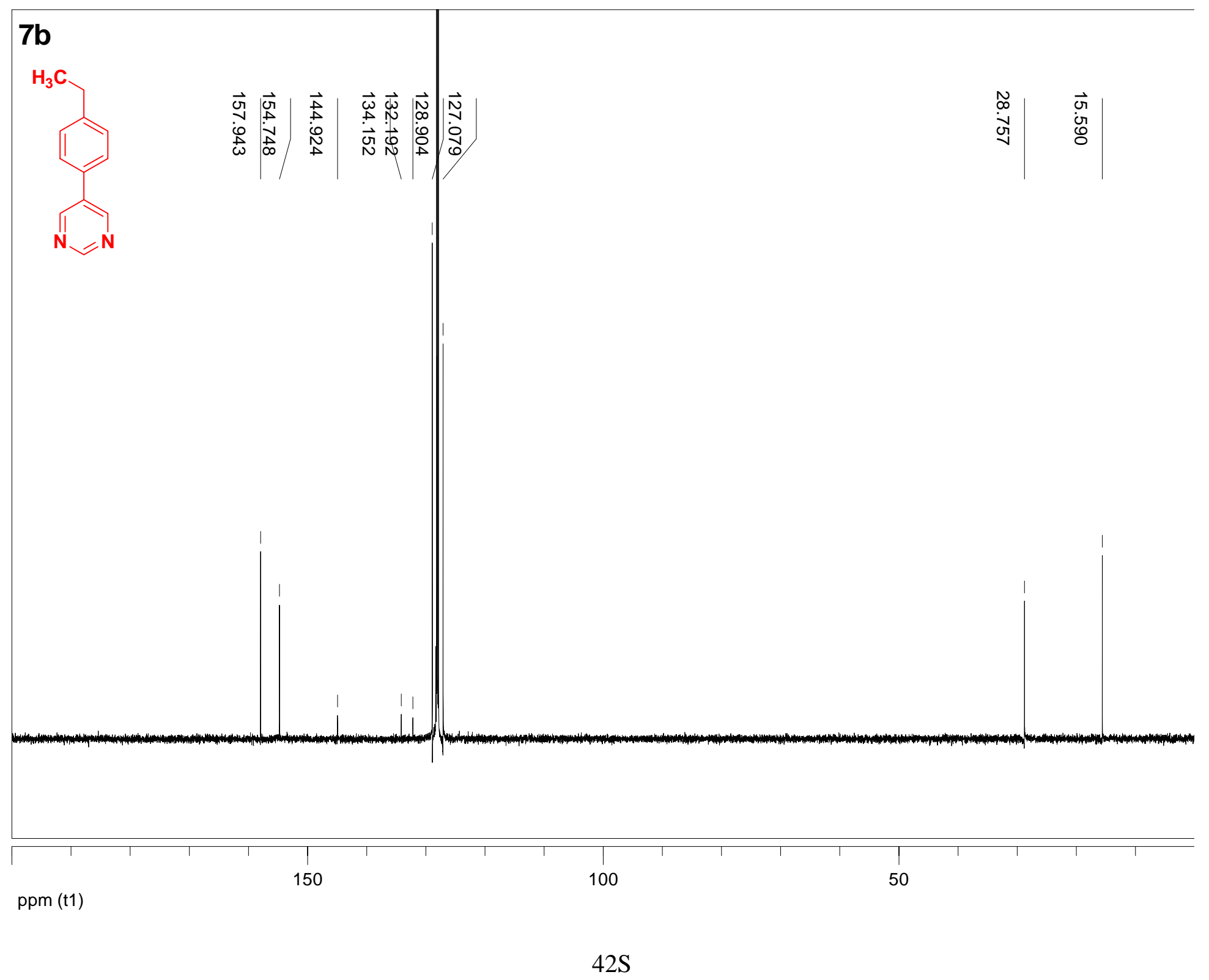


7c

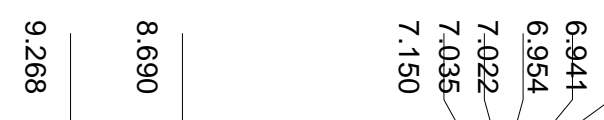

$\mathrm{CH}_{3}$

$\sum_{N=N}$

过

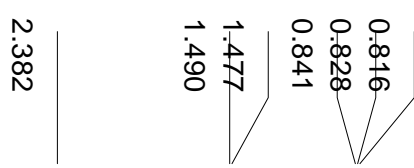

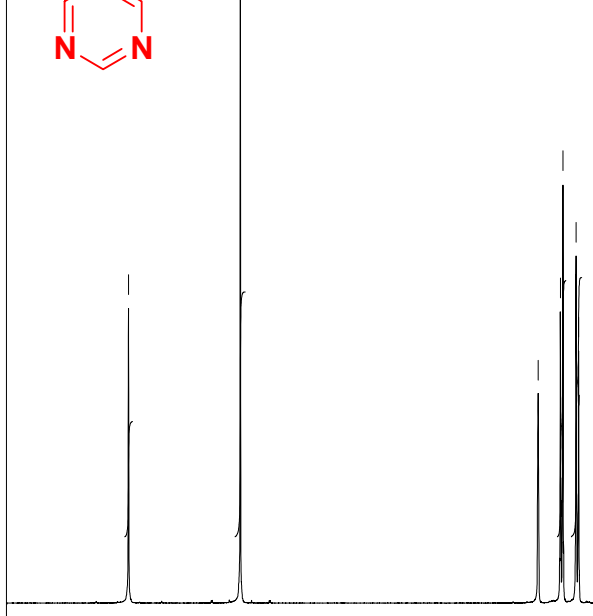

丩

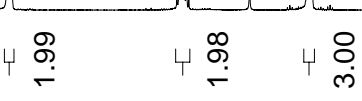

ppm (t1)

5.0 


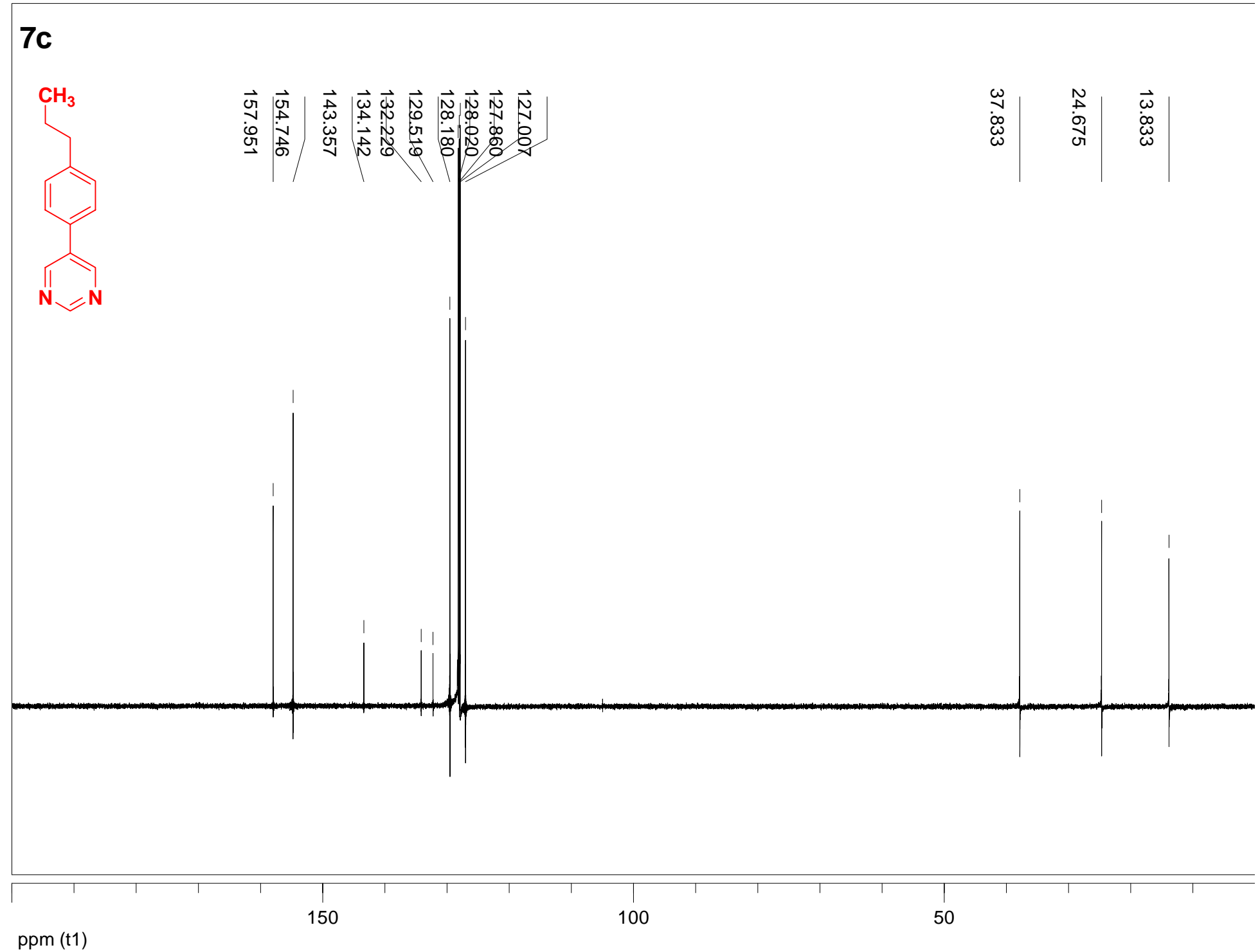



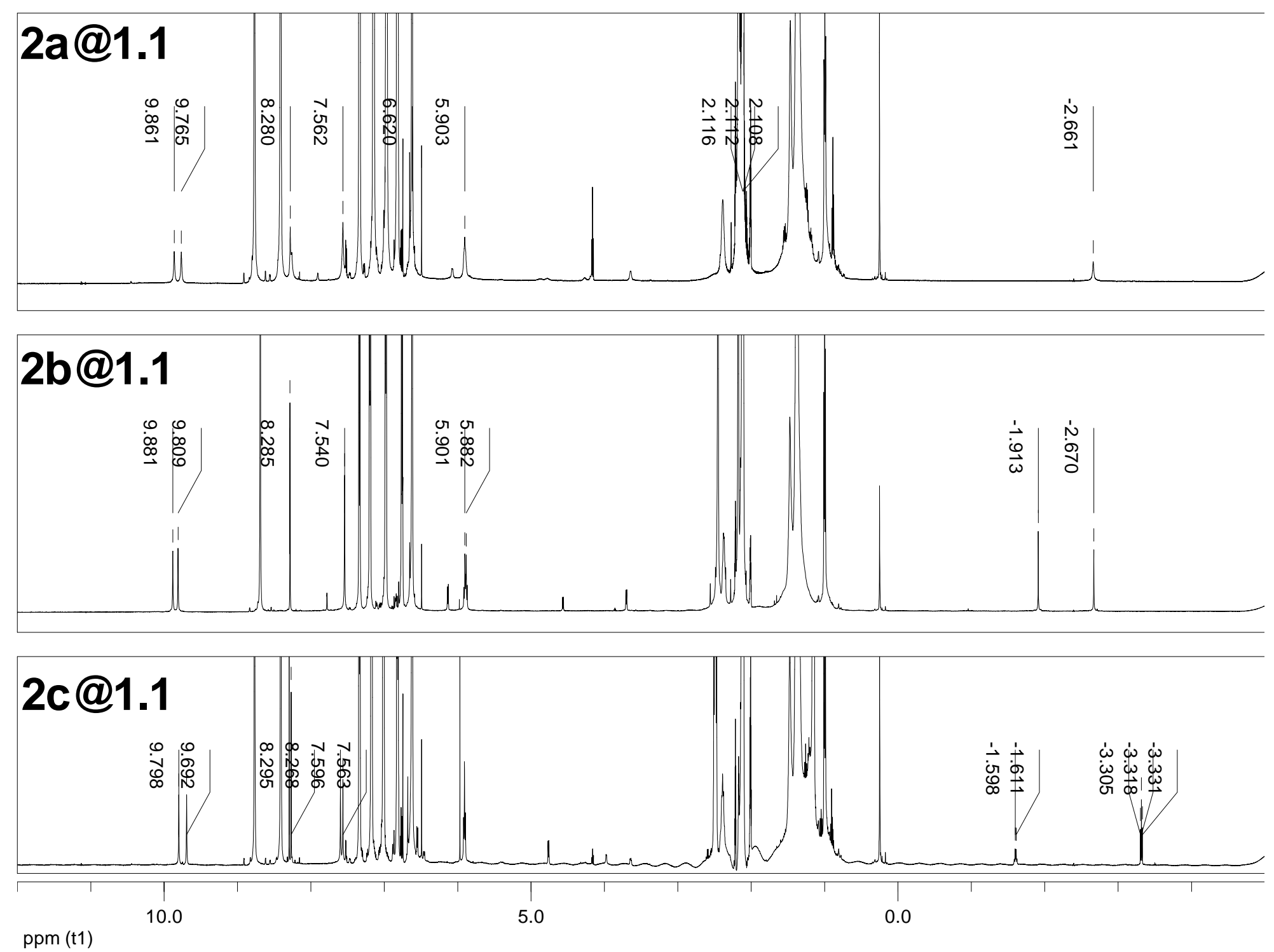

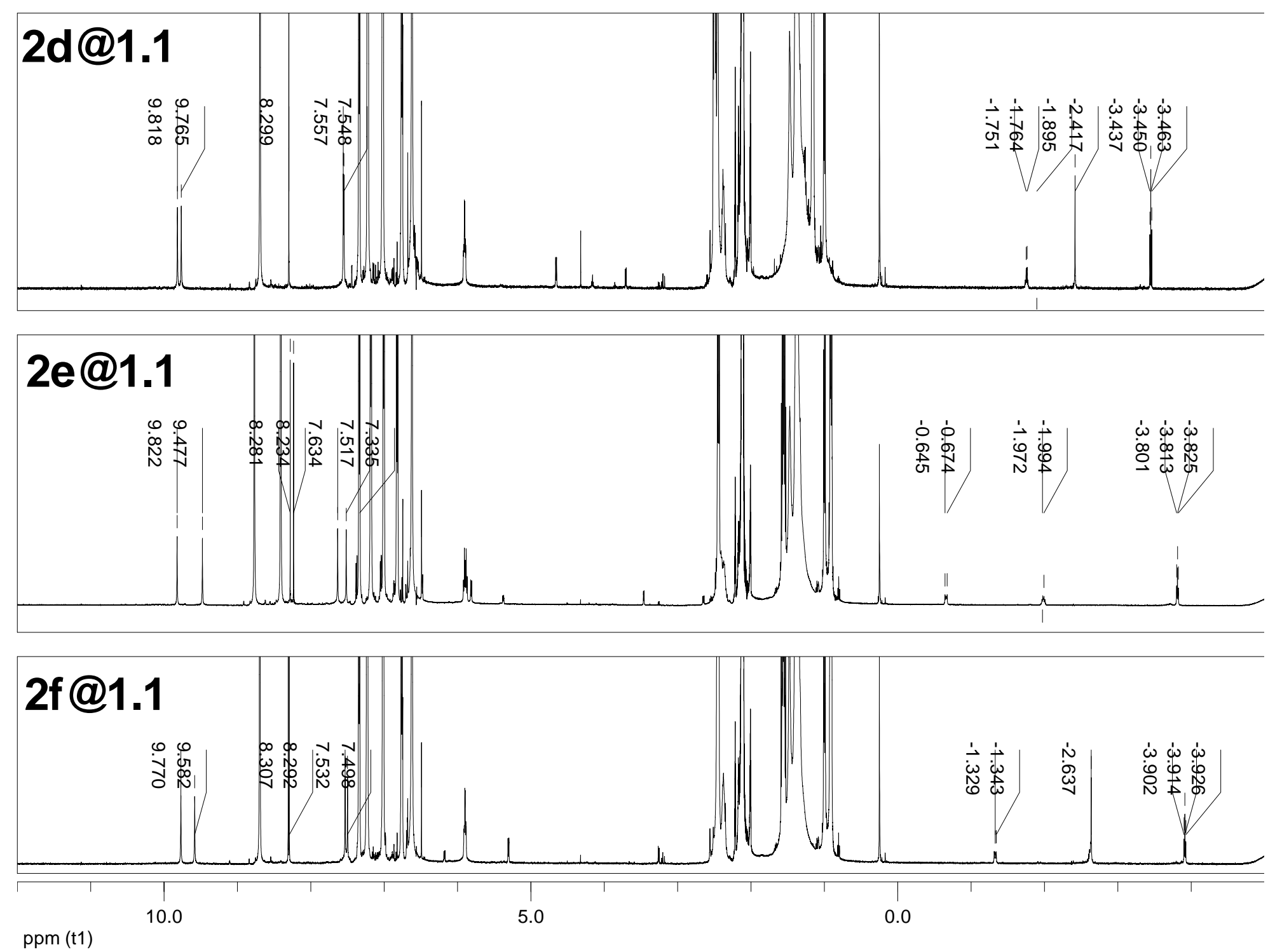

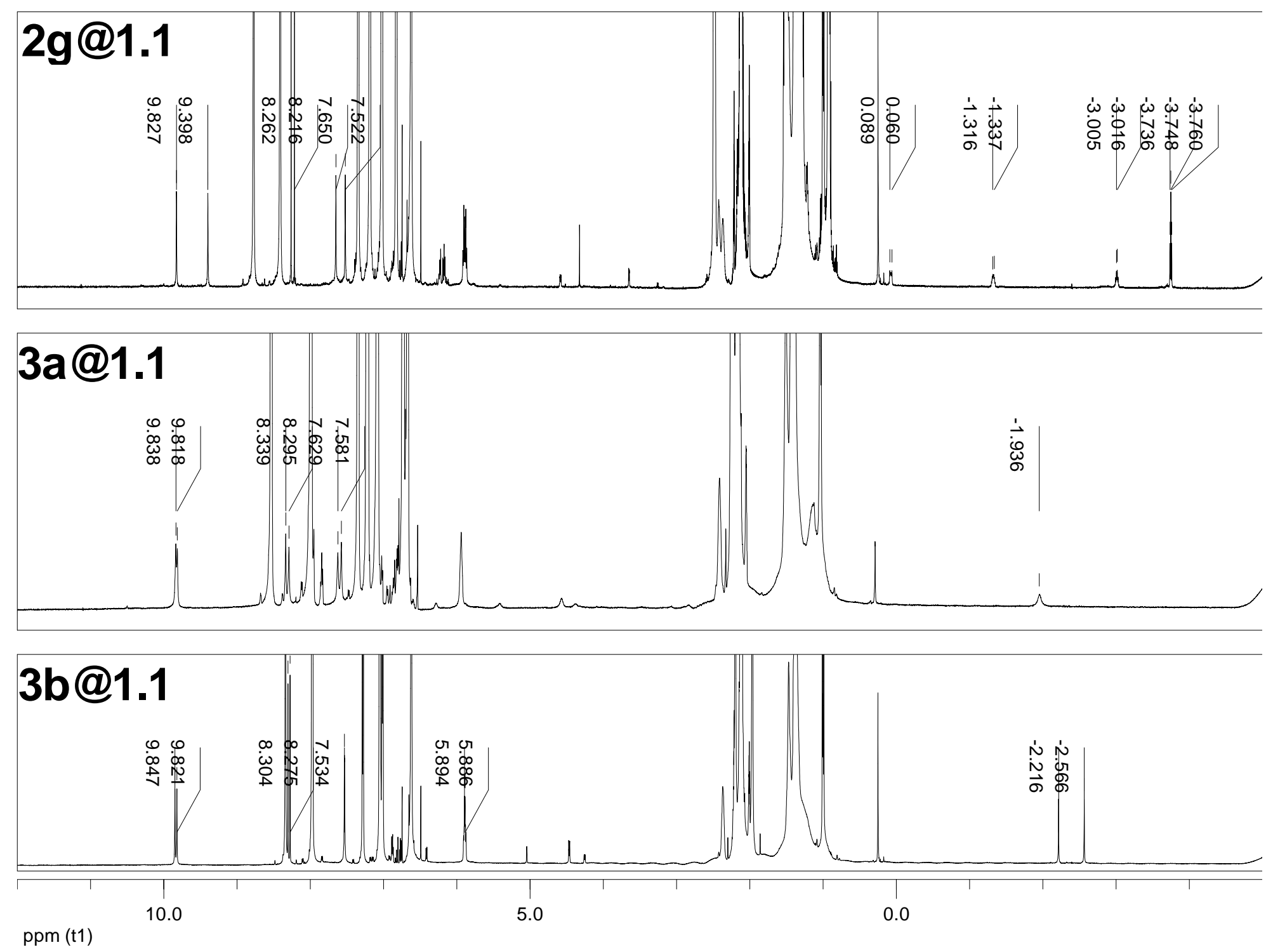

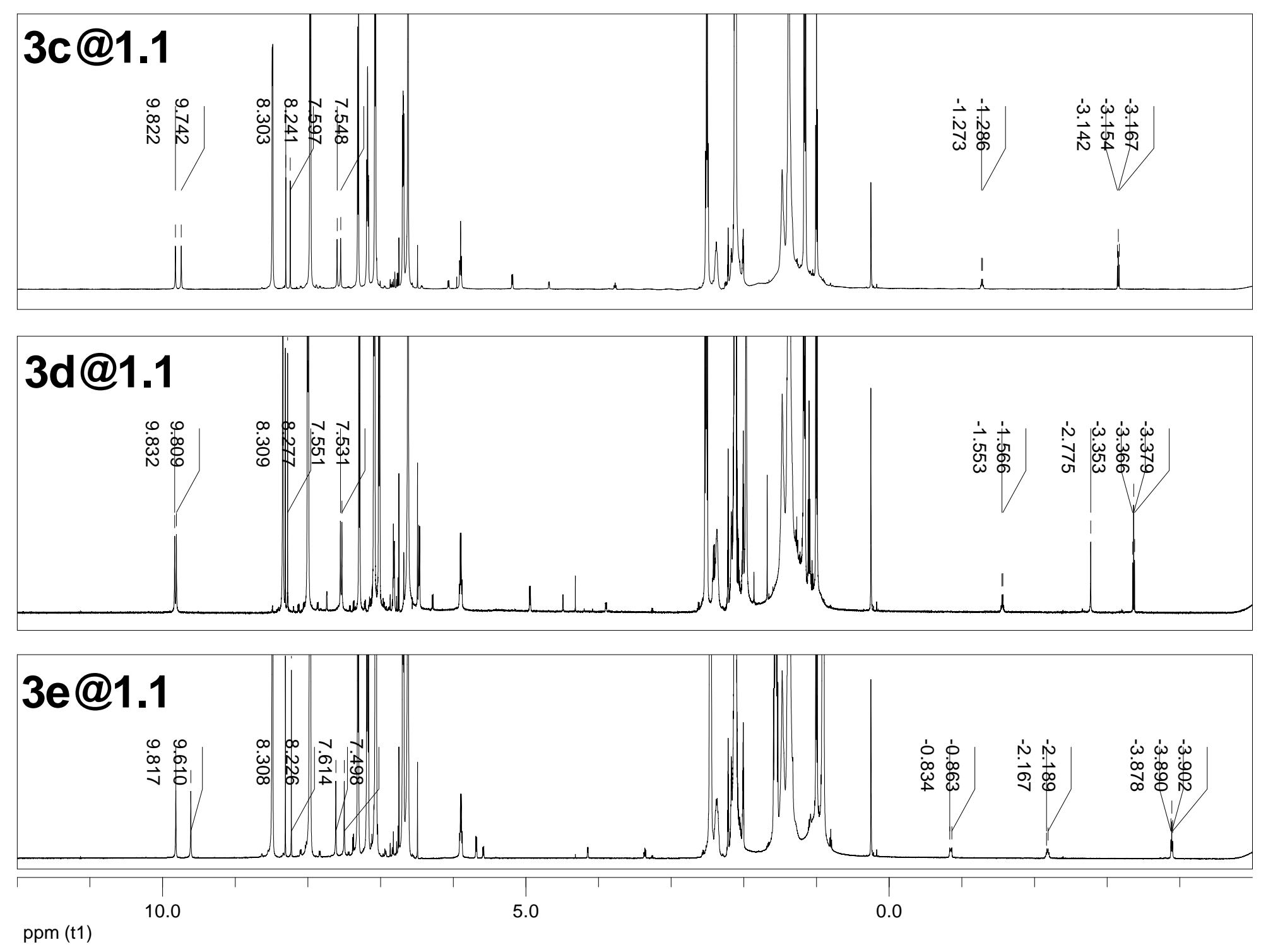

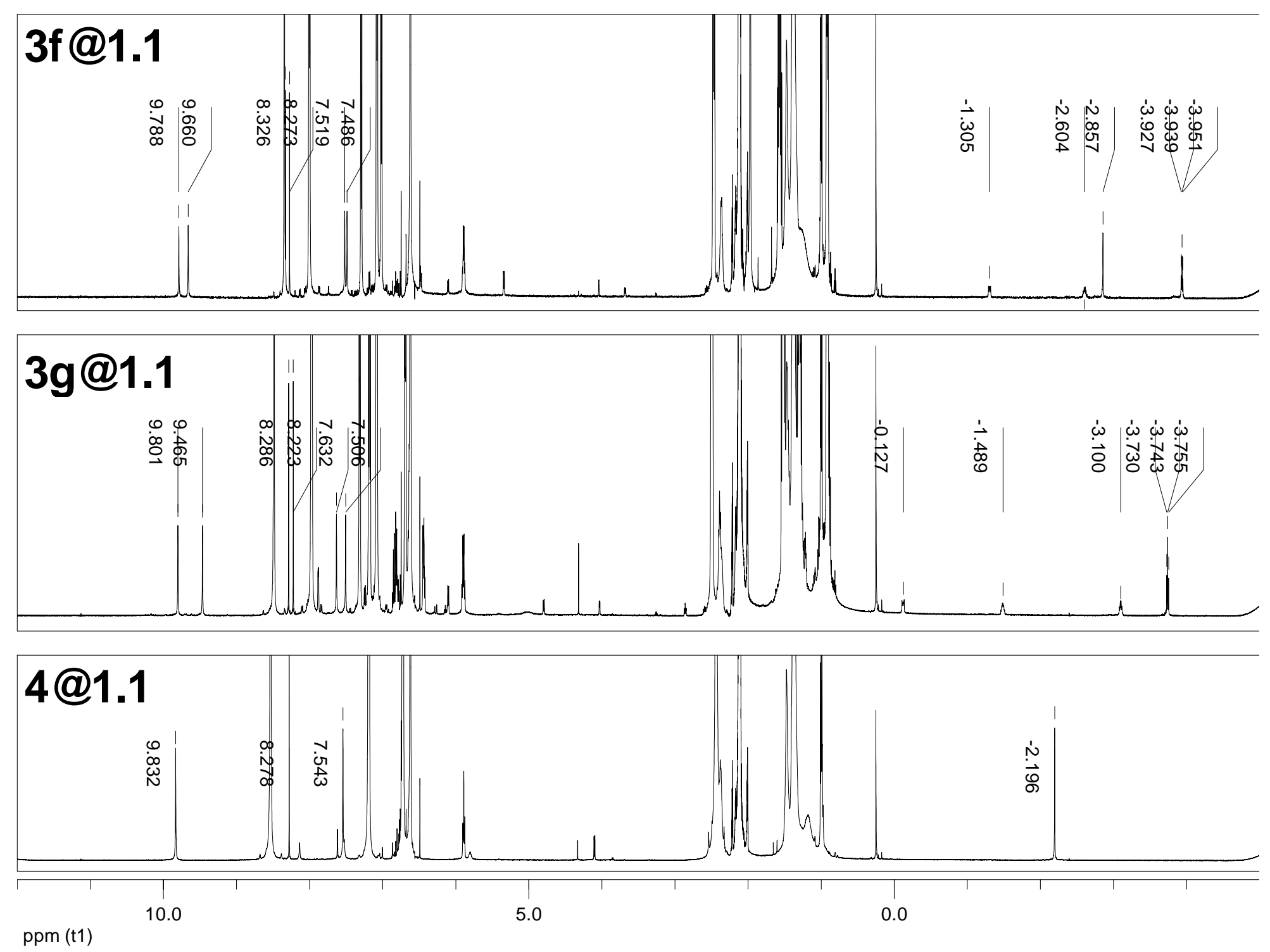

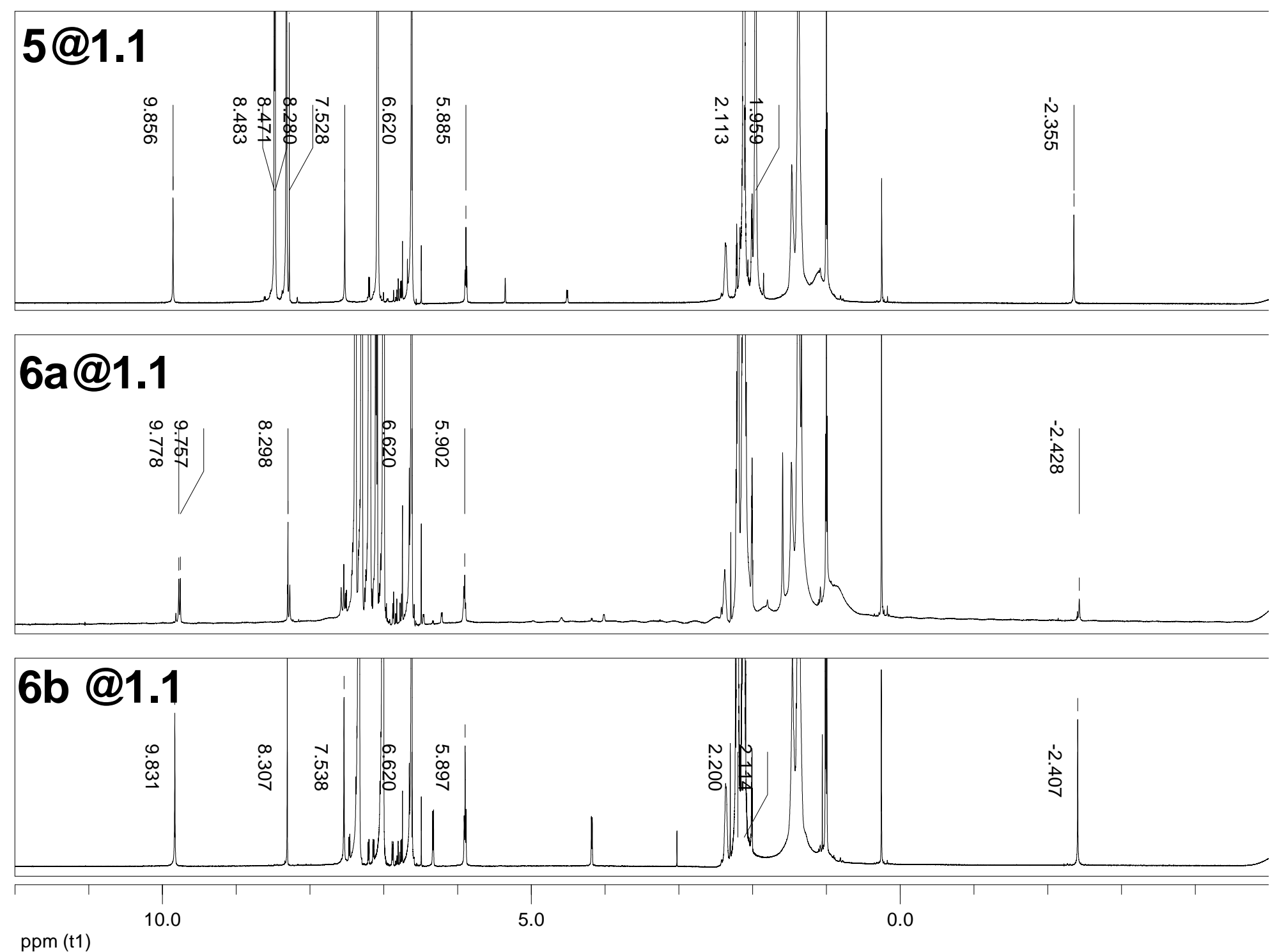

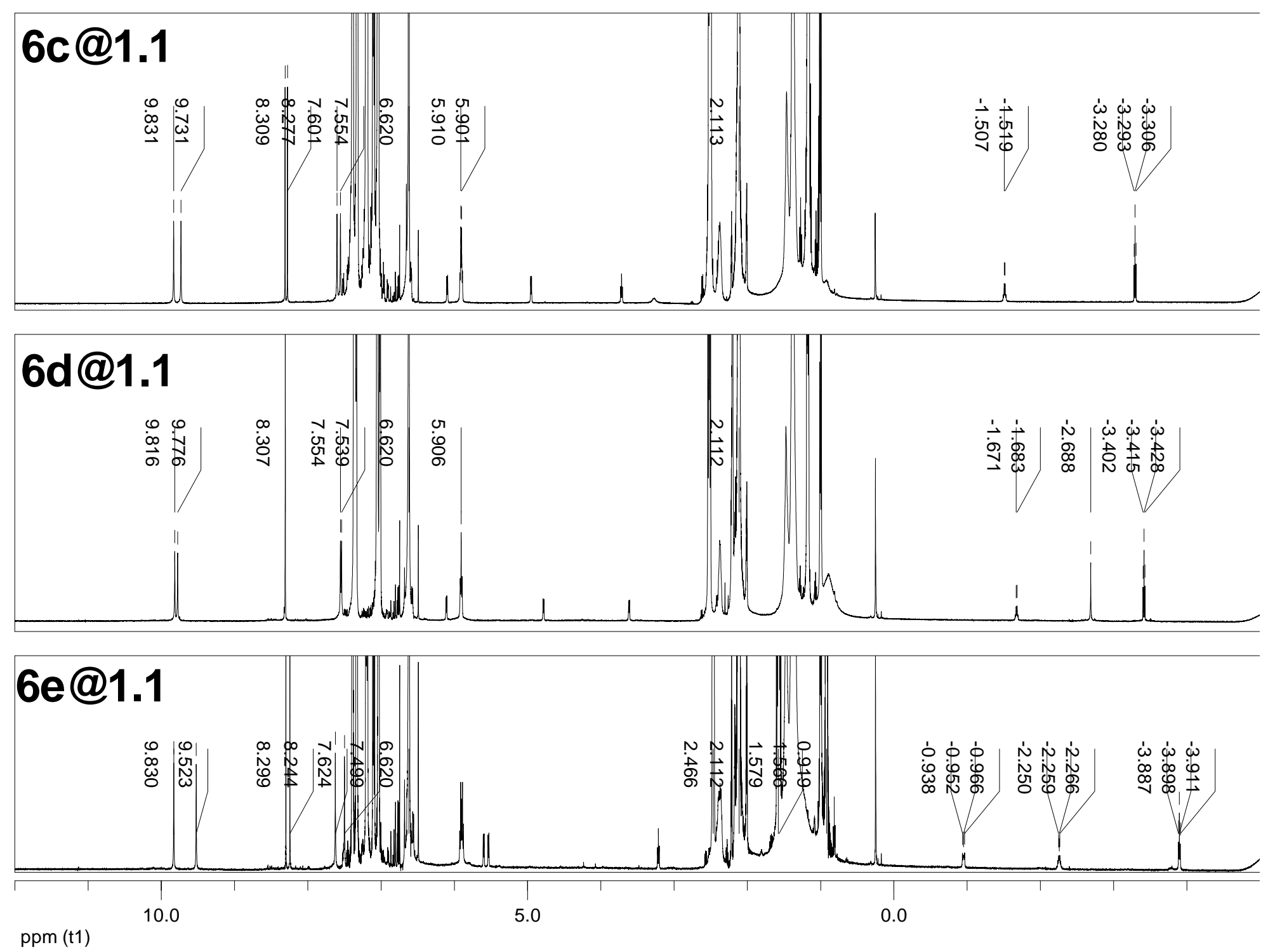

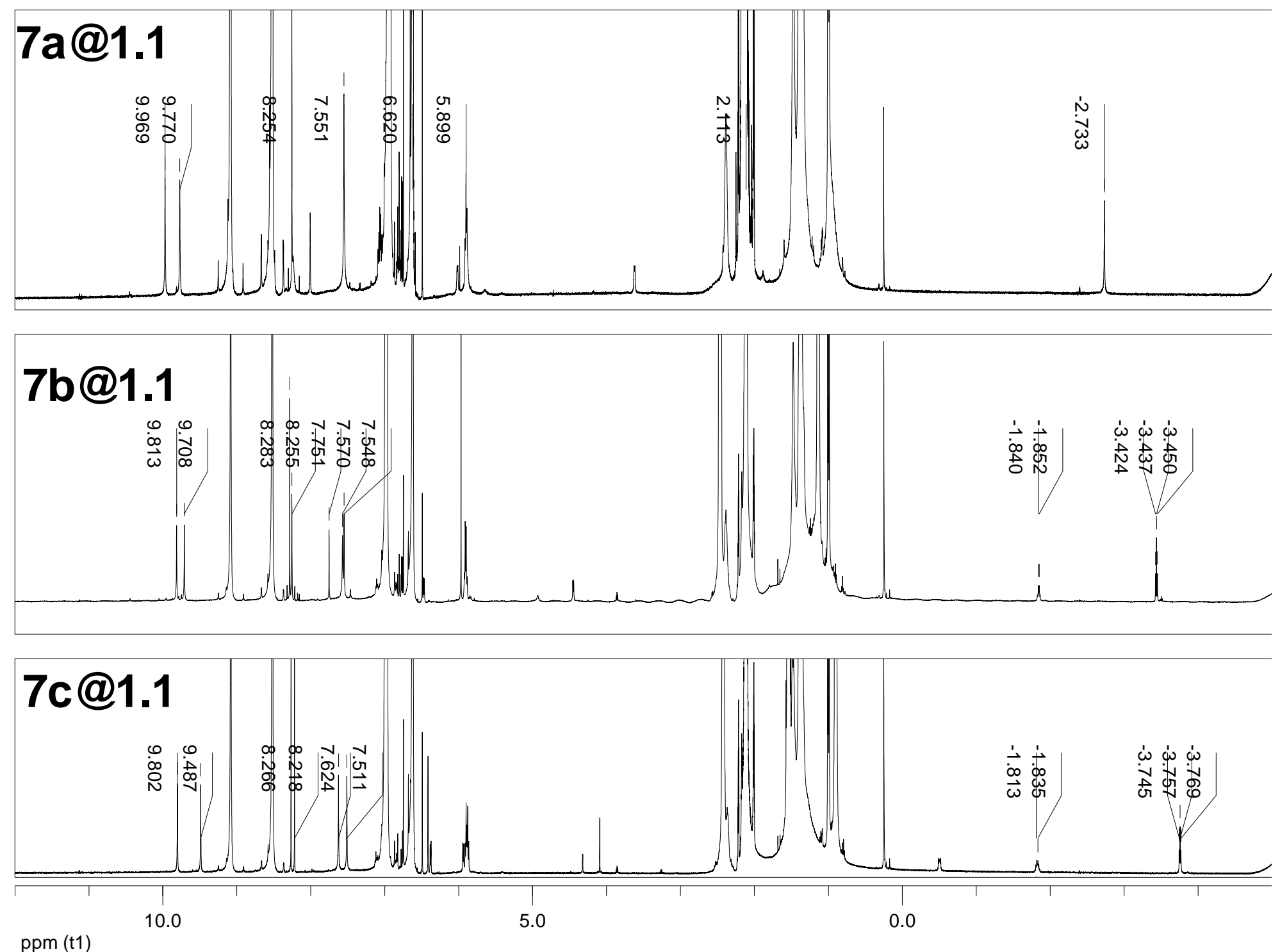


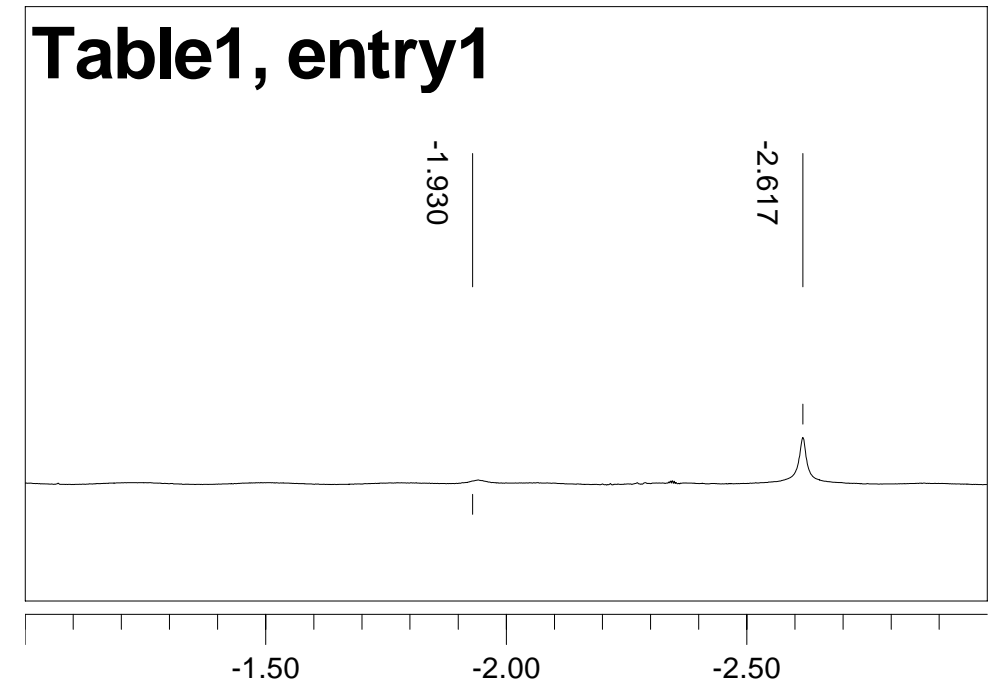

\section{Table1, entry2}

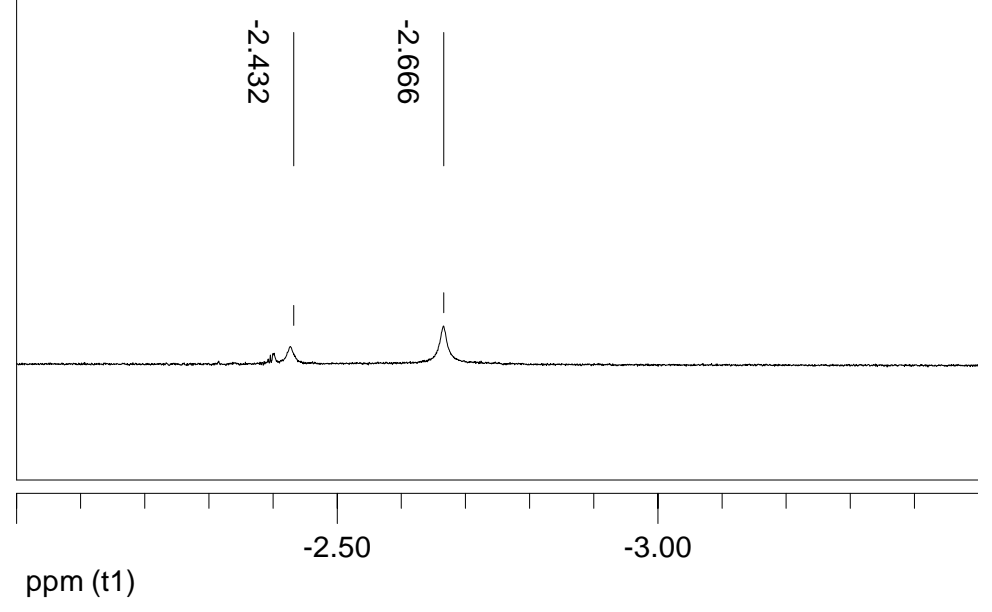

\section{Table1, entry3}

Table1, entry4

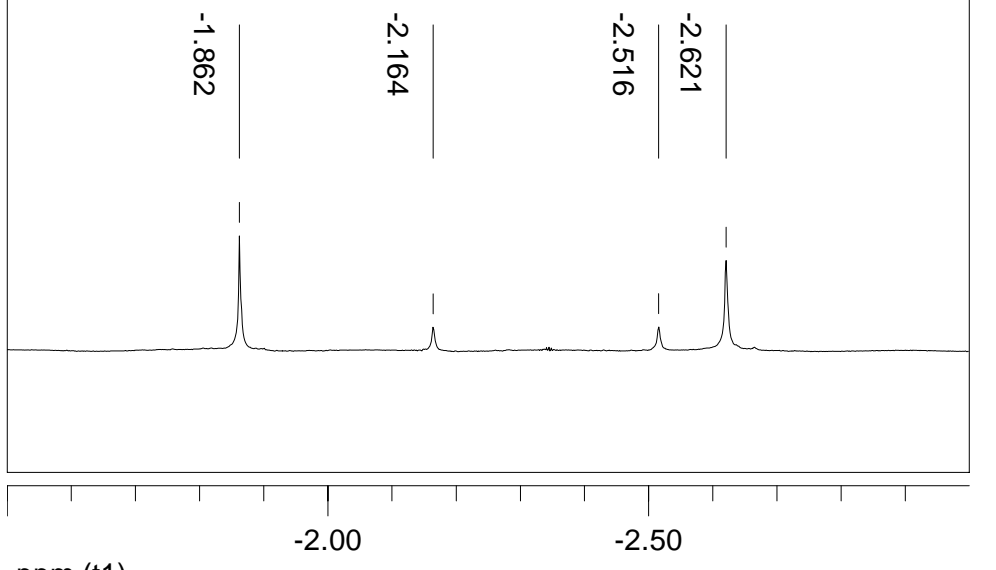

ppm (t1)

$-2.50$

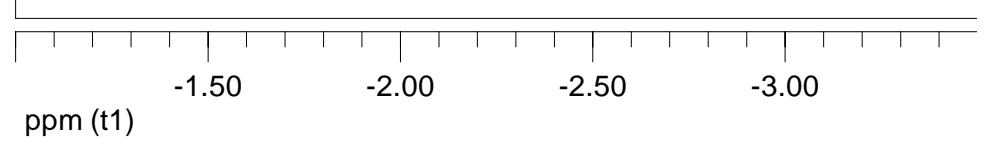




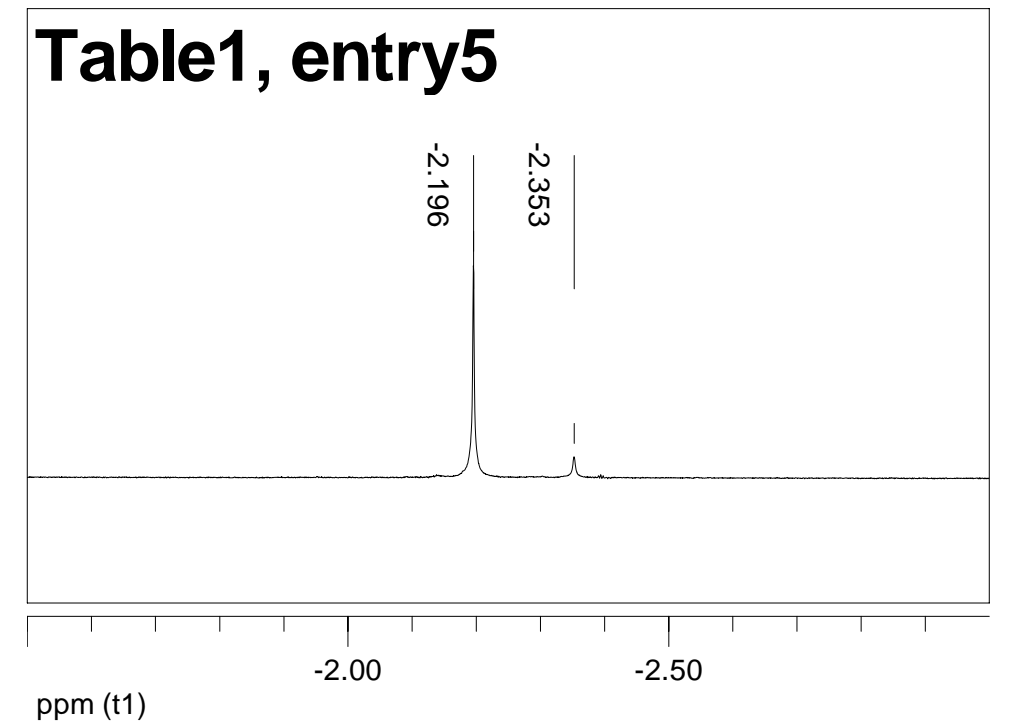

\section{Table1, entry 6}

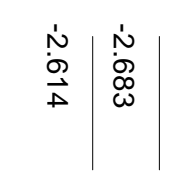

\section{Table2, entry1}

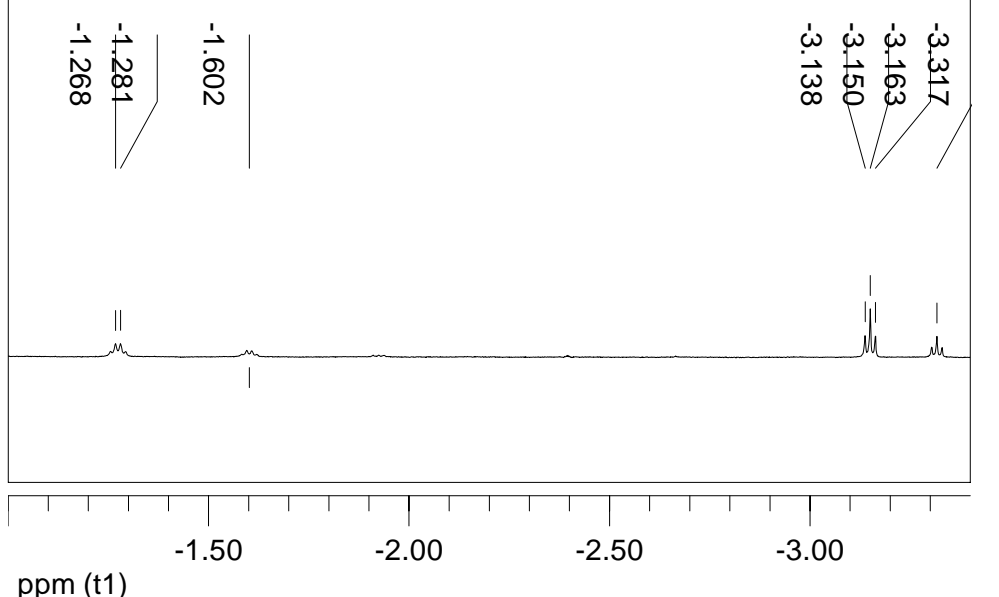

\section{Table2, entry2}

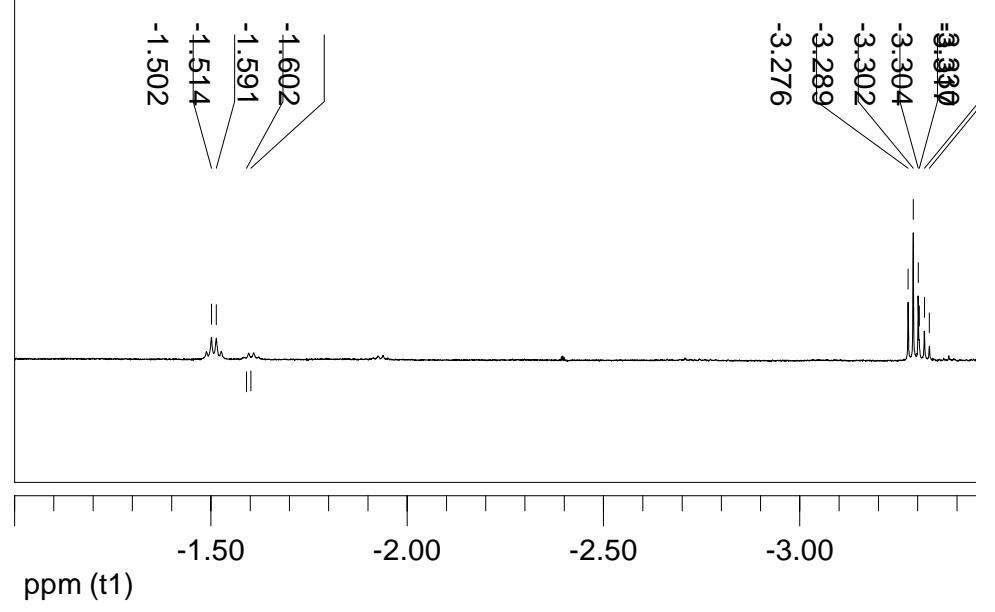




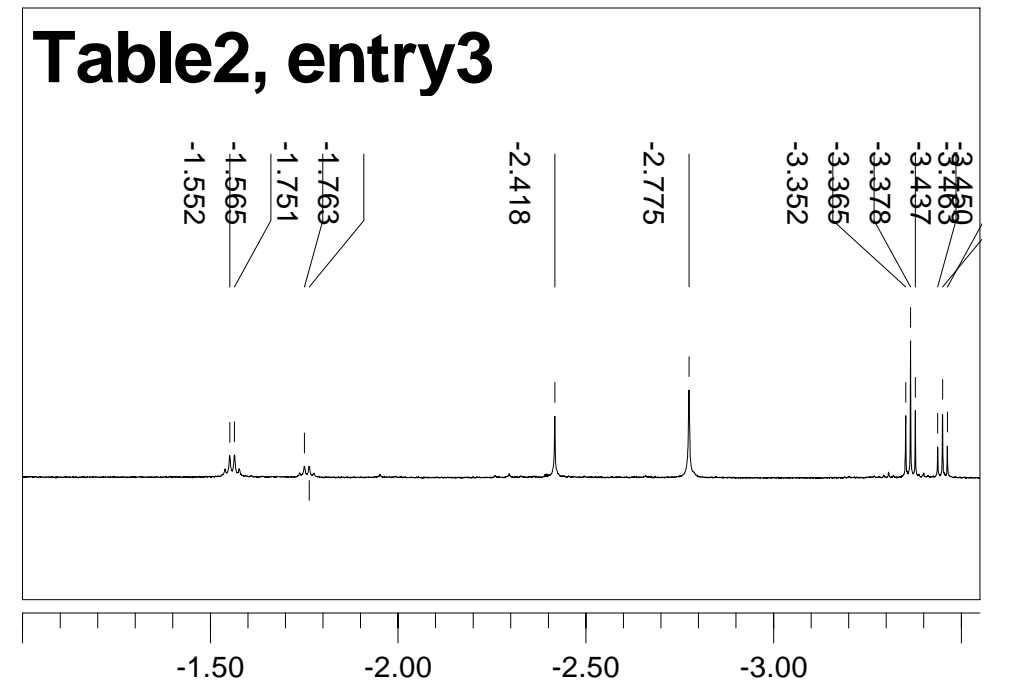

ppm (t1)

\section{Table2, entry5}

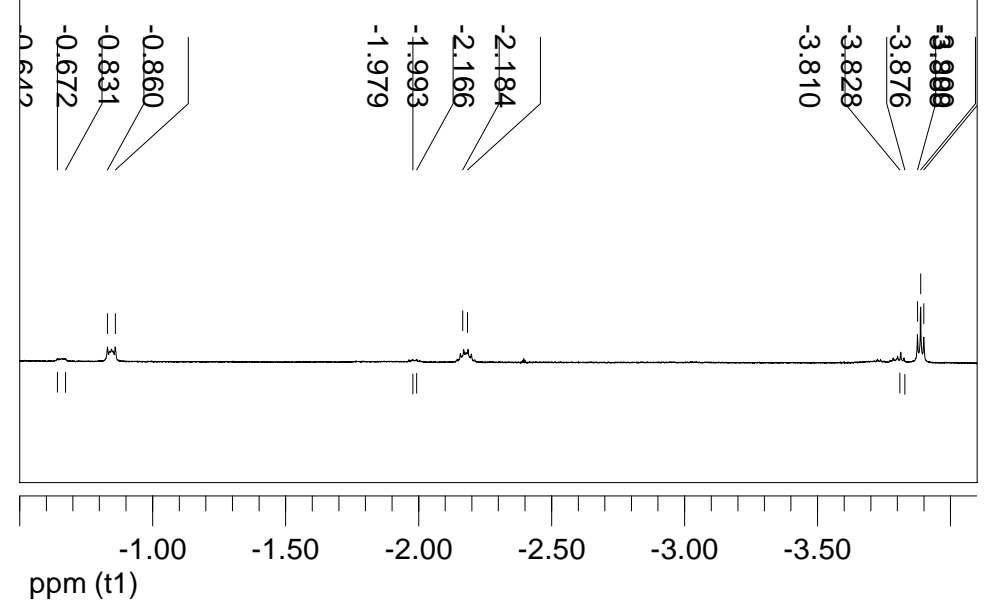

\section{Table2, entry4}

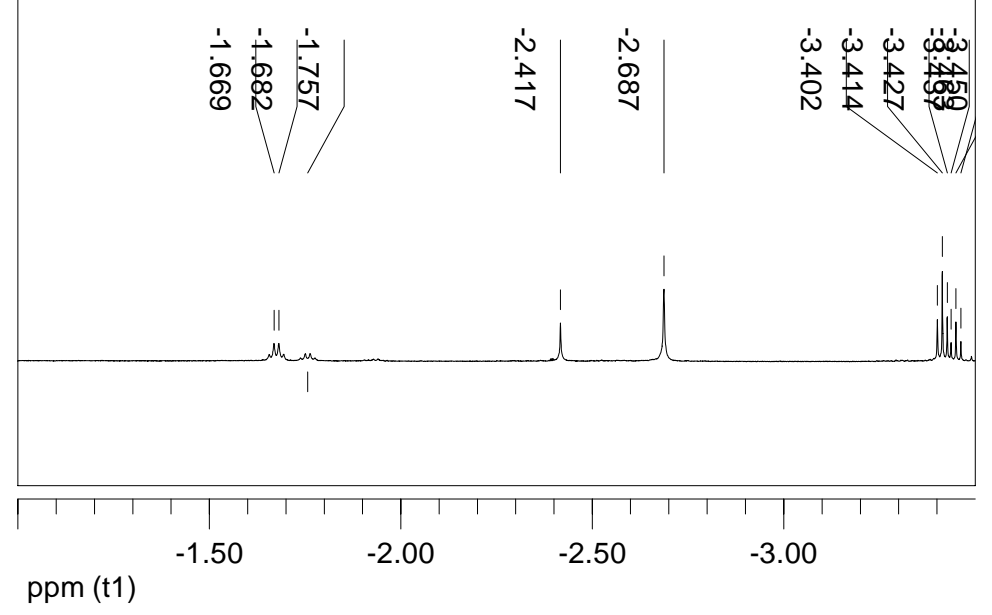

\section{Table2, entry 6}

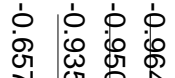
它它

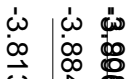

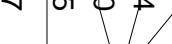

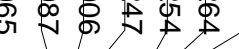
$\omega \stackrel{\infty}{\infty}$

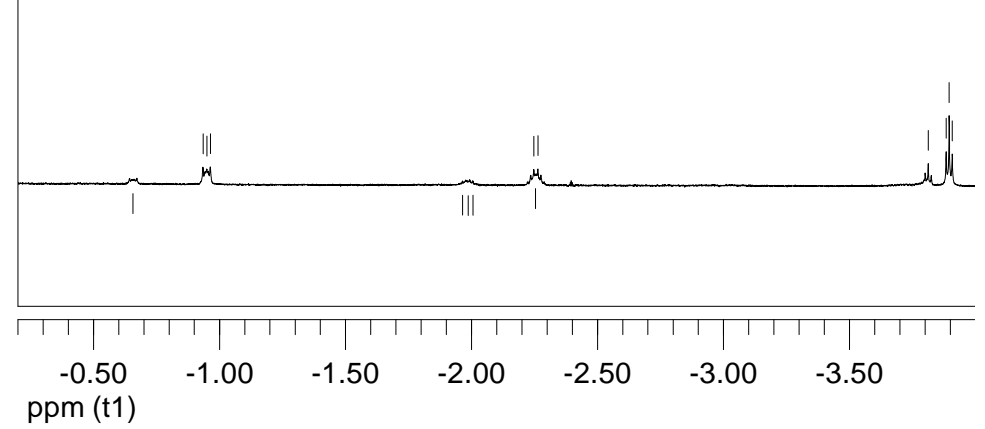




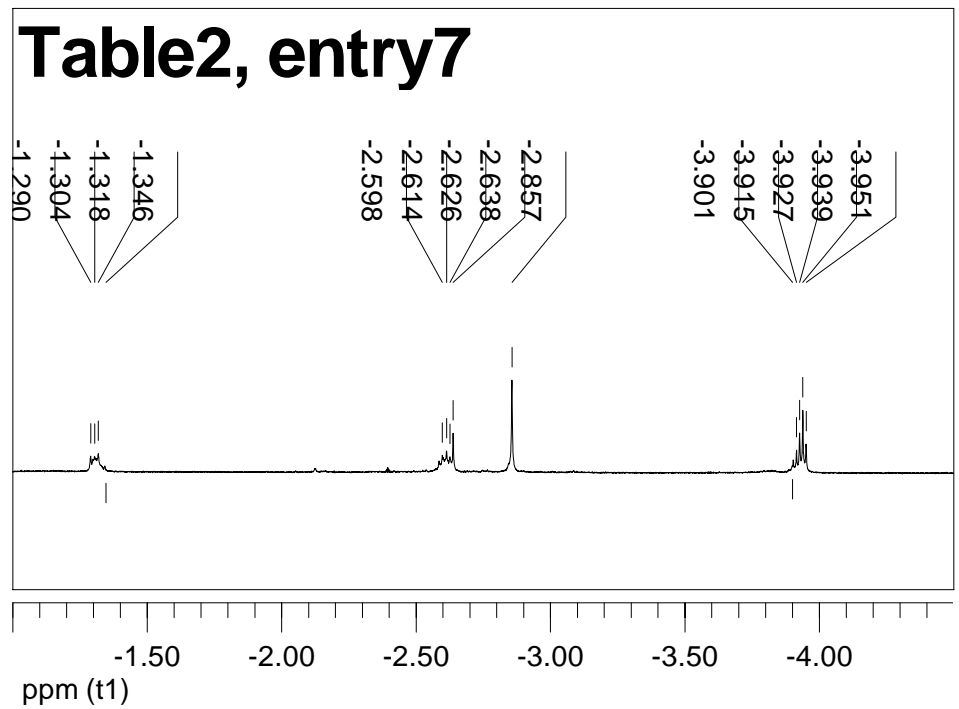

\section{Table2, entry8}

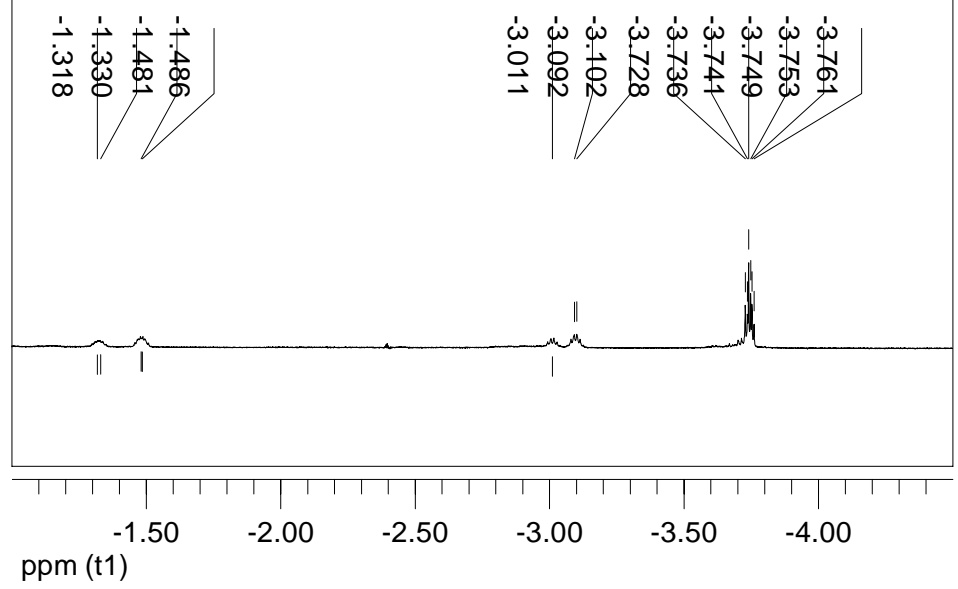

\section{Table2, entry7}

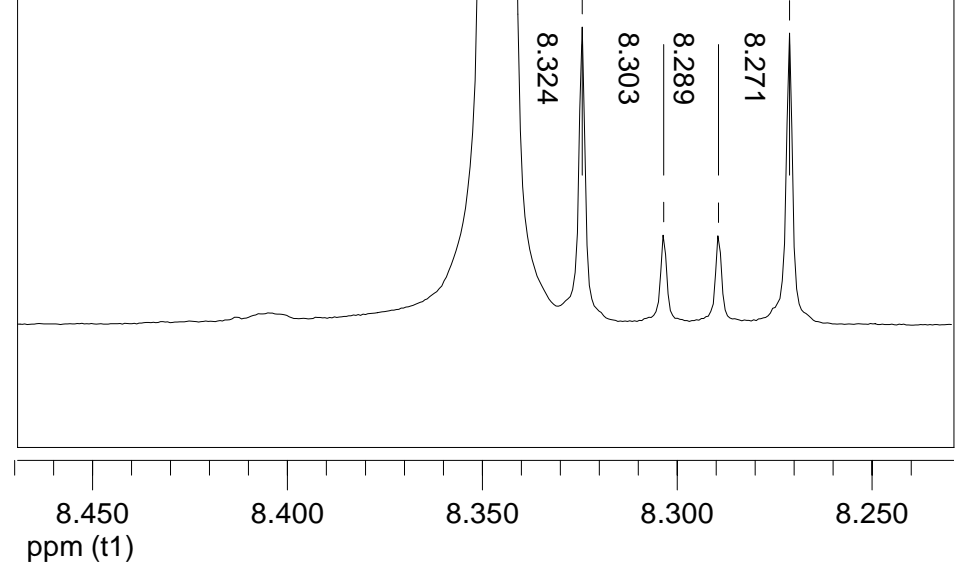

Table2, entry 9

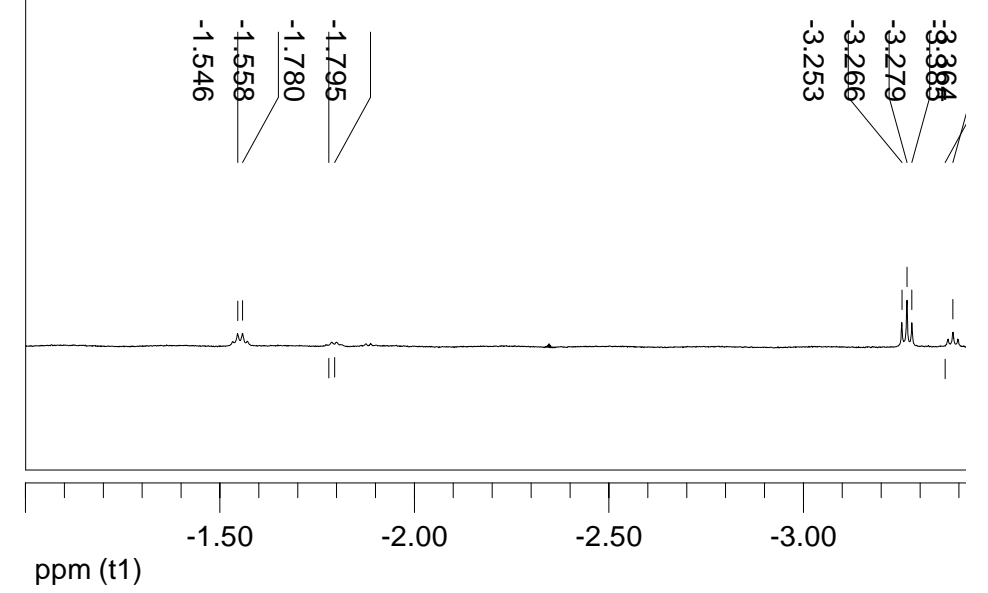




\section{Table2, entry10}

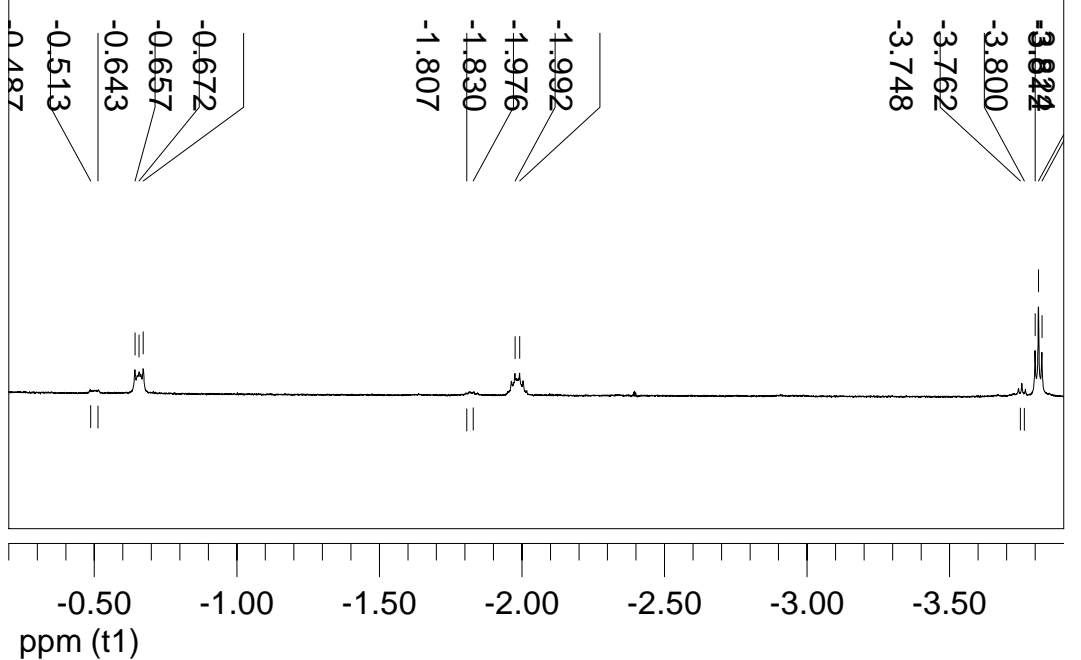

